\title{
Quantum hyperbolic geometry
}

\author{
STEPHANE BASEILHAC \\ RICCARDO BENEDETTI
}

\begin{abstract}
We construct a new family, indexed by odd integers $N \geq 1$, of $(2+1)$-dimensional quantum field theories that we call quantum hyperbolic field theories (QHFT), and we study its main structural properties. The QHFT are defined for marked $(2+1)-$ bordisms supported by compact oriented 3-manifolds $Y$ with a properly embedded framed tangle $L_{\mathcal{F}}$ and an arbitrary $\operatorname{PSL}\left(2, \mathbb{C}\right.$ )-character $\rho$ of $Y \backslash L_{\mathcal{F}}$ (covering, for example, the case of hyperbolic cone manifolds). The marking of QHFT bordisms includes a specific set of parameters for the space of pleated hyperbolic structures on punctured surfaces. Each QHFT associates in a constructive way to any triple $\left(Y, L_{\mathcal{F}}, \rho\right)$ with marked boundary components a tensor built on the matrix dilogarithms, which is holomorphic in the boundary parameters. When $N=1$ the QHFT tensors are scalar-valued, and coincide with the Cheeger-Chern-Simons invariants of $\operatorname{PSL}(2, \mathbb{C})$-characters on closed manifolds or cusped hyperbolic manifolds. We establish surgery formulas for QHFT partitions functions and describe their relations with the quantum hyperbolic invariants of Baseilhac and Benedetti [3; 4] (either defined for unframed links in closed manifolds and characters trivial at the link meridians, or cusped hyperbolic 3-manifolds). For every $\operatorname{PSL}(2, \mathbb{C})$-character of a punctured surface, we produce new families of conjugacy classes of "moderately projective" representations of the mapping class groups.
\end{abstract}

57M27, 57Q15; 57R20, 20G42

\section{Introduction}

In this paper we construct a new family $\left\{\mathcal{H}_{N}\right\}$, indexed by the odd integers $N \geq 1$, of $(2+1)$-dimensional quantum field theories (QFT) that we call Quantum Hyperbolic Field Theories (QHFT). Here, following Atiyah [2] and Turaev [32], by QFT we mean a functor from a $(2+1)$-bordism category, possibly not purely topological, to the tensorial category of finite dimensional complex linear spaces.

The QHFT bordism category is based on triples $\left(Y, L_{\mathcal{F}}, \rho\right)$, where $Y$ is a compact oriented 3-manifold, possibly with nonempty boundary $\partial Y, L_{\mathcal{F}}$ is a properly embedded framed tangle (ie a framed 1-dimensional nonoriented submanifold) in $Y$, and $\rho$ is a flat $\operatorname{sl}(2, \mathbb{C})$-connection on $Y \backslash L_{\mathcal{F}}$ up to gauge equivalence (ie a $P S L(2, \mathbb{C})$-character 
of $Y \backslash L_{\mathcal{F}}$ ), with arbitrary holonomy at the meridians of the tangle components. We require furthermore that $L_{\mathcal{F}}$ is nonempty when $N>1$, and that it intersects each of the boundary components, if any.

We will also consider a variant, denoted $\mathrm{QHFT}^{0}$, such that the tangles $L$ are unframed, while the characters $\rho$ are defined on the whole of $Y$, that is the meridian holonomies are trivial. Finally we consider a "fusion" of QHFT and QHFT" (still denoted QHFT) that incorporates both, by considering tangles having a framed part $L_{\mathcal{F}}$ as well as an unframed one $L^{0}$ (see Section 5.3).

The objects of the bordism category are suitably marked surfaces. Every such a QHFT surface is a diffeomorphism $f:(S, \mathcal{T}, p(\beta)) \rightarrow \Sigma$, where $\mathcal{T}$ is a so called "efficient triangulation" of a fixed base oriented surface $S$ with genus $g$ and $r$ marked framed points $p_{i}(r>0$ and $r>2$ if $g=0)$, and $\beta$ is any $\operatorname{PSL}(2, \mathbb{C})$-character of $S \backslash\left\{p_{i}\right\}$, represented by points $p(\beta)$ in specific parameter spaces for the representation variety $\operatorname{Hom}\left(\pi_{1}\left(S \backslash\left\{p_{i}\right\}\right), P S L(2, \mathbb{C})\right)$, built on $\mathcal{T}$ and particularly well suited for the QHFT. In fact we construct several such parameter spaces with small "residual gauge groups" acting on them, and we point out the relations to each other. One of them, the so called (-)-exponential $\mathcal{I}$-parameter space, is defined in terms of cross-ratios and incorporates the Bonahon-Thurston shear-bend coordinates for pleated hyperbolic surfaces with punctures.

Every QHFT bordism has marked boundary and is considered as a "transition" from its input QHFT surfaces towards the output ones. We understand that the characters $\rho$ and $\beta$ are compatible. Every QHFT functor associates to such a transition a tensor called the amplitude, defined up to a sign and multiplication by $N$-th roots of unity. When $N=1$, the QHFT functor is obtained by extending to QHFT bordisms the simplicial formulas of Neumann [24] of the Cheeger-Chern-Simons invariants of PSL (2, C)characters on closed manifolds, or finite volume noncompact complete hyperbolic 3-manifolds (ie cusped manifolds). In particular, this functor is scalar-valued.

When $Y=W$ is closed (that is $\partial Y=\varnothing$ ), for any $N$ the amplitudes $\mathcal{H}_{N}\left(W, L_{\mathcal{F}}, \rho\right)$ are numerical invariants, called partition functions. When $N>1$ the $\mathrm{QHFT}^{0}$ partition functions $H_{N}(W, L, \rho)$ coincide with the "quantum hyperbolic invariants" constructed by the authors in [3; 4], while the QHFT ones yield new wide families of invariants, covering interesting geometric situations such as compact hyperbolic cone manifolds. We will analyze the relations between $\mathcal{H}_{N}$ and $H_{N}$ partition functions. When $N=1$, we know a geometric interpretation of the invariants $\mathcal{H}_{1}\left(W, L_{\mathcal{F}}, \rho\right)$ only when $\rho$ extends to the whole of $W$ : by using Theorem 6.7 of the present paper we can reach the setup of [24] (see also [4]) and, as mentioned above, identify $\mathcal{H}_{1}\left(W, L_{\mathcal{F}}, \rho\right)$ with the Cheeger-Chern-Simons invariant of the pair $(W, \rho)$, the link being immaterial. If 
$\rho$ does not extend, we get a relative version, the meaning of which deserves careful attention. For general QHFT bordisms the geometric interpretation of amplitudes for $N=1$ is even more unclear.

In [4] we defined also quantum hyperbolic invariants $H_{N}(M)$ for cusped manifolds $M$. Although these invariants are not immediately QHFT partition functions, we will show how they can be obtained in terms of these last. For that we establish a "surgery formula" for quantum hyperbolic invariants of cusped manifolds and QHFT partition functions that generalizes the one for Cheeger-Chern-Simons classes, and makes crucial use of some of W Neumann's arguments in [24, Sections 11 and 14].

By restricting QHFT to the trivial bordisms (the cylinders) we get a new family of conjugacy classes of "moderately projective" representations of the mapping class groups of punctured surfaces, that is, defined up to a sign and multiplication by $N$-th roots of unity.

We stress that we need that any bordism includes a nonempty link, intersecting each boundary component (so that the QHFT surfaces have punctures), in order to build a consistent functor when $N>1$ [4, Lemma 6.4]. Even when the holonomy is trivial around the punctures, we cannot forget them, in particular for what concerns the mapping class groups.

We show that QHFT are in fact restrictions to a geometric bordism category of "universal functors" called Quantum Hyperbolic Geometry (QHG). QHG includes the definition of a specific category of triangulated 3-dimensional pseudomanifolds equipped with additional structures, and modeled on the functional properties of the matrix dilogarithms studied in [4]. The QHG functors associate determined tensors to every such a decorated triangulation, obtained by tracing the matrix dilogarithms supported by each tetrahedron. The key point is that such tensors are invariant up to QHG triangulated pseudomanifold isomorphism. The main step in order to construct specializations with a strong geometric content, such as QHFT, consists in converting each QHFT (marked) bordism to a QHG triangulated pseudomanifold, unique up to QHG triangulated pseudomanifold isomorphism.

Hence we view this paper as a kind of achievement of the foundation of the theory initiated in $[3 ; 4]$.

A main interest in the QHFT comes from the fact that they relate classical 3-dimensional hyperbolic geometry to the world of quantum field theories, two main themes of lowdimensional topology that remained essentially disjoint since their spectacular developments in the early eighties. In particular, the celebrated Kashaev's Volume Conjecture for hyperbolic knots in $S^{3}$ [20] appears as a special instance of the challenging general 
problem of understanding the relationships between the asymptotic behavior when $N \rightarrow \infty$ of QHFT partition functions and fundamental invariants coming from differential geometry, like the Cheeger-Chern-Simons class [3, Section 5; 4, Section 7].

We plan to face the asymptotics of QHFT partition functions in future works. Also, we postpone to a sequel to this paper our study of the relations between the QHFT and Turaev's Homotopic QFT [31], as well as formulas describing the behavior of the QHFT amplitudes under framing changes. Both rely heavily on $R$-matrix computations.

We refer to Benedetti and Bonsante [6] for a discussion of QHG in the framework of gravity in dimension 3 .

Here is the content of the paper. The universal QHG functors are defined in Section 2, where we recall also from [3; 4] and [23; 24] (in Section 2.4 in particular) the notions and results we need.

The QHFT bordism category is described in Section 4, while its objects, the QHFT surfaces, are developed starting with Section 3. The QHFT functors are defined in Section 5. This includes the construction of the distinguished QHG triangulated pseudomanifolds associated to any triple $\left(Y, L_{\mathcal{F}}, \rho\right)$ with marked boundary components, and of the trace tensors computed on them. The conjugacy classes of moderately projective representations of the mapping class groups are treated in Section 5.4.

The partition functions $\mathcal{H}_{N}\left(W, L_{\mathcal{F}}, \rho\right)$ are considered in Section 6. We concentrate on the case when $N>1$, which is technically harder because of the use of global charges in the definition of the trace tensors. However, the results can be repeated almost verbatim to define new Cheeger-Chern-Simons invariants.

We show in Section 6.1 that when $\rho$ is defined on the whole of $W, \mathcal{H}_{N}\left(W, L_{\mathcal{F}}, \rho\right)$ coincides with $H_{N}\left(W, L \cup L^{\prime}, \rho\right)$, where $L^{\prime}$ is a parallel copy of the unframed link $L$ given by the framing $\mathcal{F}$. In Section 6.2 we prove the surgery formula for quantum hyperbolic invariants of cusped manifolds and QHFT partition functions. In fact the QHG pseudomanifolds used to compute the trace tensors carry certain cohomological weights (see Section 2.4) and the partition function values actually depend also on them. These weights play indeed a subtle role in the surgery formulas. This eventually leads to realize $H_{N}(M)$ as the limit of $H_{N}\left(M_{n}, L_{n}, \rho_{n}\right)$, where $M_{n}$ is a sequence of closed hyperbolic manifolds converging geometrically to $M, L_{n}$ is the link of geodesic cores of the hyperbolic Dehn fillings of $M$ that produce $M_{n}$, and $\rho_{n}$ is the hyperbolic holonomy of $M_{n}$. In Section 6.3 we discuss alternative computations of the QHFT partition functions for manifolds that fiber over $S^{1}$. For fibred cusped manifolds, this allows in particular to identify each $H_{N}(M)$ with a special instance of QHFT partition function. We conclude with an example in Section 6.4. 
Acknowledgement The authors thank Charles Frohman for his reading and suggestions for the paper.

\section{Universal QHG}

\subsection{Building blocks}

The building blocks of QHG are flat/charged $\mathcal{I}$-tetrahedra $(\Delta, b, w, f, c)$, and matrix dilogarithms $\mathcal{R}_{N}(\Delta(b, w, f, c)) \in \operatorname{Aut}\left(\mathbb{C}^{N} \otimes \mathbb{C}^{N}\right)$ defined for every odd positive integer $N$, which were introduced in [4].

2.1.1 Flat/charged- $\mathcal{I}$ tetrahedra Consider the half-space model of the oriented hyperbolic space $\mathbb{M}^{3}$, with the group of direct isometries identified with $\operatorname{PSL}(2, \mathbb{C})$

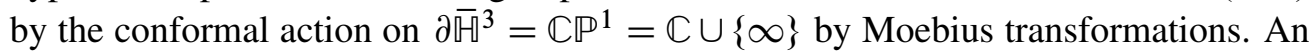
$\mathcal{I}$-tetrahedron is an oriented ideal tetrahedron $\Delta$ in $\partial \overline{\mathbb{T}}^{3}$ with distinct ordered vertices $v_{0}, v_{1}, v_{2}$ and $v_{3}$ on $\partial \overline{\mathbb{T}}^{3}$.

In fact we consider $\Delta$ as an abstract oriented simplex equipped with an additional decoration. The ordering of the vertices is encoded by a branching $b$, that is, edge orientations obtained via the rule: each edge points towards the biggest end-point. We order the 2 -faces $\delta_{0}, \ldots \delta_{3}$ by the opposite vertices, and the edges $e_{0}, e_{1}, e_{2}$ of $\delta_{3}$ by stipulating that for $j=0,1, v_{j}$ is the first end-point of $e_{j}$. Each 2 -face has an induced branching, and a $b$-orientation, which is just compatible with that of two edges on the boundary. For exactly two 2 -faces the $b$-orientation and the boundary orientation are the same, where the boundary orientation is oriented via the convention: last in the ingoing normal. The $b$-orientation of $\Delta$ coincides with the given one if the $b$-orientation of $\delta_{3}$ looks anti-clockwise from $v_{3}$. We give $\Delta$ and each 2-face $\delta$ a $b$-sign $*_{b}$ and $\sigma(\delta)$, which is 1 if the given and the boundary orientations agree, respectively, with the $b$-orientation, and -1 otherwise.

The hyperbolic structure is encoded by the cross-ratio moduli that label the edges of $\Delta$. Recall that opposite edges share the same cross-ratio moduli. We set $w=\left(w_{0}, w_{1}, w_{2}\right)$ with $w_{j}=w\left(e_{j}\right) \in \mathbb{C} \backslash\{0,1\}$. Hence $w_{j+1}=1 /\left(1-w_{j}\right)($ indices $\bmod (\mathbb{Z} / 3 \mathbb{Z}))$, and

$$
w_{0}=\left(v_{2}-v_{1}\right)\left(v_{3}-v_{0}\right) /\left(v_{2}-v_{0}\right)\left(v_{3}-v_{1}\right) .
$$

We say that the $\mathcal{I}$-tetrahedron $(\Delta, b, w)$ is nondegenerate if it is of nonzero volume, that is if the imaginary part of each $w_{i}$ is not zero; then they have the same sign $* w= \pm 1$.

It is very convenient to encode $(\Delta, b, w)$ in dual terms. In Figure 1 we show the 1 -skeleton of the dual cell decomposition of $\operatorname{Int}(\Delta)$ (the indices $i, j, k$ and $l$ are 
considered below). Note that an oriented edge of this 1-skeleton is outgoing exactly when the $b$-sign of the dual 2 -face is 1 , and that the over/under crossing made by these edges also encodes the $b$-sign of the tetrahedron (see [4, Section 2.1.2] for more details).
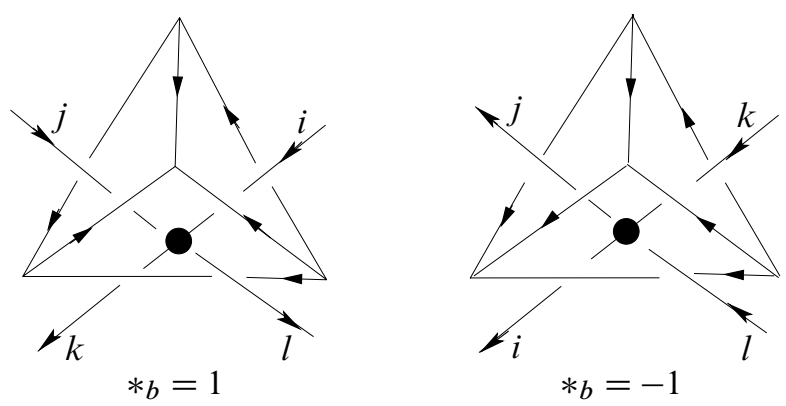

Figure 1: $\mathcal{I}$-tetrahedra and dual encoding

A flat/charged $\mathcal{I}$-tetrahedron is an $\mathcal{I}$-tetrahedron equipped with a flattening $f$ and a charge $c$, two notions first introduced by Neumann [23; 24]. Flattenings and charges are $\mathbb{Z}$-valued functions defined on the edges of $\Delta$ that take the same value on opposite edges and satisfy the following properties, respectively (where log has the imaginary part in $]-\pi, \pi]$ ):

(F) Flattening condition: $1_{0}+1_{1}+1_{2}=0$, where

$$
1_{j}=1_{j}(b, w, f)=\log \left(w_{j}\right)+\sqrt{-1} \pi f_{j},
$$

(C) Charge condition: $c_{0}+c_{1}+c_{2}=1$.

We call $1_{j}$ a (classical) log-branch. For every odd $N>1$ we define the (level $N$ ) quantum log-branch as

$$
1_{j, N}=\log \left(w_{j}\right)+\sqrt{-1} \pi(N+1)\left(f_{j}-*_{b} c_{j}\right) .
$$

The bijective map

$$
\left(1_{0}, 1_{1}\right) \longmapsto\left(w_{0} ; \frac{1_{0}-\log \left(w_{0}\right)}{\sqrt{-1} \pi}, \frac{1_{1}-\log \left(w_{1}\right)}{\sqrt{-1} \pi}\right)
$$

yields an identification of the set of $\log$-branches on $(\Delta, b)$ with the Riemann surface $\widehat{\mathbb{C}}$ of the maps

$$
\varphi_{\varepsilon, \varepsilon^{\prime}}: w_{0} \longmapsto\left(\log \left(w_{0}\right)+\varepsilon \pi \sqrt{-1}, \log \left(\left(1-w_{0}\right)^{-1}\right)+\varepsilon^{\prime} \pi \sqrt{-1}\right)
$$


where $\varepsilon, \varepsilon^{\prime} \in\{0,1\}$ (there are four connected components). The space $\widehat{\mathbb{C}}$ is a maximal abelian covering of $\mathbb{C} \backslash\{0,1\}$, and is explicitly given by

$$
\widehat{\mathbb{C}}=\left(\mathfrak{D} \times \mathbb{Z}^{2}\right) / \sim
$$

where $\mathfrak{D}:=\mathbb{C} \backslash((-\infty ; 0) \cup(1 ;+\infty))$, and

$$
\begin{array}{ll}
(x+i 0 ; p, q) \sim(x-i 0 ; p+2, q) & \text { if } x \in(-\infty ; 0) \\
(x+i 0 ; p, q) \sim(x-i 0 ; p, q+2) & \text { if } x \in(1 ;+\infty) .
\end{array}
$$

Similarly, the set of triples $\left(w_{0}^{\prime}, w_{1}^{\prime}, w_{2}^{\prime}\right)$ with $w_{j}^{\prime}=\exp \left(1_{j, N} / N\right)$ gets identified with the covering $\widehat{\mathbb{C}}_{N}$ of $\mathbb{C} \backslash\{0,1\}$ obtained by taking the quotient of $\widehat{\mathbb{C}}$ with the subgroup $N \mathbb{Z} \times N \mathbb{Z}$ of the deck transformations.

The space $\widehat{\mathbb{C}}$ is isomorphic to the moduli space of similarity classes of triangles in the complex plane, each corner being endowed with a lift to $\mathbb{R}$ of one of the two dihedral angles between the corresponding pair of edges.

The key geometric object on $\widehat{\mathbb{C}}$ is the smooth tangent 2 -vector $\Omega \in \Lambda^{2} T \widehat{\mathbb{C}}$ given by (see Choi [12])

$$
\Omega\left(d \log (z), d \log (1-z)^{-1}\right)=1 .
$$

Here we regard $d \log (z)=z^{-1} d z$ as a covector on $\mathbb{R}^{2}$, and similarly for $d \log (1-z)^{-1}$ and $d \log \left(1-z^{-1}\right)$. These covectors are never zero, and

$$
d \log (z)+d \log (1-z)^{-1}+d \log \left(1-z^{-1}\right)=0
$$

is the only relation that they satisfy over $\mathbb{R}$. Denote by $w$ the dual 2-form:

$$
\begin{aligned}
w & =d \log (z) \wedge d \log (1-z)^{-1} \\
& =2(\operatorname{Re}(1 / z) \operatorname{Im}(1 /(1-z))-\operatorname{Im}(1 / z) \operatorname{Re}(1 /(1-z))) d x \wedge d y,
\end{aligned}
$$

where $z=x+i y$, and consider the complex analytic 1 -form given by

$$
\theta=\frac{1}{2}\left(1_{0} d l_{1}-l_{1} d l_{0}\right)
$$

with $l_{0}$ and $l_{1}$ as in (2-1). Because of (2-6) and the flattening condition, $\theta$ and $w$ are invariant under any orientation preserving change of the branching of $(\Delta, b)$, and they turn to the opposite under a reversing orientation one. The form $w$ is nondegenerate whenever $\operatorname{Im}(z) \neq 0$, and is compatible with the usual complex structure on $\widehat{\mathbb{C}}$. The hermitian metric of the corresponding Kähler structure is $d s^{2}=|z|^{2}|d z|^{2} / \operatorname{Im}(z /(1-$ $z))$.

The form $w$ is the differential version of the extended complex Dehn invariant of [24]. 
2.1.2 Matrix dilogarithms The matrix dilogarithms are tensor-valued maps

$$
\mathcal{R}_{N}:\{\Delta(b, w, f, c)\} \longrightarrow \operatorname{Aut}\left(\mathbb{C}^{N} \otimes \mathbb{C}^{N}\right)
$$

defined on flat/charged $\mathcal{I}$-tetrahedra, which form a family $\left\{\mathcal{R}_{N}\right\}$ indexed by the odd positive integers $N$. In fact $\mathcal{R}_{1}$ is scalar-valued and is obtained from the classical dilogarithm functions; we consider it formally as a 1 by 1 matrix. For each branched tetrahedron $(\Delta, b)$ the tensors $\mathcal{R}_{N}(\Delta(b, w, f, c)), N>1$, define a map on the Riemann surface $\widehat{\mathbb{C}}_{N}$, while $\mathcal{R}_{1}(\Delta, b)$ is defined on the covering $\widehat{\mathbb{C}}$.

In this section we collect formulas for $\left\{\mathcal{R}_{N}\right\}$. We denote by log the natural logarithm, which has the imaginary part in $]-\pi, \pi]$.

The classical matrix dilogarithm $(N=1)$ Following Neumann [24], consider the map $\mathcal{R}: \hat{\mathbb{C}} \rightarrow \mathbb{C} / \pi^{2} \mathbb{Z}$ given by

$$
\begin{aligned}
\mathcal{R}(z ; p, q)=-\frac{1}{2} \int_{0}^{z}\left(\frac{\log (1-t)}{t}+\right. & \left.\frac{\log (t)}{1-t}\right) d t \\
& +\frac{\pi i}{2}(p \log (1-z)+q \log (z))-\frac{\pi^{2}}{6} .
\end{aligned}
$$

By setting $p=q=0$ we recover the classical Rogers dilogarithm function (shifted by $-\pi^{2} / 6$ so that $\left.\mathcal{R}(1)=0\right)$, which is complex analytic on $\mathbb{C} \backslash(-\infty ; 0) \cup(1 ;+\infty)$. We can write

$$
\mathcal{R}(z ; p, q)=-\frac{\pi^{2}}{6}-\int_{0}^{(z ; p, q)} \theta
$$

where the 1 -form $\theta$ is as in (2-7) and the integration is along any path in $\widehat{\mathbb{C}}$ from 0 to $(z ; 0,0)$, say, and then $(z ; 0,0)$ to $(z ; p, q)$. The relation with hyperbolic volume is as follows: for any oriented hyperbolic ideal tetrahedron $\Delta$ with cross-ratio moduli $z$, $1 /(1-z)$ or $1-1 / z$ we have

$\operatorname{Im}(R(z ; p, q))-\operatorname{Vol}(\Delta)=\frac{1}{2}\left(\log |z|\left(\arg (1-z)^{-1}+q \pi\right)+(\arg (z)+p \pi) \log |1-z|\right)$.

The right-hand side is related to the "imaginary part" of the complex Dehn invariant (see Neumann [24] or Dupont and Zickert [13] for details).

The map $\mathcal{R}: \hat{\mathbb{C}} \rightarrow \mathbb{C} / \pi^{2} \mathbb{Z}$ is holomorphic, and lifts to a map with values in $\mathbb{C} / 2 \pi^{2} \mathbb{Z}$ on the component with even-valued flattenings [24, Propositions 2.5-8.1]. We define the classical matrix dilogarithm $\mathcal{R}_{1}$ of flattened $\mathcal{I}$-tetrahedra (hence without integral charge $c$ ) by

$$
\mathcal{R}_{1}(\Delta, b, w, f)=\exp \left(\frac{*_{b}}{\pi \sqrt{-1}} \mathcal{R}\left(w_{0} ; f_{0}, f_{1}\right)\right)
$$


Note that $*_{b} \mathcal{R}\left(w_{0} ; f_{0}, f_{1}\right)$ is invariant under any change of the branching $b$, up to the addition of integer multiples of $\pi^{2} / 6$ [4, Section 4.2].

The quantum matrix dilogarithms $(N>1)$ These depend on a primitive $N$-th root of unity $\zeta$, with $N$ odd, that we fix once and for all as $\zeta=\exp (2 i \pi / N)$ (see Remark 2.1).

Consider the complex-valued function $g$ given by (we put $x^{1 / N}=\exp (\log (x) / N)$ and $0^{1 / N}=0$ )

$$
g(x):=\prod_{j=1}^{N-1}\left(1-x \zeta^{-j}\right)^{j / N}
$$

and set $h(x):=g(x) / g(1)$ (we have $|g(1)|=N^{1 / 2}$ ). It is analytic on the cut complex plane $\mathbb{C} \backslash\left\{r \zeta^{k}, r \geq 1, k=1, \ldots, N-1\right\}$. For any $u^{\prime}, v^{\prime} \in \mathbb{C}$ satisfying $\left(u^{\prime}\right)^{N}+\left(v^{\prime}\right)^{N}=1$ and any $n \in \mathbb{N}$, let

$$
\omega\left(u^{\prime}, v^{\prime} \mid n\right)=\prod_{j=1}^{n} \frac{v^{\prime}}{1-u^{\prime} \zeta^{j}}
$$

with $\omega\left(u^{\prime}, v^{\prime} \mid 0\right)=1$ by convention. The functions $\omega$ are periodic in their integer argument, with period $N$. Write $[x]=N^{-1}\left(1-x^{N}\right) /(1-x)$. Given a flat/charged $\mathcal{I}$-tetrahedron $(\Delta, b, w, f, c)$, set

$$
w_{j}^{\prime}=\exp \left(1_{j, N} / N\right)
$$

with $1_{j, N}$ as in (2-2). Put the standard tensor product basis on $\mathbb{C}^{N} \otimes \mathbb{C}^{N}$. The matrix dilogarithm of level $N>1$ is the tensor-valued function of flat/charged $\mathcal{I}$-tetrahedra defined by

$$
\begin{aligned}
\mathcal{R}_{N}(\Delta, b, w, f, c)=\left(\left(w_{0}^{\prime}\right)^{-c_{1}}\left(w_{1}^{\prime}\right)^{c_{0}}\right)^{\frac{N-1}{2}}\left(\mathcal{L}_{N}\right)^{*_{b}}\left(w_{0}^{\prime},\left(w_{1}^{\prime}\right)^{-1}\right) \\
\\
\in \operatorname{Aut}\left(\mathbb{C}^{N} \otimes \mathbb{C}^{N}\right)
\end{aligned}
$$

where

$$
\begin{aligned}
\mathcal{L}_{N}\left(u^{\prime}, v^{\prime}\right)_{k, l}^{i, j} & =h\left(u^{\prime}\right) \zeta^{k j+(m+1) k^{2}} \omega\left(u^{\prime}, v^{\prime} \mid i-k\right) \delta(i+j-l) \\
\left(\mathcal{L}_{N}\left(u^{\prime}, v^{\prime}\right)^{-1}\right)_{k, l}^{i, j} & =\frac{\left[u^{\prime}\right]}{h\left(u^{\prime}\right)} \zeta^{-i l-(m+1) i^{2}} \frac{\delta(k+l-j)}{\omega\left(u^{\prime} / \zeta, v^{\prime} \mid k-i\right)}
\end{aligned}
$$

Here we put $m=(N-1) / 2$, and $\delta$ is the Kronecker symbol with period $N$, that is $\delta(n)=1$ if $n \equiv 0 \bmod (N)$, and $\delta(n)=0$ otherwise. Note that we use the branching in order to associate an index among $i, j, k$ and $l$ to each 2 -face of $\Delta$. The rule is shown in Figure 1. 
By using properties of the functions $g$ and $\omega$ [4, Lemma 8.2 and Lemma 5.4] we can write the matrix dilogarithm entries in various ways. For instance we have

$$
\begin{aligned}
\left(\mathcal{L}_{N}\right)\left(w_{0}^{\prime},\left(w_{1}^{\prime}\right)^{-1}\right)_{k, l}^{i, j} & =\frac{g\left(w_{0}^{\prime} \zeta^{i-k}\right)}{g(1)} \zeta^{k j+(m+1)\left(k^{2}+(k-i)\left(f_{1}-c_{1}\right)\right)} \delta(i+j-l) \\
& =\frac{g\left(w_{0}^{\prime}\right)}{g(1)}(-q)^{2 k l-i^{2}} \prod_{n=1}^{k-i}\left(\left(w_{2}^{\prime}\right)^{-1}-w_{1}^{\prime} q^{2 n-1}\right) \delta(i+j-l)
\end{aligned}
$$

where $q=-\zeta^{m+1}=\exp (\sqrt{-1} \pi / N)$. Note the main role played by the function $g$ in the first expression, while the second shows a polynomial in $q$.

Because $w_{j}^{\prime}=\exp \left((1 / N)\left(1_{j}-*_{b} \pi i c_{j}\right)\right) \exp \left(\left(f_{j}-*_{b} c_{j}\right) \pi i\right)$ and the parity of $f_{j}$ is unaltered when we move $l_{j}$ continuously, the entries of $\mathcal{R}_{N}(\Delta, b, w, f, c)$ define holomorphic functions on $\widehat{\mathbb{C}}$ (whence on $\widehat{\mathbb{C}}_{N}$ ), up to multiplication by $N$-th roots of unity coming from the discontinuities of $g$ along the cuts $\left\{r \zeta^{k}, r \geq 1, k=1, \ldots, N-1\right\}$.

The map $\mathcal{R}_{N}$ also satisfies some symmetry properties, with a sign ambiguity coming from the factor $\left(\left(w_{0}^{\prime}\right)^{-c_{1}}\left(w_{1}^{\prime}\right)^{c_{0}}\right)^{(N-1) / 2}$ and the relation $w_{0}^{\prime} w_{1}^{\prime} w_{2}^{\prime}=-\zeta^{-*_{b}(m+1)}$, and an $N$ root ambiguity due to the branch cuts of the function $g$ in (2-10); see [4, Proposition 5.3 and Corollary 5.6].

Remark 2.1 The choice $\zeta=\exp (2 i \pi / N)$ is the one used in our previous papers $[3 ; 4]$. However, the matrix dilogarithms satisfy the very same fundamental properties (stated in Sections 5 and 8 of [4]) by replacing $\zeta$ with any primitive $N$-th root of unity $\zeta_{d}=\exp (2 d i \pi / N)$ if we simultaneously put $w_{j}^{\prime}=\exp \left(d 1_{j, N} / N\right)$ in (2-11). In fact there is no other possibility for $w_{j}^{\prime}$ : the inversion formula of $\mathcal{R}_{N}$ takes the same form at the root $\zeta_{d}$ [4, Proposition 8.6], so that the symmetry relations of $\mathcal{R}_{N}$ are satisfied only if $w_{0}^{\prime} w_{1}^{\prime} w_{2}^{\prime}=-\zeta_{d}^{-*_{b}(m+1)}$ (see the computation at the top of $\mathrm{p} 528$ of [4]). This, in turn, implies $w_{j}^{\prime}=\exp \left(d 1_{j, N} / N\right)$. (Another instance of this argument is detailed at the bottom of p 532 of [4]).

Matrix dilogarithms via geometric quantization The quantum matrix dilogarithms were derived in the appendices of [3; 4] from the Kashaev's 6j-symbols of the cyclic representation theory of a Borel quantum subalgebra $\mathcal{B}_{\zeta}$ of $U_{\zeta}(s l(2, \mathbb{C}))$, where $\zeta=$ $\exp (2 i \pi / N)$. Let us outline here very briefly an alternative construction based on the geometric quantization of the classical dilogarithm $\mathcal{R}_{1}$, that may shed some light on their geometric nature and the results of Section 3.3 and Section 5.4 (for details see Baseilhac and Benedetti [5]).

Let us regard $\mathbb{C}$ as the Riemann surface of log, ie

$$
\mathbb{C}=\left\{(z ; p) \in \mathbb{C}^{*} \times 2 \mathbb{Z}\right\} /((z+i 0 ; p) \sim(z-i 0 ; p+2), \text { for all } z \in(-\infty ; 0)) .
$$


Similarly as in $(2-1)$, set $1(z ; p)=\log (z)+\sqrt{-1} \pi p$. Consider the space

$$
X=\left\{(u, v, w) \in\left(\mathbb{C}^{*}\right)^{3} \mid u v w=-1\right\}
$$

and put

$$
\widehat{X}=\left\{((u ; p),(v ; q),(w ; r)) \in \mathbb{C}^{3} \mid 1(u ; p)+1(v ; q)+1(w ; r)=0\right\} .
$$

We can identify $X$ with $\mathbb{C}^{*} \times \mathbb{C}^{*}$ and $\hat{X}$ with $\mathbb{C}^{2}$ via the first two coordinates. Define

$$
\begin{array}{ll}
b_{X}=d \log u \wedge d \log v & \in \Lambda^{2,0} T^{*} X \\
\theta_{X}=\frac{1}{2}((1(u ; p) d \log v-1(v ; q) d \log u) & \in \Lambda^{1,0} T^{*} \widehat{X} .
\end{array}
$$

Note that the one-form $\theta_{X}$ is holomorphic, and is a primitive of the lift of $b_{X}$ to $\hat{X}$. Consider the connected component $\widehat{\mathbb{C}}_{0,0}$ of $\widehat{\mathbb{C}}$ with even-valued flattenings, ie the Riemann surface of the map $\varphi_{0,0}$ in (2-4). We have an embedding

$$
\begin{aligned}
\widehat{\mathbb{C}}_{0,0} & \longrightarrow \hat{X} \\
(u ; p, q) & \longmapsto\left(1(u ; p), 1\left((1-u)^{-1} ; q\right)\right)
\end{aligned}
$$

mapping $\widehat{\mathbb{C}}_{0,0}$ onto a submanifold of $\left(\hat{X}, b_{X}\right)$ which is Lagrangian. In fact the restriction of $b_{X}$ to this submanifold gets identified with the linear extension to the complexified tangent bundle $T\left(\widehat{\mathbb{C}}_{0,0}\right)_{\mathbb{C}}$ of the symplectic form $w$ on $\widehat{\mathbb{C}}_{0,0}$. Geometrically, we can view $\hat{X}$ as the tangent space at the identity of the group of complex plane similarities, the projective Borel group $P B(2, \mathbb{C})$.

Consider an abstract oriented quadrilateral $Q$, triangulated by two triangles. Order the triangles of $Q$, and associate to each a copy $X_{i}, i=1,2$, of the space $X$ in (2-13), where $u, v$ and $w$ correspond to the triangle corners, ordered cyclically by using the orientation. Consider the quadrilateral $Q^{\prime}$ obtained from $Q$ by exchanging the diagonal, and fix any ordering of its triangles. Denote by $S_{Q}^{2}$ the 2 -sphere obtained by gluing $Q$ and $Q^{\prime}$ along the boundary in the natural way. Any triangle of $S_{Q}^{2}$ is uniquely determined by its vertex set, so the permutation group of the vertices of $S_{Q}^{2}$ acts naturally by permutation on the spaces $X_{i}$ attached to the triangles of $S_{Q}^{2}$, $i=1, \ldots, 4$. Moreover, on each triangle it permutes the coordinates.

Define $b_{Q}=b_{X_{1}}+b_{X_{2}}$ and $\theta_{Q}=\theta_{X_{1}}+\theta_{X_{2}}$, where $b_{X_{i}}$ and $\theta_{X_{i}}$ are as in (2-14) and correspond to the space $X_{i}$, and similarly for $b_{Q^{\prime}}$ and $\theta_{Q^{\prime}}$. Note that they are invariant under any even permutation of the vertices of $Q$, and turn to the opposite under odd ones. We regard $\left(\hat{X}_{1} \times \hat{X}_{2}, b_{Q}\right)$ and $\left(\hat{X}_{3} \times \widehat{X}_{4}, b_{Q^{\prime}}\right)$ as complex symplectic spaces attached to $Q$ and $Q^{\prime}$. Consider the family $\left\{\tilde{\phi}_{Q}^{n}\right\}_{n \in \mathbb{Z}}$ of maps

$$
\widetilde{\phi}_{Q}^{n}:\left(\hat{X}_{1} \times \hat{X}_{2}, b_{Q}\right) \longrightarrow\left(\hat{X}_{3} \times \widehat{X}_{4}, b_{Q^{\prime}}\right)
$$


given by $\tilde{\phi}_{Q}^{n}\left(\left(1\left(u_{1} ; p_{1}\right), 1\left(v_{1} ; q_{1}\right)\right),\left(1\left(u_{2} ; p_{2}\right), 1\left(v_{2} ; q_{2}\right)\right)\right)$

$$
=\left(\left(1\left(u_{1}^{\prime} ; p_{1}^{\prime}\right), 1\left(v_{1}^{\prime} ; q_{1}^{\prime}\right)\right),\left(1\left(u_{2}^{\prime} ; q_{2}^{\prime}\right), 1\left(v_{2}^{\prime} ; q_{2}^{\prime}\right)\right)\right),
$$

where (see Figure 2)

$$
\left\{\begin{array}{l}
1\left(u_{1}^{\prime} ; p_{1}^{\prime}\right)=1\left(u_{1} ; p_{1}\right)-1\left(u_{2}^{\prime} ; p_{2}^{\prime}\right) \\
1\left(v_{1}^{\prime} ; q_{1}^{\prime}\right)=1\left(v_{1} ; q_{1}\right)+1\left(u_{2} ; p_{2}\right)
\end{array}\right.
$$

$$
\left\{\begin{array}{l}
1\left(u_{2}^{\prime} ; p_{2}^{\prime}\right)=1\left(u_{2} ; p_{2}\right)-1\left(\left(1-u_{1} v_{2}\right)^{-1} ; n\right) \\
1\left(v_{2}^{\prime} ; q_{2}^{\prime}\right)=1\left(v_{2} ; q_{2}\right)+1\left(u_{1}^{\prime} ; p_{1}^{\prime}\right)+1\left(v_{1}^{\prime} ; q_{1}^{\prime}\right)
\end{array}\right.
$$

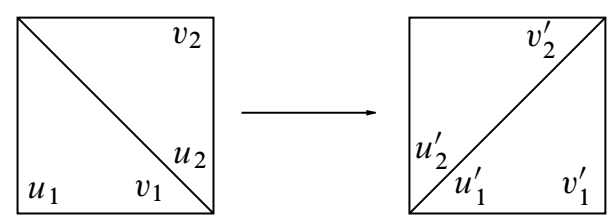

Figure 2: A diagonal exchange

Proposition 2.2 The maps $\widetilde{\phi}_{Q}^{n}$ are holomorphic, equivariant under the group of permutations of the vertices of the sphere $S_{Q}^{2}$, and satisfy $b_{Q}=\left(\tilde{\phi}_{Q}^{n}\right)^{*} b_{Q^{\prime}}$. Moreover, we have the pentagon relation

$$
\tilde{\phi}_{Q_{5}}^{n_{5}} \circ \tilde{\phi}_{Q_{4}}^{n_{4}} \circ \widetilde{\phi}_{Q_{3}}^{n_{3}} \circ \widetilde{\phi}_{Q_{2}}^{n_{2}} \circ \tilde{\phi}_{Q_{1}}^{n_{1}}=\operatorname{Id}_{\hat{X}^{3}}
$$

for any $n_{i} \in \mathbb{Z}$, where $Q_{i}$ has diagonal the $i$-th edge exchanged in Figure 3, for the positive cyclic ordering of the pentagons starting from the top left one.

The maps $\widetilde{\phi}_{Q}^{n}$ can be characterized as the unique (up to scalar factors) equivariant holomorphic maps which preserve the sums of coordinates at the quadrilateral corners. Geometrically, if $\Sigma$ is a pleated hyperbolic punctured surface with pleating locus an ideal triangulation $\lambda$, and $Q$ is realized as the abstract star of an edge of $\lambda$, the maps $\tilde{\phi}_{Q}^{n}$ encode the change of tangent vectors at the exponential shear-bend coordinates of the boundary edges of $Q$ when we change the diagonal to get $Q^{\prime}$, that is, when we glue a flattened hyperbolic ideal tetrahedron to $\Sigma$. We can see this (for instance in the third identity in (2-16)) by noting that the diagonal of $Q$ is associated a copy of the space $\widehat{\mathbb{C}}$, namely

$$
\widehat{\mathbb{C}}\left(u_{1} v_{2} ; m, n\right)=\left\{\left(1\left(u_{1} ; p_{1}\right)+1\left(v_{2} ; q_{2}\right), 1\left(\left(1-u_{1} v_{2}\right)^{-1} ; n\right)\right)\right\},
$$




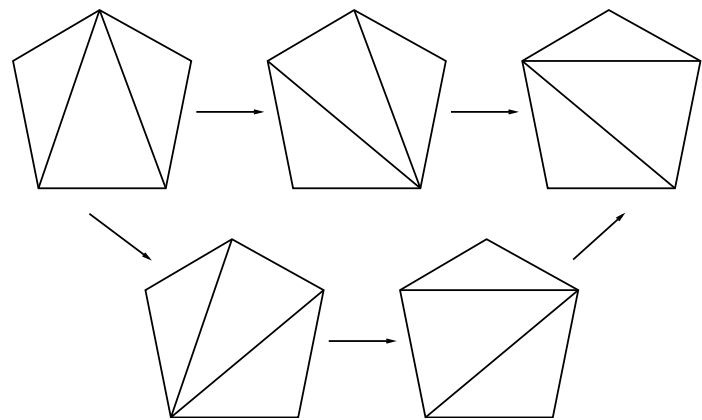

Figure 3: The pentagon relation

where $n$ is specified by $\widetilde{\phi}_{Q}^{n}$ and $m=\left(\log \left(u_{1} v_{2}\right)-1\left(u_{1} ; p_{1}\right)-1\left(v_{2} ; q_{2}\right)\right) / \sqrt{-1} \pi$. In particular, the pentagon relation (2-17) corresponds to a $2 \leftrightarrow 3$ QHG transit. The choice of coordinates of the various spaces $X_{i}$ determines the branching, and the integers $n_{i}$ in the $\widetilde{\phi}_{Q_{i}}^{n_{i}}$ fix the transit of flattenings. In this setting no charge is needed.

Since $b_{Q}-b_{Q}^{\prime}$ vanishes identically on the graph of $\widetilde{\phi}_{Q}^{n}$, the 1 -form $\theta_{Q}-\left(\tilde{\phi}_{Q}^{n}\right)^{*} \theta_{Q^{\prime}}$ should be locally the differential of some holomorphic function on $\hat{X}_{1} \times \hat{X}_{2}$. In fact we easily check the following:

Lemma 2.3 We have $\theta_{Q}-\left(\tilde{\phi}_{Q}^{n}\right)^{*} \theta_{Q^{\prime}}=-d \mathcal{R}$, where $\mathcal{R}: \hat{\mathbb{C}}\left(u_{1} v_{2} ; m, n\right) \rightarrow \mathbb{C} / 2 \pi^{2} \mathbb{Z}$ is the extended dilogarithm defined in (2-9).

The embedding (2-15) and the form $b_{X}$ can be used to derive the combinatorial formulas obtained in Papadapoulos and Penner [25] (see also Bonahon [8] and Bonahon and Sözen [29]) for the Thurston's intersection form, which is a complex antisymmetric bilinear form on the shear-bend parameter spaces of pleated hyperbolic punctured surfaces. In this setting $\theta_{X}$ encodes the complex length differential form of [5, Section 11]. By Lemma 2.3, $\mathcal{R}$ encodes the variation of the latter under shear-bend coordinate changes induced by changes of the triangulation.

Consider the four copies of (2-15) corresponding to the $X_{i}$, where we replace $\widehat{\mathbb{C}}_{0,0}$ with its quotient $\left(\widehat{\mathbb{C}}_{0,0}\right)_{N}$, defined as at the end of Section 2.1.1, endowed with the form $N w$ and its primitive $N \theta$. We can apply the technics of geometric quantization to produce an $N$-dimensional vector space $\Gamma^{N}$ of holomorphic sections of a line bundle over $\left(\widehat{\mathbb{C}}_{0,0}\right)_{N}$, the coherent states. This construction behaves well with respect to branchings if we use half forms, which are encoded by charges. Then, the maps $\widetilde{\Phi}_{Q}^{n}: \Gamma_{1}^{N} \otimes \Gamma_{2}^{N} \rightarrow \Gamma_{3}^{N} \otimes \Gamma_{4}^{N}$ induced by pull back via $\left\{\widetilde{\phi}_{Q}^{n}\right\}$, that is such that $\widetilde{\Phi}_{Q}^{n}(s)=$ 
$s \circ \widetilde{\phi}_{Q}^{n}$ for any $s \in \Gamma_{1}^{N} \otimes \Gamma_{2}^{N}$, coincide with the matrix dilogarithms $\mathcal{R}_{N}$ for some suitable basis of the $\Gamma_{i}^{N}$.

Hence the matrix dilogarithms relate the sections of some line bundles over spaces of cross-ratios. In this setting the flattenings have to do with the coordinates on the base space, while the charges have to do with the sections.

\subsection{QHG triangulated pseudomanifolds}

We restrict the discussion to pseudomanifolds for simplicity, but all what follows makes sense for arbitrary singular 3-cycles whose nonmanifold locus is of codimension $\geq 2$. By a pseudomanifold $Z$, possibly with nonempty boundary $\partial Z$, we mean a compact oriented polyhedron with at most a finite set of nonmanifold points. The boundary is a pseudo-surface.

A $Q H G$ triangulated pseudomanifold $(Z, \mathcal{T})$ is a pseudomanifold $Z$ obtained as the quotient of a finite family $\mathcal{Z}=\left\{\left(\Delta^{i}, b^{i}, w^{i}, f^{i}, c^{i}\right)\right\}$ of flat/charged $\mathcal{I}$-tetrahedra, via a system of orientation reversing simplicial identifications of pairs of 2-faces such that the branchings match. The resulting triangulation $T$ of $Z$ is endowed with a global branching $b$, and is possibly singular (multiple adjacent as well as self adjacent tetrahedra are allowed). The set of nonmanifold points of $Z$ is contained in the set of vertices of $T$. We do not impose for the moment any global constraint on the moduli, flattening and charges. Hence $Z$ is equipped with a rough flat/charged $\mathcal{I}$-triangulation $\mathcal{T}=(T, b, w, f, c)$, where $w=\left\{w^{i}\right\}$ and so on.

Next we define the QHG triangulated pseudomanifold isomorphisms. Fix a QHG triangulated pseudomanifold $(Z, \mathcal{T})$, and let $\epsilon_{T}: E(\mathcal{Z}) \rightarrow E(T)$ be the identification map of edges. We define the total modulus, log-branch and charge of an edge $e$, respectively, by:

$$
\begin{aligned}
& W_{\mathcal{T}}(e)=\prod_{h \in \epsilon_{T}^{-1}(e)} w(h)^{*_{b}} \\
& L_{\mathcal{T}}(e)=\sum_{h \in \epsilon_{T}^{-1}(e)} *_{b} 1(h) \\
& C_{\mathcal{T}}(e)=\sum_{h \in \epsilon_{T}^{-1}(e)} c(h)
\end{aligned}
$$

where $*_{b}= \pm 1$ according to the $b$-orientation of the tetrahedron in $Z$ that contains $h, w(h)$ is the cross-ratio modulus at $h, l(h)$ the log-branch at $h$, and $c(h)$ the charge at $h$. 
Remark 2.4 It is easily seen that $W_{\mathcal{T}}(e)$ is a cross ratio for the four "extremal" points on $\partial \mathbb{\boxplus}^{3}$ determined by gluing oriented hyperbolic ideal tetrahedra with moduli $w(h)^{*_{b}}$ along a common edge (by continuity $W_{\mathcal{T}}(e)=1$ for a degenerate quadrilateral with three distinct vertices).

It is known that any two arbitrary naked triangulations $T, T^{\prime}$ of $Z$ with the same boundary triangulation can be connected (keeping the boundary triangulation fixed) by a finite sequence of the local moves shown in Figure 4, the $2 \leftrightarrow 3$ move (top) and the bubble move (bottom) (see Turaev and Viro [33, Theorem 3.2.A and Appendix 2]). The bubble move consists in replacing a $2-$ simplex by the cone on a $2-$ sphere triangulated by two 2 -simplices.

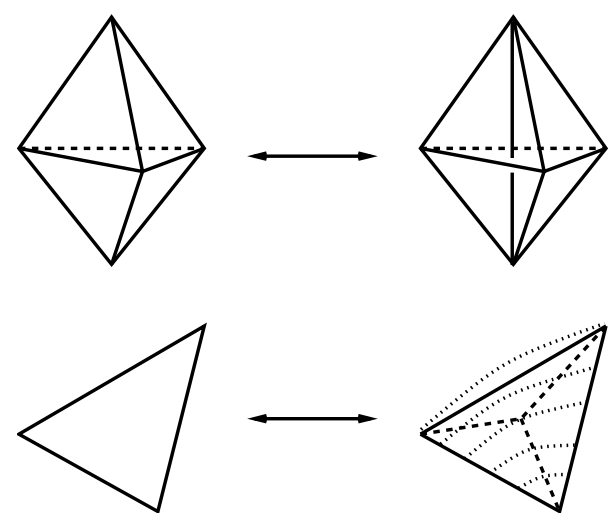

Figure 4: The moves on naked singular triangulations

For any such a local move $T \leftrightarrow T^{\prime}$ we have two triangulations of a same portion of a polyhedron $Q$. Assume that both $T$ and $T^{\prime}$ extend to portions $(Q, \mathcal{T})$ and $\left(Q, \mathcal{T}^{\prime}\right)$ of QHG triangulated pseudomanifolds. We have to specify the admissible $Q H G$ transits $\mathcal{T} \leftrightarrow \mathcal{T}^{\prime}$. In any case we require that they are local (that is, the portions complements remain unchanged), and that the branchings coincide at every common edge of $T$ and $T^{\prime}$.

For the $2 \leftrightarrow 3$ move we also require that at every common edge $e$ as above the total modulus, log-branch and charge coincide:

$$
W_{\mathcal{T}}(e)=W_{\mathcal{T}^{\prime}}(e), \quad L_{\mathcal{T}}(e)=L_{\mathcal{T}^{\prime}}(e), \quad C_{\mathcal{T}}(e)=C_{\mathcal{T}^{\prime}}(e) .
$$

It is easily checked that there is one degree of freedom for choosing flattenings or charges after a $2 \rightarrow 3$ transit. 
The same rule restricted to the total modulus and total log-branch holds also for the bubble move; however, the total charge behaves in a different way: any bubble transit $\mathcal{T} \leftrightarrow \mathcal{T}^{\prime}$ includes a marked edge $e$ common to $T$ and $T^{\prime}$. Referring to the bottom of Figure 4 we require that $C_{\mathcal{T}}(e)=C_{\mathcal{T}^{\prime}}(e)-2$, while for the other two common edges the total charges are unchanged.

Remark 2.5 For every QHG transit supported by a $2 \leftrightarrow 3$ move (top of Figure 4), if $E_{0}$ denotes the new edge in $T^{\prime}$ then

$$
W_{\mathcal{T}^{\prime}}\left(E_{0}\right)=1, L_{\mathcal{T}^{\prime}}\left(E_{0}\right)=0, C_{\mathcal{T}^{\prime}}\left(E_{0}\right)=2 .
$$

For every QHG bubble transit, let $f^{\prime}$ be the unique new 2-simplex of $T^{\prime}$ that contains the marked edge $e$. Denote by $E_{1}$ and $E_{2}$ the other edges of $f^{\prime}$, and by $E_{3}$ the further new edge of $T^{\prime}$. Then we have

$$
\begin{aligned}
& W_{\mathcal{T}^{\prime}}\left(E_{j}\right)=1, \quad L_{\mathcal{T}^{\prime}}\left(E_{j}\right)=0, \quad j=1,2,3 \\
& C_{\mathcal{T}^{\prime}}\left(E_{1}\right)=C_{\mathcal{T}^{\prime}}\left(E_{2}\right)=0, \quad C_{\mathcal{T}^{\prime}}\left(E_{3}\right)=2 .
\end{aligned}
$$

Definition 2.6 A $Q H G$ isomorphism between QHG triangulated pseudomanifolds is any finite composition of QHG transit and oriented simplicial homeomorphisms that preserve the whole decoration.

\subsection{QHG universal functor}

For every odd $N \geq 1$, we associate to every QHG triangulated pseudomanifold $(Z, \mathcal{T})$ a trace tensor $\mathcal{H}_{N}(\mathcal{T})$, as follows. Define an $N$-state of $T$ as a function that gives every 2 -simplex an index, with values in $\{0, \ldots, N-1\}$. Every $N$-state determines an entry for each matrix dilogarithm $\mathcal{R}_{N}(\Delta, b, w, f, c)$. As two tetrahedra induce opposite orientations on a common 2-face, an index is down for the $\mathcal{R}_{N}$ of one tetrahedron while it is up for the other (see Figure 1). By summing over repeated indices we get the total contraction of the tensors $\left\{\mathcal{R}_{N}(\Delta, b, w, f, c)\right\}$, that we denote

$$
\mathbb{} r_{\Delta \subset T} \mathcal{R}_{N}(\Delta, b, w, f, c)=\sum_{s} \prod_{\Delta \subset T} \mathcal{R}_{N}(\Delta, b, w, f, c)_{s}
$$

where the sum is over all $N$-states of $T$ and $\mathcal{R}_{N}(\Delta, b, w, f, c)_{s}$ stands for the matrix dilogarithm entry determined by $s$. Let $v_{I}$ and $v_{\delta}$ be the number of vertices of $T \backslash \partial T$ and $\partial T$, respectively, that correspond to manifold points. We set

$$
\mathcal{H}_{N}(\mathcal{T})=N^{-\left(v_{\delta} / 2+v_{I}\right)} \mathbb{\pi} r_{\Delta \subset T} \mathcal{R}_{N}(\Delta, b, w, f, c)
$$

The type of a trace tensor $\mathcal{H}_{N}(\mathcal{T})$ depends on the $b$-signs of the boundary triangles of $(T, b)$. The matrix dilogarithms themselves are special instances of trace tensors. 
When $N=1$, denoting by $\left(\Delta^{i}, b^{i}, w^{i}, f^{i}, c^{i}\right)$ the flat/charged $\mathcal{I}$-tetrahedra of $\mathcal{T}$, we can write (2-22) as

$$
\begin{aligned}
\mathcal{H}_{N}(\mathcal{T})=\prod_{\Delta^{i} \subset T} \mathcal{R}_{1}\left(\Delta^{i}, b^{i}, w^{i}, f^{i}, c^{i}\right) & \\
& =\exp \left(\frac{1}{\pi \sqrt{-1}} \sum_{\Delta^{i} \subset T} * b^{i} \mathcal{R}\left(w_{0}^{i} ; f_{0}^{i}, f_{1}^{i}\right)\right) .
\end{aligned}
$$

We have the following:

Proposition 2.7 The trace tensors $\mathcal{H}_{N}(\mathcal{T})$ and $\mathcal{H}_{N}\left(\mathcal{T}^{\prime}\right)$ of QHG isomorphic triangulated pseudomanifolds $(Z, \mathcal{T})$ and $\left(Z, \mathcal{T}^{\prime}\right)$ coincide up to sign and multiplication by $N-$ th roots of unity.

This result is a restatement of Theorem 2.1 (2) and Lemma 6.7 of [4], and summarizes the fundamental functional relations satisfied by the matrix dilogarithms. In particular those corresponding to $2 \leftrightarrow 3 \mathrm{QHG}$ transits are usually called five terms identities. In Figure 5 we show one instance in dual terms. The normalization factor $N^{-v_{I}}$ in (2-22) is due to the bubble move, that changes by 1 the number of internal vertices.

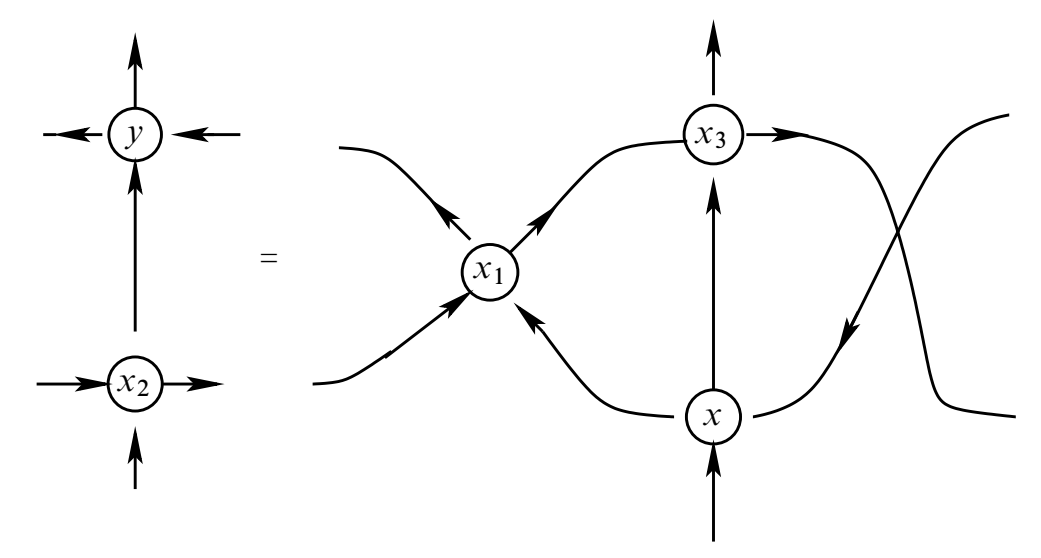

Figure 5: A $2 \leftrightarrow 3$ QHG move $\left(x_{1}=y / x, x_{2}=y(1-x) / x(1-y)\right.$, and $\left.x_{3}=(1-x) /(1-y)\right)$

Remark 2.8 It is shown in [4, Section 5.2] that the quantum matrix dilogarithms satisfy "nice" symmetry relations only when $w_{0}^{\prime} w_{1}^{\prime} w_{2}^{\prime}=-\zeta^{-*_{b}(m+1)}$. This, in turn, forces the use of flattenings and charges for defining the $N$-th root moduli in (2-11). On another hand, the symmetry relations are needed for the whole set of five term 
identities to be true [4, Section 5.3]. A more intrinsic but equivalent way to see the role of charges should be via half-form geometric quantization, as mentioned at the end of Section 2.1.1.

Remark 2.9 The ambiguities in Proposition 2.7 are consequences of the symmetry relations satisfied by the matrix dilogarithms (see before Remark 2.1). We will show in Proposition 2.16 how the sign ambiguity can be gauged out. It could be that the $N$ root ambiguity can be removed by working on the Riemann surface of the function $g$ in (2-10) and using Maslov indices, but the authors have not tried this yet.

The notions of "boundary" (also allowing only portions of the standard boundary see eg Turaev [32]), bordism, and bordism gluing are well defined for the category of QHG triangulated pseudomanifolds considered up to QHG isomorphisms. Hence the association of the trace tensor to each bordism defines a functor. The normalization factor $N^{-v_{\delta} / 2}$ in (2-22) compensates the change in the number of internal vertices when gluing along complete connected components of the (usual) boundary (this is easily adapted to more general gluings).

Any bordism is the gluing of flat/charged $\mathcal{I}$-tetrahedra, considered as elementary bordisms between the couples of 2 -faces having $b$-sign equal to -1 or +1 . The matrix dilogarithms can be interpreted as the amplitudes of a diagonal exchange that relates two quadrilateral triangulations (recall the above discussion about the geometric quantization derivation of matrix dilogarithms.)

\subsection{Towards geometric specializations}

The category of QHG triangulated pseudomanifolds is built ad hoc on the functional properties of the matrix dilogarithms. In order to eventually get specializations with a geometric content, such as QHFT, it is necessary to refine more and more our rough flat/charged $\mathcal{I}$-triangulations. In fact we have to impose some global constraints that would be preserved by (possibly refined) QHG isomorphisms. It is useful to recall at once some of these refinements, and related matter. We adopt the notation introduced above.

Definition 2.10 A triple $\mathcal{T}=(T, b, w)$ is an $\mathcal{I}$-triangulation if at each edge $e$ not contained in $\partial T$ we have the edge compatibility relation $W_{\mathcal{T}}(e)=1$. We say that $\mathcal{T}=(T, b, w, f)$ is a flattened $\mathcal{I}$-triangulation (and $f$ a global flattening) if moreover $L_{\mathcal{T}}(e)=0$. 
It is easily seen that the collection of cross-ratio moduli of an $\mathcal{I}$-triangulation $\mathcal{T}$ of a pseudomanifold $Z$ defines a PL pseudo developing map $d: \widetilde{Z} \rightarrow \overline{\mathbb{H}}^{3}$, unique up to post composition with the action of $\operatorname{PSL}(2, \mathbb{C})$, and a holonomy representation $h: \pi_{1}(Z) \rightarrow P S L(2, \mathbb{C})$ such that $d(\gamma(y))=h(\gamma)(d(y))$ for every $\gamma \in \pi_{1}(Z), y \in \tilde{Z}$.

Our main tool for producing $\mathcal{I}$-triangulations is the following idealization procedure:

Definition 2.11 Let $\mathcal{T}=(T, b, z)$ be a branched triangulation of a pseudomanifold $Z$ equipped with a $\operatorname{PSL}(2, \mathbb{C})$-valued 1 -cocycle $z$, where the cocycle relation is $z\left(e_{0}\right) z\left(e_{1}\right) z\left(e_{2}\right)^{-1}=1$ on a branched triangle with edges $e_{0}, e_{1}$ and $e_{2}$. A 3-simplex $\Delta$ of $(T, b, z)$ with vertices $x_{0}, x_{1}, x_{2}, x_{3}$ is idealizable if

$$
u_{0}=0, u_{1}=z\left(x_{0} x_{1}\right)(0), u_{2}=z\left(x_{0} x_{2}\right)(0), u_{3}=z\left(x_{0} x_{3}\right)(0)
$$

are four distinct points in $\mathbb{C} \subset \mathbb{C P} \mathbb{P}^{1}=\partial \overline{\mathbb{T}}^{3}$. The convex hull is a hyperbolic ideal tetrahedron with ordered vertices, denoted $\left(u_{0}, u_{1}, u_{2}, u_{3}\right)$. Let us give each edge $e$ of $\Delta$ the cross-ratio modulus $w(e) \in \mathbb{C} \backslash\{0,1\}$ of the corresponding edge in $\left(u_{0}, u_{1}, u_{2}, u_{3}\right)$, and put $w=\left(w_{0}, w_{1}, w_{2}\right) \in(\mathbb{C} \backslash\{0,1\})^{3}$. We call $(\Delta, b, w)$ the idealization of $(\Delta, b, z)$. If all 3 -simplices of $(T, b, z)$ are idealizable we say that $\mathcal{T}_{\mathcal{I}}=(T, b, w)$ is the idealization of $\mathcal{T}$.

Note that the idealization includes the choice of a base point (ie 0 in Definition 2.11; we make this choice once for ever). The idealization of each single 3-simplex strongly depends on this choice, but taking a different base point is equivalent to conjugating the cocycle $z$. Not all branched triangulations support idealizable cocycles (for example an ideal triangulation of a knot in $S^{3}$ only supports the trivial constant cocycle). However, when it exists we have:

Lemma 2.12 [3, Section 2.4] The idealization $\mathcal{T}_{\mathcal{I}}$ is an $\mathcal{I}$-triangulation of $Z$.

The following fact will be important below (a version of it was implicitly used in [3]; see (3), Lemma 2.12 and Section 3.2 in that paper):

Lemma 2.13 The idealization $\mathcal{T}_{\mathcal{I}}$ is canonically flattened by taking for each 3-simplex the log-branches (with the notation of Definition 2.11):

$$
\begin{aligned}
& 1_{0}:=\log \left(u_{2}-u_{1}\right)+\log \left(u_{3}\right)-\log \left(u_{2}\right)-\log \left(u_{3}-u_{1}\right) \\
& 1_{1}:=\log \left(u_{2}\right)+\log \left(u_{3}-u_{1}\right)-\log \left(u_{1}\right)-\log \left(u_{3}-u_{2}\right) \\
& 1_{2}:=\log \left(u_{3}-u_{2}\right)+\log \left(u_{1}\right)-\log \left(u_{3}\right)-\log \left(u_{2}-u_{1}\right) .
\end{aligned}
$$

Moreover, canonical flattenings are related by QHG transits, and replacing at each edge of $\mathcal{T}_{\mathcal{I}}$ the standard $\log$ with any other $\log$ determination still makes it flattened. 
Proof These expressions are just signed sums of the standard logs of the edge vectors in the cross-ratio moduli $w_{0}=\left(u_{2}-u_{1}\right) u_{3} / u_{2}\left(u_{3}-u_{1}\right), w_{1}=u_{2}\left(u_{3}-u_{1}\right) / u_{1}\left(u_{3}-u_{2}\right)$ and $w_{2}=-\left(u_{3}-u_{2}\right) u_{1} /\left(u_{2}-u_{1}\right) u_{3}$. They clearly define triples of log-branches. The idealizations for two distinct branchings with the same $b$-orientation are related by an element in $\operatorname{PSL}(2, \mathbb{C})$, that is, a conformal transformation of $\mathbb{C} P^{1}$. We deduce that triples $\left(1_{0}, 1_{1}, l_{2}\right)$ are invariant under a change of branching, because the angle formed by any pair of vectors is preserved (for instance $u_{2}-u_{1}$ and $u_{2}$, or $u_{3}$ and $u_{3}-u_{1}$ ). Then, the edge compatibility relations $L_{\mathcal{T}}(e)=0$ follow easily by developing the tetrahedra around $e$ with branchings such that $e=e_{0}$, similarly as for Lemma 2.12.The last claim is a straightforward checking: in particular note that any log correction appears for each 3 -simplex in two distinct $1_{j}$, with opposite signs.

Remark 2.14 The canonical flattening is even-valued, in the sense that it takes values in $2 \mathbb{Z}$ on the edges $e_{0}$ and $e_{1}$ of each 3-simplex (compare with (2-3)). Note that a general QHG transit need not preserve even-valued flattenings, though a subfamily does (see the comment after (2-19)). This subfamily generates the five term relations defining the "more" extended pre-Bloch group of [24, Section 8] (see also [13]). In fact, by the discussion after Theorem 2.20 below, general flattenings on a given $\mathcal{I}$-triangulation form affine spaces over an explicitly known integral lattice, while even-valued flattenings form affine spaces over a sublattice of finite index $2^{n_{1}}$, where $n_{1}$ is the number of interior edges.

Remark 2.15 The authors see no way to define a notion of canonical charge. In fact such a notion would contradict the need of half forms to construct the quantum matrix dilogarithms via geometric quantization.

Proposition 2.16 The statement of Proposition 2.7 holds true with no sign ambiguity if the flattenings of both $\mathcal{T}$ and $\mathcal{T}^{\prime}$ are even-valued as in Remark 2.14.

As noted in [4, p 532], there is no sign ambiguity for any flattening whenever $N \equiv 1$ $\bmod (4)$ and $N>1$.

Proof By Lemma 6.7 in [4], the result for QHG bubble transits is a consequence of that for $2 \leftrightarrow 3$ QHG transits. There is a preferred "basic" $2 \leftrightarrow 3$ QHG transit for which evenvalued flattenings give no sign ambiguity in Proposition 2.7 (see the proof of Theorem 5.7 in [4] for $N>1$, and Proposition 2.5 and the Appendix in [13] for $N=1$ ). The corresponding branching configuration is defined by ordering the vertices as 1 and 3 on the bottom and top of the central edge, and 0,2, 4 counterclockwise as viewed from the 3 rd vertex. Since all the possible branching configurations of $2 \leftrightarrow 3$ QHG transits 
are obtained, up to obvious symmetries, from the basic one by applying any of the transpositions (01), (12), (23) or (34) of the vertices, we have to check how any such a transposition changes the matrix dilogarithm of each tetrahedron. By Corollary 5.6 of [4], when $N>1$ this action is by matrix conjugation and multiplication by a determined scalar factor. These scalar factors come from the terms $\left(\left(w_{0}^{\prime}\right)^{-c_{1}}\left(w_{1}^{\prime}\right)^{c_{0}}\right)^{(N-1) / 2}$ in $(2-12)$, and the relation $w_{0}^{\prime} w_{1}^{\prime} w_{2}^{\prime}=-\zeta^{-*_{b}(m+1)}$. They are described in the following table, where $v=\exp (\sqrt{-1} \pi(1-N) / 2 N)=\left(-\zeta^{m+1}\right)^{(N-1) / 2}$ and the tetrahedron $\Delta^{i}$ is opposite to the $i$-th vertex, with charge values $c_{k}^{i}$ at the edges:

\begin{tabular}{||c||c|c||c|c|c||}
\hline & $\Delta^{1}$ & $\Delta^{3}$ & $\Delta^{0}$ & $\Delta^{2}$ & $\Delta^{4}$ \\
\hline$(01)$ & 0 & $v^{c_{0}^{3}}$ & 0 & $v^{c_{0}^{2}}$ & $v^{c_{0}^{4}}$ \\
\hline$(12)$ & 0 & $v^{c_{1}^{3}}$ & $v^{c_{0}^{0}}$ & 0 & $v^{c_{1}^{4}}$ \\
\hline$(23)$ & $v^{c_{1}^{1}}$ & 0 & $v^{c_{1}^{0}}$ & 0 & $v^{c_{0}^{4}}$ \\
\hline$(34)$ & $v^{c_{0}^{1}}$ & 0 & $v^{c_{0}^{0}}$ & $v^{c_{0}^{2}}$ & 0 \\
\hline
\end{tabular}

The last equation in (2-19) shows that the scalars at both sides of each row are equal. A similar table leading to the same conclusion can be written for $N=1$ [4, p 519]. This concludes the proof.

Distinguished flat/charged $\mathcal{I}$-triangulations Given an arbitrary nonempty and properly embedded tangle $L$ in $Z$, we say that $(T, H)$ and $\mathcal{T}=(T, H, b, w, f, c)$ are distinguished if $H$ is a subcomplex of the 1 -skeleton of $T$ isotopic to $L$ and passing through all the vertices that are manifold points, and containing no singular vertices (we say that $H$ is Hamiltonian).

Definition 2.17 A distinguished $\mathcal{I}$-triangulation $\mathcal{T}=(T, H, b, w, f, c)$ is flat/charged if $f$ is a global flattening, and

$$
C_{\mathcal{T}}(e)= \begin{cases}0 & \text { if } e \subset H \\ 2 & \text { if } e \subset T \backslash(H \cup \partial T) .\end{cases}
$$

In such a case $c$ is called a global charge.

The existence of global flattenings of an arbitrary $\mathcal{I}$-triangulation with empty boundary, and of global charges (such that $C_{\mathcal{T}}(e)=2$ at all edges $e$ ) on any topological ideal triangulation of an oriented 3-manifold whose boundary consists of tori, was proved by Neumann in [24, Section 9; 23, Section 6]. This last result is easily adapted to the existence of global charges on distinguished triangulations $(T, H)$ [3, Theorem 4.7]. 
We have to refine the QHG isomorphisms in order to deal with distinguished flat/charged $\mathcal{I}$-triangulations. First we have to incorporate the Hamiltonian tangles into the bare moves. Any positive $2 \rightarrow 3$ move $T \rightarrow T^{\prime}$ naturally specializes to a move $(T, H) \rightarrow$ $\left(T^{\prime}, H^{\prime}\right)$; in fact $H^{\prime}=H$ is still Hamiltonian. For positive bubble moves, we assume that an edge $e$ of $H$ lies in the boundary of the involved 2-simplex $f$; then $e$ lies in the boundary of a unique 2-simplex $f^{\prime}$ of $T^{\prime}$ containing the new vertex of $T^{\prime}$. We define the Hamiltonian subcomplex $H^{\prime}$ of $T^{\prime}$ just by replacing $e$ with the other two edges of $f^{\prime}$. The inverse moves are defined in the same way; in particular, for negative $3 \rightarrow 2$ moves we require that the edge disappearing in $T$ belongs to $T^{\prime} \backslash H^{\prime}$. The $2 \leftrightarrow 3$ QHG transit specializes verbatim. For the bubble transit we just impose that the marked edge $e$ (see Section 2.2) coincides with the above edge of $H$. Thanks to (2-20) and (2-21) we see that distinguished flat/charged $\mathcal{I}$-triangulations are closed under such refined QHG transits.

Remark 2.18 The residue $\bmod (2 \sqrt{-1} \pi N)$ of the classical log-branches of a flattened $\mathcal{I}$-triangulation are equivalently given by $N$-th roots $w^{\prime}(h)$ of the cross-ratio moduli $w(h)$ such that $w^{\prime}\left(e_{1}\right) w^{\prime}\left(e_{2}\right) w^{\prime}\left(e_{3}\right)=1$ at each 3-simplex and $\prod_{h \in \epsilon_{T}^{-1}(e)} w^{\prime}(h)^{*_{b}}=1$ at each edge, with the notation of (2-18). For level $N$ quantum log-branches of distinguished flat/charged $\mathcal{I}$-triangulations this is replaced with

and

$$
\begin{aligned}
w^{\prime}\left(e_{1}\right) w^{\prime}\left(e_{2}\right) w^{\prime}\left(e_{3}\right) & =\exp \left(-*_{b} \sqrt{-1} \pi / N\right) \\
\prod_{h \in \epsilon_{T}^{-1}(e)} w^{\prime}(h)^{*} b & = \begin{cases}1 & \text { if } e \subset H \\
\exp (-2 \sqrt{-1} \pi / N) & \text { otherwise }\end{cases}
\end{aligned}
$$

Cohomological weights and the structure of flat/charges Given an $\mathcal{I}$-triangulation $\mathcal{T}=(T, b, w)$ without boundary, let $T_{0}$ be the boundary of an open cone neighborhood of each 0 -simplex. This is a disjoint union of triangulated closed oriented surfaces, the links of the vertices in $T$. To each flattening $f$ of $\mathcal{T}$ a class $\gamma(f) \in H^{1}\left(\partial T_{0} ; \mathbb{C}\right)$ is associated as follows. Represent any integral 1-homology class $a$ of $\partial T_{0}$ by "normal paths", that is, a disjoint union of oriented simple closed curves transverse to the triangulation and such that no component enters and exits from the same face of a 2simplex. Such a curve selects a vertex for each 2 -simplex. The value $\gamma(f)(a)$ is defined as the signed sum of the log-branches of the edges ending at the vertices selected by $a$. For each vertex $v$ the sign is $*_{b}$ if the path goes in the direction given by the orientation of $\partial T_{0}$ as viewed from $v$, and $-*_{b}$ otherwise. Using the flattening condition $\mathrm{F}$ in Section 2.1 and the edge compatibility relations of Definition 2.10 it is easily checked that $\gamma(f)(a)$ does not depend on the choice of normal path representative, and maps unessential loops to zero. Hence $\gamma(f)$ assigns a complex-valued "winding number" to 
integral 1-homology classes on $\partial T_{0}$, and eventually defines a cohomology class in $H^{1}\left(\partial T_{0} ; \mathbb{C}\right)$. Similarly we can define $\gamma_{2}(f) \in H^{1}\left(T_{0} ; \mathbb{Z} / 2 \mathbb{Z}\right)$ by using normal paths in $T_{0}$ and taking modulo 2 sum of the flattenings we meet along the paths. Assume now that $\partial T_{0}$ is a union of tori. By replacing the log-branches with the charges and setting $*_{b}=1$, the same procedure defines an integer-valued map $\gamma(c)$ on the set of essential oriented simple normal loops. Let us extend $\gamma(c)$ by mapping every unessential normal loop to 0 (in spite of the fact that the procedure itself would give the value \pm 2 on such a small loop about a vertex of $\partial T_{0}$ ). Because of the charge condition $C$ in Section 2.1 and the edge compatibility relations (2-5), we can prove that this eventually defines a class $\gamma(c) \in H^{1}\left(\partial T_{0} ; \mathbb{Z}\right)$. By replacing the flattenings with the charges we can also define $\gamma_{2}(c) \in H^{1}\left(T_{0} ; \mathbb{Z} / 2 \mathbb{Z}\right)$.

Definition 2.19 (Weights) The pairs $\left(\gamma(f), \gamma_{2}(f)\right) \in H^{1}\left(\partial T_{0} ; \mathbb{C}\right) \times H^{1}\left(T_{0} ; \mathbb{Z} / 2 \mathbb{Z}\right)$ and $\left(\gamma(c), \gamma_{2}(c)\right) \in H^{1}\left(\partial T_{0} ; \mathbb{Z}\right) \times H^{1}\left(T_{0} ; \mathbb{Z} / 2 \mathbb{Z}\right)$ are called the (cohomological) weights of $f$ and $c$, respectively.

In [23, Section 4] (and [24, Section 9]), Neumann defines an integral chain complex $\mathcal{J}$ from which the global charges are derived. The maps $\gamma$ and $\gamma_{2}$ above are well-defined on the third homology group $H_{3}(\mathcal{J})$ and satisfy:

Theorem 2.20 [23, Theorem 5.1] The sequence

$$
0 \rightarrow H_{3}(\mathcal{J}) \stackrel{\left(\gamma_{2}, \gamma\right)}{\longrightarrow} H^{1}\left(T_{0} ; \mathbb{Z} / 2 \mathbb{Z}\right) \oplus H^{1}\left(\partial T_{0} ; \mathbb{Z}\right) \stackrel{i^{*}-r}{\longrightarrow} H^{1}\left(\partial T_{0} ; \mathbb{Z} / 2 \mathbb{Z}\right) \rightarrow 0
$$

is exact, where $r: H^{1}\left(\partial T_{0} ; \mathbb{Z}\right) \rightarrow H^{1}\left(\partial T_{0} ; \mathbb{Z} / 2 \mathbb{Z}\right)$ is the coefficient map and the map $i^{*}: H^{1}\left(T_{0} ; \mathbb{Z} / 2 \mathbb{Z}\right) \rightarrow H^{1}\left(\partial T_{0} ; \mathbb{Z} / 2 \mathbb{Z}\right)$ is induced by the inclusion $\partial T_{0} \rightarrow T_{0}$.

It is easy to check that the weights $\left(h_{c}, k_{c}\right)=\left(\gamma_{2}(c), \gamma(c)\right)$ of a global charge $c$ satisfy $r\left(k_{c}\right)=i^{*}\left(h_{c}\right)$. Conversely, by using Theorem 2.20 it can be shown that for any pair $(h, k) \in H^{1}\left(T_{0} ; \mathbb{Z} / 2 \mathbb{Z}\right) \times H^{1}\left(\partial T_{0} ; \mathbb{Z}\right)$ such that $r(k)=i^{*}(h)$ there exist global charges $c$ such that $\left(h_{c}, k_{c}\right)=(h, k)$ (see Neumann [23, proof of Lemma 6.1]). This fact extends to log-branches as follows. Let $\gamma^{\prime}(a)$ be defined as $\gamma(f)(a)$ above, except that for each 3 -simplex the log-branches are replaced with the natural logs of the cross ratios. In fact $\gamma^{\prime} \in H^{1}\left(\partial T_{0} ; \mathbb{C} / 2 \sqrt{-1} \pi \mathbb{Z}\right)$. It can be checked that $\gamma^{\prime}(a)$ is the logarithm of the derivative of the holonomy of $a$ (a similarity), up to multiples of $2 \sqrt{-1} \pi$. Then, any pair $(h, k)$ in the product $H^{1}\left(T_{0} ; \mathbb{Z} / 2 \mathbb{Z}\right) \times H^{1}\left(\partial T_{0} ; \mathbb{C}\right)$ such that

$$
\begin{cases}k(a)=\gamma^{\prime}(a) \bmod (\sqrt{-1} \pi), & \text { for all } a \in H_{1}\left(\partial T_{0} ; \mathbb{Z}\right) \\ \left(k(a)-\gamma^{\prime}(a)\right) / \sqrt{-1} \pi=i^{*}(h)(a) \bmod (2), & \text { for all } a \in H_{1}\left(\partial T_{0} ; \mathbb{Z}\right)\end{cases}
$$


is a weight for some flattening. For instance, when $\gamma^{\prime}(a) \in 2 \sqrt{-1} \pi \mathbb{Z}$ for all $a$ the first condition means that $k$ is integral.

Finally, the structure of the spaces of flattenings and integral charges is given by Theorem 2.4 in [23]. For each fixed weight $(h, k)$ they form an affine space over an integral lattice. Generators have the following combinatorial realization: for each 3 -simplex in the star of an edge $e$, add +1 to the flat/charges of one of the two other pairs of opposite edges, and -1 for the other pair, so that the total log-branches or charges stay equal everywhere. In particular, for flattenings of an idealization $\mathcal{I}_{\mathcal{I}}$ any generator is obtained by adding +1 to the log determination at some edge. Hence any flattening of $\mathcal{T}_{\mathcal{I}}$ inducing the weight of the canonical flattening of Lemma 2.13 differs from it as described in the statement.

We note that the above refined QHG isomorphisms preserve the weights; see eg [3, Lemma 4.12]. The difference in considering global flattenings or charges with different $\bmod (2)$ weights in $H^{1}\left(T_{0} ; \mathbb{Z} / 2 \mathbb{Z}\right)$ seems to carry not so essential information; see Theorem 5.4 and Remark 5.6. This contrasts with boundary weights in $H^{1}\left(\partial T_{0} ; \mathbb{C}\right)$, which play a key role in surgery formulae; see [24, Theorems 14.5 and 14.7] and Section 6.2. We note that a process involving 2-handle surgery allows to define explicit isomorphisms between lattices of flat/charges with different boundary weights [24, Section 11, p 457].

\section{Parameters for $\operatorname{PSL}(2, \mathbb{C})$-characters of surfaces}

Fix a compact closed oriented surface $S$ of genus $g$ with a nonempty set $V=$ $\left\{v_{1}, \ldots, v_{r}\right\}$ of marked points, and negative Euler characteristic $\chi(S \backslash V)<0$. Denote by $\pi$ the fundamental group of $S \backslash V$, and by

$$
\mathcal{R}(g, r)=\operatorname{Hom}(\pi, \operatorname{PSL}(2, \mathbb{C})) / P S L(2, \mathbb{C})
$$

the set of all conjugacy classes of $P S L(2, \mathbb{C})$-valued representations of $\pi$. The group $\pi$ is free of rank $\kappa=2 g+r-1$. Any choice of free generators of $\pi$ identifies the set $\operatorname{Hom}(\pi, P S L(2, \mathbb{C}))$ with $\operatorname{PSL}(2, \mathbb{C})^{\kappa}$. Different such identifications are related by algebraic automorphisms of $\operatorname{PSL}(2, \mathbb{C})^{\kappa}$. Moreover, the isomorphism $P S L(2, \mathbb{C}) \cong S O(3, \mathbb{C})$ induced by the adjoint action Ad: $P S L(2, \mathbb{C}) \rightarrow \operatorname{Aut}(\operatorname{sl}(2, \mathbb{C}))$ implies that $\operatorname{Hom}(\pi, P S L(2, \mathbb{C}))$ is an affine complex algebraic set, with the complex algebraic action of $P S L(2, \mathbb{C})$ by conjugation.

As the rough topological quotient space $\mathcal{R}(g, r)$ is not Hausdorff, it is more convenient to consider the algebro-geometric quotient

$$
X(\pi)=\operatorname{Hom}(\pi, \operatorname{PSL}(2, \mathbb{C})) / / P S L(2, \mathbb{C})
$$


of invariant theory, called the variety of $\operatorname{PSL}(2, \mathbb{C})$-characters (see eg Heusener and Porti [19]). Recall that $X(\pi)$ is a complex affine algebraic set such that there exists a surjective regular map

$$
t: \operatorname{Hom}(\pi, P S L(2, \mathbb{C})) \rightarrow X(\pi)
$$

inducing an isomorphism $t^{*}$ between the regular functions on $X(\pi)$ and the regular functions on $\operatorname{Hom}(\pi, P S L(2, \mathbb{C}))$ invariant by conjugation. In general $t(\gamma)=t(\sigma)$ does not imply that $\gamma$ and $\sigma$ are conjugate, but this is true if we restrict to irreducible representations: we have $\operatorname{Hom}^{\mathrm{irr}}(\pi, P S L(2, \mathbb{C}))=t^{-1}\left(X^{\mathrm{irr}}(\pi)\right)$, where $X^{\mathrm{irr}}(\pi)=$ $t\left(\operatorname{Hom}^{\mathrm{irr}}(\pi, P S L(2, \mathbb{C}))\right.$, so that the (restricted) rough quotient $\mathcal{R}(g, r)^{\mathrm{irr}}$ and the algebraic quotient $X^{\mathrm{irr}}(\pi)$ coincide.

We can deal with the whole of $\mathcal{R}(g, r)$ to construct the QHFT. Hence below we treat the complex dimension of subsets of $\mathcal{R}(g, r)$ somewhat formally, as everything can be substantiated in terms of $X(\pi)$, or by restriction to $X^{\text {irr }}(\pi)$. For instance, as $\operatorname{PSL}(2, \mathbb{C})$ has trivial center and complex dimension 3 we say that the complex dimension of $\mathcal{R}(g, r)$ is $3 \kappa-3=-3 \chi(F)$.

\subsection{Efficient triangulations}

Fix a surface $F$ with $r$ boundary components, obtained by removing from $S$ the interior of small 2-disks $D_{i}$ such that $v_{i} \in \partial D_{i}$. The boundary of $F$ is oriented via the convention: last is the ingoing normal.

A triangulation $T^{\prime}$ of $S$ with the set of vertices equal to $V$ is called a topological ideal triangulation of $S \backslash V$. Given such a $T^{\prime}$, we need a marking of corners of the 2-simplices. The best suited for 3-dimensional extension are induced by global branchings $b^{\prime}$ of $T^{\prime}$, and it is known that pairs $\left(T^{\prime}, b^{\prime}\right)$ always exist. Then, as in Section 2, we have a sign function $\sigma=\sigma_{\left(T^{\prime}, b^{\prime}\right)}$. A corner map $v \mapsto c_{v}$ associates to each vertex $v$ of $T^{\prime}$ the corner at $v$ of a triangle, say $t_{v}$, in its star. We say that $v \mapsto c_{v}$ is $t$-injective if $v \mapsto t_{v}$ is injective.

Lemma 3.1 For every $(g, r) \neq(0,3)$, every triangulation $T^{\prime}$ of $S$ with $r$ vertices admits $t$-injective corner maps.

Proof For $(g, r)=(0,4)$ or $g>0$ and $r=1$, it is immediate to construct such a triangulation. Subdividing a triangle by taking the cone from an interior point preserves the existence of $t$-injective corner maps. By induction on $r$ we deduce that for every $(g, r) \neq(0,3)$ there exist triangulations of $S$ as in the statement.

In Figure 6 the corner selection is specified by a $*$, and the rows show all the possible flip moves on triangulations of $S$, up to obvious symmetries, that preserve the injectivity 
of corner maps. Consider triangulations $T^{\prime}, T^{\prime \prime}$ of $S$ with $r$ vertices, such that $T^{\prime \prime}$ supports a $t$-injective corner map. It is well known that $T^{\prime \prime}$ is connected to $T^{\prime}$ via a finite sequence of flips. The $t$-injective corner map for $T^{\prime \prime}$ yields a $t$-injective corner map for $T^{\prime}$ by decorating these flips as in Figure 6.
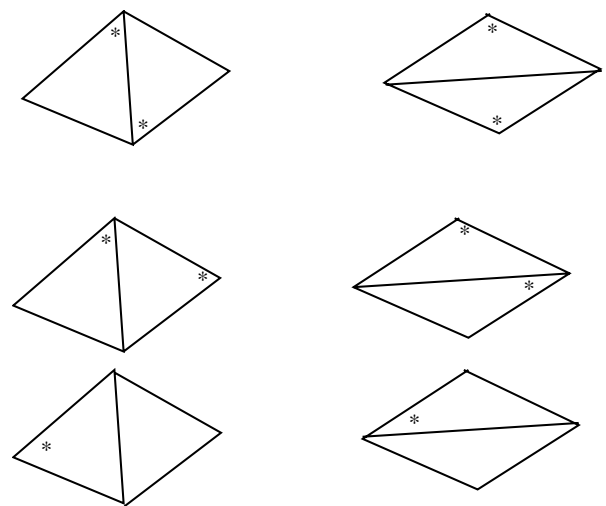

Figure 6: The flips with marked corners

There are no obstructions to use arbitrary corner maps in what follows. Specializing to injective ones just simplifies the exposition. For $(g, r)=(0,3)$, triangulations have two 2-simplices, one with two selected corners. From now on, we assume that $(g, r) \neq(0,3)$, the extension to the $(0,3)$ case being straightforward.

Given a pair $(g, r) \neq(0,3)$ and $\left(T^{\prime}, b^{\prime}\right)$ as in Lemma 3.1, fix a $t$-injective corner map $v \mapsto c_{v}$. In the interior of each triangle $t_{v}$ consider a bigon $D_{v}$ with one vertex at $v$, and call $v^{\prime}$ the other vertex. Remove from $t_{v}$ the interior of $D_{v}$, and triangulate the resulting cell $s_{v}=t_{v} \backslash \operatorname{Int}\left(D_{v}\right)$ by taking the cone with base $v^{\prime}$. Repeating this procedure for each $t_{v}$, we get a triangulation $T$ of $F$ with $2 r$ vertices and $p+2 r$ triangles, where $p$ denotes the number of triangles of $T^{\prime}$. The set of edges of $T$, $E(T)$, contains $E\left(T^{\prime}\right)$ in a natural way, and $|E(T)|=\left|E\left(T^{\prime}\right)\right|+4 r$. We extend $b^{\prime}$ to a branching $b$ on $T$ as in Figure 7, and the sign function $\sigma_{\left(T^{\prime}, b^{\prime}\right)}$ to the triangles of $(T, b)$ in the natural way. Note that the figure shows only one of the possible branching configurations. In general we extend $b^{\prime}$ to $b$ so that we can recover $\left(T^{\prime}, b^{\prime}\right)$ from $(T, b)$ by "zipping" and "collapsing", as suggested at the bottom of Figure 7 . In what follows, for simplicity we will refer to this configuration, as the treatment of the others is similar.

Definition 3.2 We call a pair $(T, b)$ constructed as above from a pair $\left(T^{\prime}, b^{\prime}\right)$ an efficient triangulation (e-triangulation for short) of $F$. For each vertex $v$, the preferred 


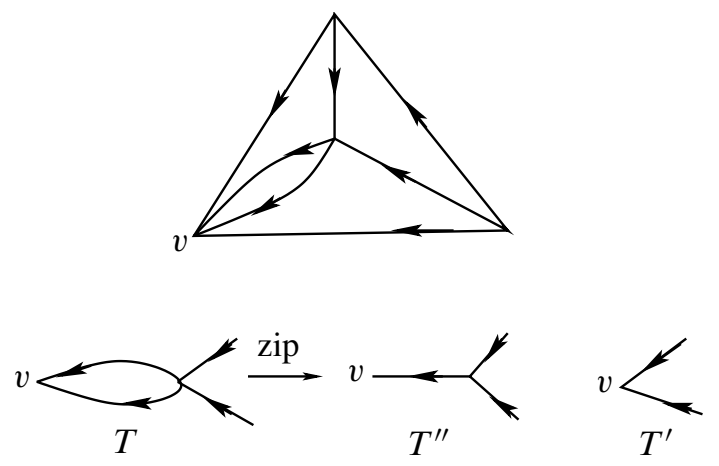

Figure 7: The branched triangulated cell $s_{v}$

inner triangle at $v$ is the triangle $\tau_{v}$ in $s_{v}$ with an edge on $\partial F$ whose $b$-orientation coincides with the boundary orientation of $F$.

\subsection{Cocycle parameters}

The inclusions of $\operatorname{Int}(F)$ into $F$ and $S \backslash V$ induce identifications of the respective fundamental groups.

Fix an $e$-triangulation $(T, b)$ of $F$. Denote by $Z(T, b)$ the space of $P S L(2, \mathbb{C})-$ valued 1 -cocycles on $(T, b)$; we stipulate that on a triangle with ordered $b$-oriented edges $e_{0}, e_{1}, e_{2}$ the cocycle relation is $z\left(e_{0}\right) z\left(e_{1}\right) z\left(e_{2}\right)^{-1}=1$. In this section we construct a parametrization of $\mathcal{R}(g, r)$ based on cocycle coefficients by specifying subsets of $Z(T, b)$ with small "residual gauge groups", that make principal algebraic bundles over the "strata" of a suitable partition of $\mathcal{R}(g, r)$. These strata are determined by the holonomies around the boundary components of $F$.

Write $C(T, b)$ for the space of $\operatorname{PSL}(2, \mathbb{C})$-valued 0 -cochains on $(T, b)$, that is the $\operatorname{PSL}(2, \mathbb{C})$-valued functions defined on the set of vertices of $T$. Two 1-cocycles $z$ and $z^{\prime}$ are said equivalent up to gauge transformation if there is a 0 -cochain $\lambda$ such that, for every oriented edge $e=\left[x_{0}, x_{1}\right]$, we have

$$
z^{\prime}(e)=\lambda\left(x_{0}\right)^{-1} z(e) \lambda\left(x_{1}\right) .
$$

It is well known that the quotient set $H(T, b)=Z(T, b) / C(T, b)$ is in one-one correspondence with $\mathcal{R}(g, r)$. Indeed, for any fixed $x_{0} \in T$ we have a natural surjective map

$$
f_{x_{0}}: Z(T, b) \rightarrow \operatorname{Hom}(\pi, P S L(2, \mathbb{C})),
$$


where $\pi=\pi_{1}\left(F, x_{0}\right)$, and two representations $f_{x_{0}}(z)$ and $f_{x_{0}}\left(z^{\prime}\right)$ define the same point in $\mathcal{R}(g, r)$ if and only if $z$ and $z^{\prime}$ are equivalent up to gauge transformation. Note that the complex dimension of $H(T, b)$ is $3(|E(T)|-(p+2 r)-2 r)=-3 \chi(F)$, which is the dimension of $\mathcal{R}(g, r)$. This is because the complex dimension of $C(T, b)$ is equal to $6 r$, the set $Z(T, b)$ is defined by $3(p+2 r)$ polynomial relations on $3|E(T)|$ variables, and $\operatorname{PSL}(2, \mathbb{C})$ has trivial center. (Recall that $p$ is the number of triangles of the initial triangulation $T^{\prime}$ of $S$ with $r$ vertices.)

Denote by $B^{+}(2, \mathbb{C})$ (respectively $B^{-}(2, \mathbb{C})$ ) the Borel subgroup of $S L(2, \mathbb{C})$ of upper (respectively lower) triangular matrices. Let $P B^{ \pm}(2, \mathbb{C})=B^{ \pm}(2, \mathbb{C}) / \pm I$ and put

$$
P=\left(\begin{array}{ll}
0 & 1 \\
1 & 0
\end{array}\right)
$$

Define a map

$$
\Psi: S L(2, \mathbb{C}) \rightarrow S L(2, \mathbb{C}), \quad \Psi(A)=P A P,
$$

and denote by $\Psi$ the induced automorphism of $\operatorname{PSL}(2, \mathbb{C})$. We have $\Psi\left(B^{ \pm}(2, \mathbb{C})\right)=$ $B^{\mp}(2, \mathbb{C})$.

For any $g \in P S L(2, \mathbb{C})$ we distinguish the type of $g$ as trivial, parabolic or generic (the latter for elliptic or loxodromic) with the obvious meaning. We denote by

$$
\mathcal{C}^{+}(g) \in P B^{+}(2, \mathbb{C})
$$

the canonical upper triangular matrix (up to sign) representative of the conjugacy class of $g$ (for generic $g$ we normalize $\mathcal{C}^{+}(g)$ by stipulating that the top diagonal entry has absolute value $>1$ ), and we set

$$
\mathcal{C}^{-}(g)=\Psi\left(\mathcal{C}^{+}(g)\right)
$$

We define the type of $\rho \in \mathcal{R}(g, r)$ as the $r$-tuple of types of the $\rho$-holonomies of the oriented boundary components $\gamma_{1}, \ldots, \gamma_{r}$ of $F$. Put

$$
\begin{aligned}
\mathcal{R}(g, r, t) & =\{\rho \in \mathcal{R}(g, r) \mid \rho \text { has type } t\} \\
\mathcal{R}\left(g, r, \mathcal{C}^{ \pm}\right) & =\left\{\rho \in \mathcal{R}(g, r, t) \mid \mathcal{C}^{ \pm}\left(\rho\left(\gamma_{i}\right)\right)=\mathcal{C}_{i}^{ \pm}\right\}
\end{aligned}
$$

where $\mathcal{C}^{ \pm}=\left(\mathcal{C}_{1}^{ \pm}, \ldots, \mathcal{C}_{r}^{ \pm}\right), \mathcal{C}_{i}^{ \pm}$is an arbitrary diagonal or unipotent element in $P B^{ \pm}(2, \mathbb{C})$, and $\mathcal{C}^{ \pm}$has type $t$.

For any $g \in P B^{ \pm}(2, \mathbb{C})$ let us write $g=[a, b]^{ \pm}$, where $a$ is the top diagonal entry of $g$ and $b$ is the nondiagonal one (we do the abuse of confusing $g$ with its projective class). For every vertex $v_{i}$ of $(T, b)$, let $e_{1}^{i}$ be the boundary edge of the preferred inner 
triangle $\tau_{v_{i}}$ (see Definition 3.2), and $\gamma_{i}$ the oriented boundary loop of $F$ based at $v_{i}$. Recall the projections $f_{v_{i}}: Z(T, b) \rightarrow \operatorname{Hom}(\pi, P S L(2, \mathbb{C}))$.

Definition 3.3 The set of $( \pm)$-cocycle parameters for $\mathcal{R}\left(g, r, \mathcal{C}^{ \pm}\right)$is $\mathcal{Z}\left(T, b, \mathcal{C}^{ \pm}\right)=\left\{z \in Z(T, b) \mid\right.$ for $\left.i=1, \ldots, r, f_{v_{i}}(z)\left(\gamma_{i}\right)=\mathcal{C}_{i}^{ \pm}, z\left(e_{1}^{i}\right)=[1,1 / 2]^{ \pm}\right\}$.

Clearly $\mathcal{Z}\left(T, b, \mathcal{C}^{ \pm}\right)$is nonempty. In fact, any $z \in Z(T, b)$ such that conj $\circ f(z) \in$ $\mathcal{R}\left(g, r, \mathcal{C}^{ \pm}\right)$is equivalent to one in $\mathcal{Z}\left(T, b, \mathcal{C}^{ \pm}\right)$via some gauge transformation. Moreover, given a vertex $v_{i}$ and a 0 -cochain $s$ with support at $v_{i}$ and $v_{i}^{\prime}, s$ maps $\mathcal{Z}\left(T, b, \mathcal{C}^{ \pm}\right)$ onto itself if and only if

and

$$
\begin{gathered}
s\left(v_{i}\right) \in \operatorname{Stab}\left(\mathcal{C}_{i}^{ \pm}\right) \\
s\left(v_{i}^{\prime}\right)^{-1}[1,1 / 2]^{ \pm} s\left(v_{i}\right)=[1,1 / 2]^{ \pm} .
\end{gathered}
$$

As the triangles $\tau_{v_{i}}$ are in one-one correspondence with the $v_{i}$, we deduce that there is a projection

$$
p_{\mathcal{C}}^{ \pm}: \mathcal{Z}\left(T, b, \mathcal{C}^{ \pm}\right) \rightarrow \mathcal{R}\left(g, r, \mathcal{C}^{ \pm}\right)
$$

with fiber isomorphic to the group

$$
\mathcal{G}\left(T, b, \mathcal{C}^{ \pm}\right):=\operatorname{Stab}\left(\mathcal{C}_{1}^{ \pm}\right) \times \cdots \times \operatorname{Stab}\left(\mathcal{C}_{r}^{ \pm}\right)
$$

that we call the residual gauge transformations. Denote by $\mathcal{Z}(T, b, t)^{ \pm}$(respectively $p_{t}^{ \pm}$) the union of the $\mathcal{Z}\left(T, b, \mathcal{C}^{ \pm}\right)$(respectively $p_{\mathcal{C}}^{ \pm}$) over all $\mathcal{C}^{ \pm}$with type $t$, and put

$$
\mathcal{Z}(T, b)^{ \pm}=\bigsqcup_{t} \mathcal{Z}(T, b, t)^{ \pm}, \quad p^{ \pm}=\bigsqcup_{t} p_{t}^{ \pm} .
$$

For future reference, we summarize the above constructions in the following proposition.

Proposition 3.4 For every $\mathcal{C}^{ \pm}$, the projection $p^{ \pm}: \mathcal{Z}(T, b)^{ \pm} \rightarrow \mathcal{R}(g, r)$ restricts to a complex affine algebraic principal $\mathcal{G}\left(T, b, \mathcal{C}^{ \pm}\right)$-bundle

$$
\mathcal{Z}\left(T, b, \mathcal{C}^{ \pm}\right) \rightarrow \mathcal{R}\left(g, r, \mathcal{C}^{ \pm}\right)
$$

The map $\Psi$ in (3-1) yields an isomorphism $\mathcal{Z}\left(T, b, \mathcal{C}^{ \pm}\right) \cong \mathcal{Z}\left(T, b, \mathcal{C}^{\mp}\right)$. Moreover we have

$$
\begin{aligned}
\operatorname{dim}\left(\mathcal{Z}\left(T, b, \mathcal{C}^{ \pm}\right)\right. & =-3 \chi(F) \\
\operatorname{dim}\left(\mathcal{R}\left(g, r, \mathcal{C}^{ \pm}\right)\right) & =-3 \chi(F)-\operatorname{dim}\left(\mathcal{G}\left(T, b, t_{\mathcal{C}}\right)\right) \\
\operatorname{dim}\left(\mathcal{R}\left(g, r, t_{\mathcal{C}}\right)\right) & =\operatorname{dim}\left(\mathcal{R}\left(g, r, \mathcal{C}^{ \pm}\right)\right)+\alpha\left(t_{\mathcal{C}}\right)
\end{aligned}
$$

where $t_{\mathcal{C}}$ is the type of $\mathcal{C}^{ \pm}$and $\alpha\left(t_{\mathcal{C}}\right)$ is the number of generic entries of $t_{\mathcal{C}}$. 
Observe that if $t_{\text {gen }}$ is the purely generic type, then $\operatorname{dim}\left(\mathcal{R}\left(g, r, t_{\text {gen }}\right)\right)=-3 \chi(F)$; if $t_{\text {par }}$ is purely parabolic, then $\operatorname{dim}\left(\mathcal{R}\left(g, r, t_{\mathrm{par}}\right)\right)=-3 \chi(F)-r$; if $t_{I}$ is purely trivial, then $\operatorname{dim}\left(\mathcal{R}\left(g, r, t_{I}\right)\right)=-3 \chi(F)-3 r=-3 \chi(S)=6 g-6$. Hence $\mathcal{R}\left(g, r, t_{\text {gen }}\right)$ is a dense open subset of $\mathcal{R}(g, r)$. Moreover, via the inclusion of closures, we have a filtration of $\mathcal{R}(g, r)$ for which $\mathcal{R}\left(g, r, t_{I}\right)$ is the "deepest" part. It would be interesting to study the singularities of the closure of each $\mathcal{R}(g, r, t)$ in $\mathcal{R}(g, r)$, in order to check if this filtration induces a "stratification" of $\mathcal{R}(g, r)$.

Remark 3.5 For parabolic elements $g \in P S L(2, \mathbb{R})$ we have two conjugacy classes, that can be distinguished by a sign. So, replacing $\operatorname{PSL}(2, \mathbb{C})$ with $\operatorname{PSL}(2, \mathbb{R})$, the constructions of this section still work by associating a sign to each parabolic end of the surface $S \backslash V$. Note that for $P S L(2, \mathbb{R})$-valued cocycles the idealization procedure described in Section 3.3 below gives only degenerate tetrahedra (with real cross-ratio moduli).

Example 3.6 (The Fricke space) Suppose that $S$ has a unique marked point $v$. Choose a standard curve system $\mathcal{S}=\left\{a_{i}, b_{i}\right\}_{i=1}^{g}$ based at $v$, so that

$$
\pi_{1}(S, v)=\left\langle a_{1}, b_{1}, \ldots, a_{g}, b_{g} \mid\left[a_{1}, b_{1}\right] \ldots\left[a_{g}, b_{g}\right]=1\right\rangle .
$$

Cutting open $S$ along $\mathcal{S}$ we get a $4 g$-gon $P$ with oriented boundary edges. Taking the cone to a vertex it is easy to construct a branched triangulation of $P$, which induces one, say $\left(T^{\prime}, b^{\prime}\right)$, for $(S, v)$. Denote by $(T, b)$ any $e$-triangulation obtained from $\left(T^{\prime}, b^{\prime}\right)$.

Recall that the Teichmüller space $\mathcal{T}(S)$ can be identified with the set of conjugacy classes of $P S L(2, \mathbb{R})$-valued discrete faithful representations of $\pi_{1}(S)$, and that we have the well-known (real-analytic) Fricke parametrization $\mathcal{T}(S) \cong \mathbb{R}^{6 g-6}$ (see eg Abikoff [1]). For each $z \in \mathcal{T}(S)$ the Fricke coordinates of $z$ are matrix entries of the $\widehat{z}(\gamma)$ for all $\gamma \in \mathcal{S}$, where $\widehat{z}$ is a representative of $z$ specified by fixing once and for all three of the fixed points of $z\left(a_{g}\right)$ and $z\left(b_{g}\right)$. So $\mathcal{T}(S)$ embeds in the space of real cocycle parameters for $F$ with trivial type $t_{I}$. This embedding is generalized easily to the Teichmüller space of arbitrary bordered Riemann surfaces, by considering the spaces $\mathcal{Z}(T, b, t)$ for all types $t$ and $e$-triangulations of $(S, V)$ with arbitrary $V$ (see Remark 3.5).

\subsection{Cross-ratio parameters}

In this section we derive from the cocycle parameters $\mathcal{Z}(T, b)^{ \pm}$other parameters for $\mathcal{R}(g, r)$ which are related to the exponential shear-bend coordinates for pleated hyperbolic surfaces. These parameters are obtained via an idealization procedure that 
includes the choice of a base point on the Riemann sphere. We fix this base point as 0 . Note that the $B^{+}(2, \mathbb{C})$-orbit of 0 is the whole of $\mathbb{C}$, while $B^{-}(2, \mathbb{C})$ fixes 0 . Hence the symmetry between $\mathcal{Z}(T, b)^{+}$and $\mathcal{Z}(T, b)^{-}$given by the map $\Psi$ in (3-1) shall be broken.

Definition 3.7 (Compare with Definition 2.11.) Let $(K, b)$ be any oriented surface branched triangulation. Let $z$ be any $\operatorname{PSL}(2, \mathbb{C})$-valued cocycle on $(K, b)$. We say that $z$ is idealizable if for any triangle $t$ of $(T, b)$ with $b$-ordered edges $e_{0}, e_{1}$ and $e_{2}$, the points $u_{0}=0, u_{1}=z\left(e_{0}\right)(0)$ and $u_{2}=z\left(e_{2}\right)(0)$ are distinct in $\mathbb{C}$. We say that the complex triangle with vertices $u_{0}, u_{1}$ and $u_{2}$ is the idealization of $t$.

Because we use the cocycle rule corresponding to the multiplication from the right in $\operatorname{PSL}(2, \mathbb{C})$, the property of a cocycle to be idealizable does not depend on the branching.

Definition 3.8 Let $(T, b)$ be an $e$-triangulation of $F$ obtained from a branched ideal triangulation $\left(T^{\prime}, b^{\prime}\right)$ of $S \backslash V$. A cocycle $z \in \mathcal{Z}(T, b)^{+}$is strongly idealizable if:

(a) $z$ is idealizable.

(b) $\Psi(z) \in \mathcal{Z}(T, b)^{-}$, which cannot be idealizable at the triangles having an edge on $\partial F$, is nevertheless idealizable at every other triangle.

Denote by $\mathcal{Z}_{I}(T, b)^{+}$the set of strongly idealizable cocycles, and put $\mathcal{Z}_{I}(T, b)^{-}=$ $\Psi\left(\mathcal{Z}_{I}(T, b)^{+}\right)$and $\mathcal{R}_{I}(T, b)=p^{+}\left(\mathcal{Z}_{I}(T, b)^{+}\right)=p^{-}\left(\mathcal{Z}_{I}(T, b)^{-}\right)$.

Clearly, $\mathcal{Z}_{I}(T, b)^{+}$is a nonempty dense open subset of $\mathcal{Z}(T, b)^{+}$. If every edge of $T$ has distinct endpoints (in which case we say that $T$ is quasi-regular), then $\mathcal{R}_{I}(T, b)=\mathcal{R}(g, r)$. In general, characters of representations with a free action on a nonempty domain of $\mathbb{C P}^{1}$ (such as quasi-Fuchsian representations) always belong to $\mathcal{R}_{I}(T, b)$, for any $(T, b)$. By using the arguments of Kashaev [21, Theorem 1], it can be shown that for any character $\rho \in \mathcal{R}(g, r)$ of irreducible representations, there exists an $e$-triangulation $(T, b)$ with idealizable cocycles representing $\rho$. In fact, the union of a finite number of spaces $\mathcal{Z}_{I}(T, b)^{+}$cover the whole of $\mathcal{R}(g, r)^{\mathrm{irr}}$.

(+)-Exponential $\mathcal{I}$-parameters For any strongly idealizable cocycle $z \in \mathcal{Z}_{I}(T, b)^{+}$ we associate a nonzero complex number $W^{+}(z)(e)$ to each edge $e$ of $T$ that is not contained in $\partial F$, as follows. Let $p_{e}$ be the initial endpoint of $e$, and $t_{l}$ and $t_{r}$ the left and right adjacent triangles (as viewed from $e$ ). Locally modify the branching on $t_{l} \cup t_{r}$ by cyclically reordering the vertices on each triangle, so that $p_{e}$ is eventually the source of the new branching on both $t_{l}$ and $t_{r}$. The $(+)$-exponential $\mathcal{I}$-parameter $W^{+}(z)(e)$ 
is the cross-ratio modulus at $e$ of the (possibly degenerate) branched oriented hyperbolic ideal tetrahedron spanned by the idealization of $t_{r} \cup t_{l}$, where the branching completes the one of $t_{l} \cup t_{r}$ so that $*_{b}=1$.

(-)-Exponential $\mathcal{I}$-parameters For each $v \in V$, denote by $e_{v}^{1}$ and $e_{v}^{2}$ the edges of the triangle $t_{v}$ of $T^{\prime}$ having $c_{v}$ as corner. At an edge $e$ of $T^{\prime}$ distinct from any of the $e_{v}^{i}$, we define the (-)-exponential $\mathcal{I}$-parameter $W^{-}(z)(e)$ in the same way as $W^{+}(z)(e)$, but taking the idealization for $\Psi(z) \in \mathcal{Z}_{I}(T, b)^{-}$instead of $z$. If $e$ is one of the $e_{v}^{i}$, the formula works as well, except that each left/right triangle with an edge on $\partial F$ is replaced with the innermost triangle in the corresponding triangle $t_{v}$ of $T^{\prime}$. Again $t_{r} \cup t_{l}$ is a quadrilateral, because 0 is a fixed point of the cocycle values at the boundary edges of $F$.

By varying the edge in $T$ or $T^{\prime}$, for every tuple $\mathcal{C}^{ \pm}$of conjugacy class representatives of the boundary holonomies these constructions give us two $( \pm)$-exponential $\mathcal{I}$ parameter maps (recall that the number of edges of $T^{\prime}$ is $-3 \chi(F)$, and $p$ denotes the number of triangles of $\left.T^{\prime}\right)$ :

$$
\begin{aligned}
& W^{+}: \mathcal{Z}_{I}\left(T, b, \mathcal{C}^{+}\right) \longrightarrow(\mathbb{C} \backslash\{0\})^{-3 \chi(F)+2 p} \\
& W^{-}: \mathcal{Z}_{I}\left(T, b, \mathcal{C}^{-}\right) \longrightarrow(\mathbb{C} \backslash\{0\})^{-3 \chi(F)} .
\end{aligned}
$$

Definition 3.9 We call $W^{ \pm}(T, b, \mathcal{C})=W^{ \pm}\left(\mathcal{Z}_{I}\left(T, b, \mathcal{C}^{ \pm}\right)\right)$the $( \pm)$-exponential $\mathcal{I}$ parameter space of $\mathcal{R}_{I}(T, b, \mathcal{C})$.

We stress again that the result of the idealization strongly depends on the choice of the base point, here 0 . In particular, the exponential $\mathcal{I}$-parameters are not invariant under arbitrary gauge transformations of cocycles. A remarkable exception is for gauge transformations associated to 0 -cochains $\lambda$ with values in $\mathrm{PB}^{-}(2, \mathbb{C})$. Indeed, these act on the idealization of quadrilaterals as conformal transformations of the four vertices (this is because 0 is fixed by every $\lambda(v), v \in V$ ), and cross-ratios are conformal invariants. This makes a big difference between $W^{+}$and $W^{-}$. In fact the whole of the residual gauge group $\mathcal{G}\left(T, b, \mathcal{C}^{+}\right)$acts on $W^{+}(T, b, \mathcal{C})$ via the map $W^{+}$. On the other hand, consider the subgroup of $\mathcal{G}\left(T, b, \mathcal{C}^{-}\right)$defined as

$$
B \mathcal{G}\left(T, b, \mathcal{C}^{-}\right):=\prod_{i=1, \ldots, r} \operatorname{Stab}\left(\mathcal{C}_{i}^{-}\right) \cap P B^{-}(2, \mathbb{C}) .
$$

Any fiber $\left(W^{-}\right)^{-1}\left(W^{-}(z)\right)$ is given by the $B \mathcal{G}\left(T, b, \mathcal{C}^{-}\right)$-orbit of $z$ in $\mathcal{Z}_{I}\left(T, b, \mathcal{C}^{-}\right)$. Hence the actual residual gauge transformations of $W^{-}(T, b, \mathcal{C})$ are in one-one correspondence with the quotient set $\mathcal{G}\left(T, b, \mathcal{C}^{-}\right) / B \mathcal{G}\left(T, b, \mathcal{C}^{-}\right)$, where the equivalence 
class of $\lambda$ is $B \mathcal{G}\left(T, b, \mathcal{C}^{-}\right) \lambda$. For the matter of notational convenience, formally put $B \mathcal{G}\left(T, b, \mathcal{C}^{+}\right):=\prod_{i=1, \ldots, r}$ Id. The map

$$
\begin{aligned}
\Theta^{ \pm}: W^{ \pm}(T, b, \mathcal{C}) & \longrightarrow \mathcal{R}\left(g, r, \mathcal{C}^{ \pm}\right) \\
W^{ \pm}(z) & \longmapsto p_{\mathcal{C}}^{ \pm}(z)
\end{aligned}
$$

with $p_{\mathcal{C}}^{ \pm}$defined in (3-2), is a principal $\mathcal{G}\left(T, b, \mathcal{C}^{ \pm}\right) / B \mathcal{G}\left(T, b, \mathcal{C}^{ \pm}\right)$-bundle. The situation is particularly clean when $\mathcal{C}^{-}$has no trivial entries:

Proposition 3.10 If $\mathcal{C}^{-}$has no trivial entries the (-)-exponential $\mathcal{I}$-parameter map $W^{-}: \mathcal{Z}_{I}\left(T, b, \mathcal{C}^{-}\right) \rightarrow W^{-}(T, b, \mathcal{C})$ is invariant under gauge transformations.

(-)-Exponential $\mathcal{I}$-parameters and pleated surfaces When $\mathcal{C}$ has no trivial entries, there is a nice interpretation of the parameter space $W^{-}(T, b, \mathcal{C})$ in terms of pleated hyperbolic surface structures on $S \backslash V$ (see eg Canary, Epstein and Green [10]; compare also with Bonahon and Liu [9, Section 8]).

As before, let $T^{\prime}$ be a triangulation of $S$ with vertices $V$, viewed as an ideal triangulation of $S^{\circ}=S \backslash V$. A pleated surface (with pleating locus $T^{\prime}$ ) is a pair $(\tilde{\mathfrak{f}}, r)$, where $r: \pi_{1}\left(S^{\circ}\right) \rightarrow P S L(2, \mathbb{C})$ is a group homomorphism (not up to conjugacy), and $\widetilde{\mathfrak{f}}: \widetilde{S^{\circ}} \rightarrow \mathbb{H}^{3}$ is a map from the universal cover $\widetilde{S^{\circ}}$ of $S^{\circ}$ such that

- $\tilde{\mathfrak{f}}$ sends homeomorphically each component of the preimage $\widetilde{T}^{\prime}$ of $T^{\prime}$ in $\widetilde{S^{\circ}}$ to a complete geodesic in $\mathbb{H}^{3}$;

- $\tilde{\mathfrak{f}}$ sends homeomorphically the closure of each component of $\tilde{S}^{\circ} \backslash \widetilde{T}^{\prime}$ to an ideal triangle in $\mathbb{W}^{3}$;

- $\tilde{\mathfrak{f}}$ is $r$-equivariant, that is, for all $x \in \tilde{S^{\circ}}, \gamma \in \pi_{1}\left(S^{\circ}\right)$ we have $\tilde{\mathfrak{f}}(\gamma x)=r(\gamma) \tilde{\mathfrak{f}}(x)$.

Two pleated surfaces $(\tilde{\mathfrak{f}}, r)$ and $\left(\tilde{\mathfrak{f}}^{\prime}, r^{\prime}\right)$ are said isometric if there exists an isometry $A \in P S L(2, \mathbb{C})$ and a lift $\widetilde{\phi}: \widetilde{S^{\circ} \rightarrow} \widetilde{S}^{\circ}$ of an isotopy of $S^{\circ}$ such that $\tilde{\mathfrak{f}}^{\prime}=A \circ \tilde{f} \circ \tilde{\phi}$ and $r^{\prime}(\gamma)=A r(\gamma) A^{-1}$ for all $\gamma \in \pi_{1}\left(S^{\circ}\right)$. The set of isometry classes of pleated surfaces $(\tilde{\mathfrak{f}}, r)$ is in one-one correspondence with arrays $\left\{x_{e}\right\}_{e}$ of nonzero complex numbers $x_{e}$ associated to the edges $e$ of $T^{\prime}$, the exponential shear-bend parameters [8]. For instance, it is shown in [30] that the subset corresponding to real positive shear-bend parameters is real-analytic diffeomorphic to the Teichmüller space of hyperbolic metrics on $S^{\circ}$, with totally geodesic boundary completion or noncompact finite area completion at $v_{i}$ according to the type of the holonomy, loxodromic or parabolic. In fact, to any pleated surface $(\tilde{\mathfrak{f}}, r)$ we can associate a tuple $\mathcal{C}^{-}$of conjugacy class representatives of the boundary holonomies in the conjugacy class of $r$, as in Section 3.2.

In general, we can also associate a sign to each loxodromic puncture. Namely, if $A$ is a small annulus neighborhood of $v_{i}$, each connected component $\tilde{A}$ of the preimage 
of $A$ in $\widetilde{S^{\circ}}$ is the fixed point set of a subgroup $\pi$ of $\pi_{1}\left(S^{\circ}\right)$. This subgroup is the image of the fundamental group of $A$ for some choice of base points and paths between these base points. All the edges of $\widetilde{T}^{\prime}$ that meet $\tilde{A}$ are sent by $\tilde{f}$ to geodesics lines that meet at one of the two fixed points of $r(\pi)$. We specify this fixed point by a sign, as it determines an orientation (whence a generator) for the axis of the group $r(\pi)$. Since any two subgroups $\pi$ as above are conjugated, for each puncture the fixed point assignment is $r$-equivariant, so that the sign is canonically associated to the puncture.

Recall that in Definition 3.3 we used the boundary orientation to assign $\mathcal{C}_{i}^{-}$to the $i$-th puncture of $\partial F$. Let us remove this constraint, and associate to each boundary component with loxodromic type an orientation that we specify by a sign, positive for the boundary orientation, and negative otherwise. Then, for each tuple $\mathcal{C}^{-}$with $l$ loxodromic entries and each $l$-tuple of signs $s$, we get a space $\mathcal{Z}_{I}\left(T, b, \mathcal{C}^{-}, s\right)$. We define $W^{-}(T, b, \mathcal{C}, s)=W\left(\mathcal{Z}_{I}\left(T, b, \mathcal{C}^{-}, s\right)\right)$.

Proposition 3.11 For each tuple $\mathcal{C}^{-}$with nontrivial entries $\mathcal{C}_{i}^{-}, l$ being loxodromic, and for each $l$-tuple of signs $s$, the space $W^{-}(T, b, \mathcal{C}, s)$ coincides with the exponential shear-bend parameter space $\mathcal{P} \mathcal{S}_{\mathcal{C}}^{S}$ of isometry classes of pleated surfaces with boundary holonomies $\mathcal{C}^{-}$and signs $s$ :

$$
\mathcal{P} \mathcal{S}_{\mathcal{C}}^{S}=\left\{\left\{x_{e}\right\} \in(\mathbb{C} \backslash\{0\})^{-3 \chi\left(S^{\circ}\right)} \mid \text { for } i=1, \ldots, r, \prod x_{e}=\mu_{i}\right\} .
$$

Here the product is over all edges $e$ with $v_{i} \in \partial e$ (counted with multiplicities), $\mu_{i}$ is 1 if $\mathcal{C}_{i}^{-}$is parabolic, and, if $\mathcal{C}_{i}^{-}$is loxodromic, $\mu_{i}$ is the dilation factor of the generator of $\left\langle\mathcal{C}_{i}^{-}\right\rangle$specified by the sign of $v_{i}$. (Hence, in either case this is an eigenvalue of $\mathcal{C}_{i}^{-}$.)

Proof By the results of [8] recalled above, each point of $W^{-}(T, b, \mathcal{C}, s)$ is identified with the family of exponential shear-bend parameters of an isometry class of pleated hyperbolic surface on $S^{\circ}$ with pleated locus $T^{\prime}$. The definition of the map $\Theta: W^{-}(T, b, \mathcal{C}, s) \rightarrow \mathcal{R}_{I}\left(T, b, \mathcal{C}^{-}\right)$shows that this isometry class has $\mathcal{C}^{-}$as conjugacy class representatives of the boundary holonomies. Furthermore, by using the recipe given in the next paragraph for computing $\Theta$, we check that the holonomy of any positively oriented (with respect to the boundary orientation) small loop about the puncture $v_{i}$ is exactly $\mathcal{C}_{i}^{-}$. The upper left diagonal entry is just the product of a square root of the exponential $\mathcal{I}$-parameters at the edges with endpoint $v_{i}$. From (3-4), which is an easy consequence of results in [8, Sections 12.2-12.3], we deduce that $W^{-}(T, b, \mathcal{C}, s) \subset \mathcal{P} \mathcal{S}_{\mathcal{C}}^{s}$.

Conversely, for any $(\tilde{\mathfrak{f}}, r) \in \mathcal{P} \mathcal{S}_{\mathcal{C}}^{s}$ we have $\operatorname{conj}(r) \in \mathcal{R}_{I}\left(T, b, \mathcal{C}^{-}\right)$. It is also known that the isometry class of $(\tilde{\mathfrak{f}}, r)$ is determined by $r$ and the signs $s$ [9, Proposition 33]. As the map $\Theta$ is onto, let us take $z \in \mathcal{Z}_{I}\left(T, b, \mathcal{C}^{-}, s\right)$ with $\operatorname{conj} \circ f(z)=\operatorname{conj}(r)$ and 
consider the isometry class of pleated hyperbolic surfaces associated to $W^{-}(z)$. There is a representative $\left(\tilde{\mathfrak{f}}^{\prime}, r\right)$ with the same holonomy $r$. As the signs of $\left(\tilde{\mathfrak{f}}^{\prime}, r\right)$ are $s$, the same as for $(\tilde{\mathfrak{f}}, r)$, the two pleated surfaces coincide. So $\mathcal{P} \mathcal{S}_{\mathcal{C}}^{s} \subset W^{-}(T, b, \mathcal{C}, s)$.

Remark 3.12 Exponential shear-bend parameters do not depend on branchings but the orientation of $F$. (In fact, by taking the edge orientation opposite to the branching one in the definition of $W^{-}(z)$, the left and right triangles are exchanged so that the result is the same). However, the interpretation as $(-)$-exponential $\mathcal{I}$-parameters is meaningful for QHFT because branchings govern all the choices in QHFT tensors, and these parameters are defined simultaneously as the $(+)$-ones from 1-cocycles on the triangulated boundary of arbitrary compact orientable 3-manifolds (eg not necessarily mapping cylinders). When $\mathcal{C}$ has no trivial entries, the maps $W^{ \pm}$define decorated shear-bend parameter spaces similar to those occurring in $[21 ; 26]$.

Remark 3.13 For tuples $\mathcal{C}$ with no trivial entries we can use simpler $e$-triangulations of $F$ to define exponential $\mathcal{I}$-parameters (see Figure 8). Namely, for each of the triangles $t_{v}$ of the base surface $S$ we remove the interior of a monogon inside $t_{v}$, and triangulate the resulting quadrilateral by adding an edge $e_{v}$ with endpoints $v$ and the $b$-output vertex of the opposite edge. We extend $b$ by orienting $e_{v}$ from that vertex to $v$. Proposition 3.4 applies to the cocycle parameters based on such $e$-triangulations, and the treatment of exponential $\mathcal{I}$-parameters works as well.

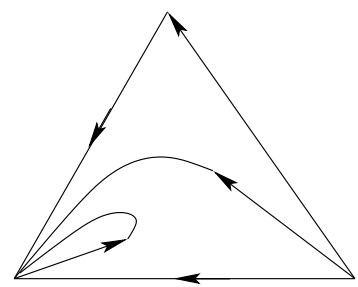

Figure 8: More economic e-triangulation

Geometry and computation of $\Theta^{ \pm}$The maps $\Theta^{ \pm}$in (3-3) can be defined directly in terms of $\mathcal{I}$-parameters. A way to see this is to consider $\mathcal{I}$ triangulations of cylinders $C=F \times[0,1]$, as discussed in Section 5.5, and the corresponding pseudodeveloping maps. We give here another description. Let us just consider $\Theta^{-}$. Recall that $\operatorname{PSL}(2, \mathbb{C})$ is isomorphic to Isom ${ }^{+}\left(\mathbb{M}^{3}\right)$, with the natural conformal action on $\mathbb{C P} \mathbb{P}^{1}=\partial \overline{\mathbb{T}}^{3}$ via linear fractional transformations. For any $\rho \in \mathcal{R}\left(g, r, \mathcal{C}^{-}\right)$, take a representative $\tilde{\rho}: \pi_{1}(F, q) \rightarrow P S L(2, \mathbb{C})$ in the conjugacy class. Consider the 
associated flat principal $\operatorname{PSL}(2, \mathbb{C})$-bundle $F_{\widetilde{\rho}}$. A trivializing atlas defines a cocycle $z \in \mathcal{Z}\left(T, b, \mathcal{C}^{-}\right)$. If $\rho \in \mathcal{R}_{I}\left(T, b, \mathcal{C}^{-}\right)$, we can take $z \in \mathcal{Z}_{I}\left(T, b, \mathcal{C}^{-}\right)$, so that the trivializing atlas of $F_{\widetilde{\rho}}$ associated to the cellulation $T^{*}$ dual to $T^{\prime}$, with edges oriented by using the orientation of $F$ and the branching orientation of the edges of $T^{\prime}$, has nontrivial transition functions. These can be viewed as transition functions for the fiber bundle associated to $F_{\tilde{\rho}}$ and with fibre $\mathbb{H}^{3}$. For each $2-\operatorname{simplex} t$ of $T^{\prime}$, there is a unique $g_{t} \in \operatorname{Isom}\left(\mathbb{H}^{3}\right)$ (possibly reversing the orientation) mapping the vertices $u_{0}$, $u_{1}$ and $u_{2}$ of the idealization of $t$ to $0, \infty$ and -1 respectively. Then, the transition function along the edge of $T^{* *}$ positively transverse to a given edge $e$ of $T^{\prime}$ is of the form $\left(g_{t_{l}}\right)^{-1} \circ \varphi(z)(e) \circ g_{t_{l}} \in \operatorname{Isom}^{+}\left(\mathbb{M}^{3}\right)$, where $t_{l}$ is the triangle on the left of $e$, and $\varphi(z)(e)$ is the isometry of $\mathbb{H}^{3}$ of hyperbolic type fixing 0 and $\infty$ and mapping 1 to $W^{-}(z)(e)$. Analytic continuation defines the parallel transport of $F_{\widetilde{\rho}}$ along paths transverse to $T^{\prime}$, whence a representation into $\operatorname{PSL}(2, \mathbb{C})$ of the groupoid of such paths, well-defined up to homotopy $\operatorname{rel}(\partial)$. In particular, it gives a practical recipe to compute $\tilde{\rho}$, that we describe now.
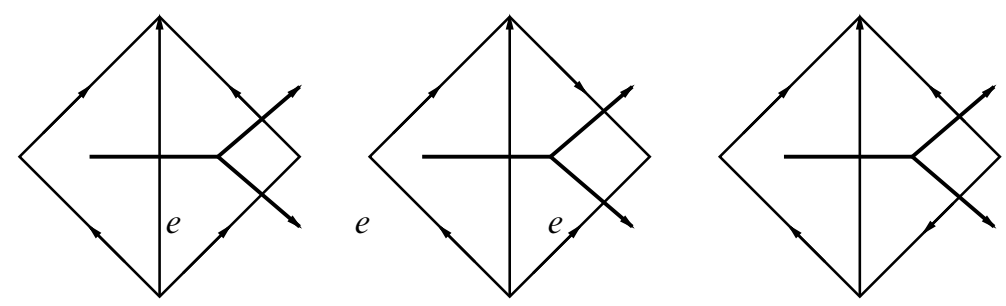

Figure 9: The recipe for reading off holonomies from exponential $\mathcal{I}$-parameters

Let the base point $q$ be not in the 1-skeleton of $T^{\prime}$. Given an element of $\pi_{1}(F, q)$, represent it by a closed curve $\gamma$ in $F$ transverse to $T^{\prime}$, and which does not depart from an edge it just entered. Assume that $\gamma$ intersects an edge $e$ of $T^{\prime}$ positively with respect to the orientation of $F$. Figure 9 shows three possible branching configurations for the two triangles glued along $e$. Fix arbitrarily a square root $W^{-}(z)(e)^{1 / 2}$ of $W^{-}(z)(e)$. Consider the elements of $\operatorname{PSL}(2, \mathbb{C})$ given by

$$
\gamma(e)=\left(\begin{array}{cc}
W^{-}(z)(e)^{1 / 2} & 0 \\
0 & W^{-}(z)(e)^{-1 / 2}
\end{array}\right), \quad p=\left(\begin{array}{ll}
0 & 1 \\
1 & 0
\end{array}\right), \quad l=\left(\begin{array}{ll}
-1 & 1 \\
-1 & 0
\end{array}\right)
$$

and $r=l^{-1}$. The matrix $\gamma(e)$ represents the isometry with fixed points $0, \infty \in \mathbb{C P}^{1}$ and mapping 1 to $W^{-}(z)(e)$. The elliptic elements $p$ and $l$ send $(0,1, \infty)$ to $(\infty, 1,0)$ 
and $(\infty, 0,1)$ respectively. For the portion of $\gamma$ on the left of Figure 9 , if $\gamma$ turns to the left after crossing $e$ the parallel transport operator is $\gamma(e) \cdot p \cdot l$, while it is $\gamma(e) \cdot p \cdot r$ if $\gamma$ turns to the right. (The matrix multiplication is on the right, as is the action of $\operatorname{PSL}(2, \mathbb{C})$ on the total space of $\left.F_{\tilde{\rho}}\right)$. In the middle and right pictures the parallel transport operators along the portion of $\gamma$ are given by $\gamma(e) \cdot l$ or $\gamma(e) \cdot p \cdot l$, and $\gamma(e) \cdot p \cdot r$ or $\gamma(e) \cdot r$ respectively. We see that the action of $p, l$ and $r$ depends on the reordering of the vertices after the mapping $\gamma(e)$. If $\gamma$ intersects $e$ negatively, we replace $\gamma(e)$ with $\gamma(e)^{-1}$ in the above expressions. A similar recipe applies for any other branching of the two triangles glued along $e$.

Continuing this way each time $\gamma$ crosses an edge of $T^{\prime}$ until it comes back to $q$, we get an element $[W(z)]_{q}(\gamma) \in \operatorname{PSL}(2, \mathbb{C})$ that depends only on the homotopy class of $\gamma$ based at $q$ and coincides with $\tilde{\rho}(\gamma)$, because of the identity $D_{z}(\gamma \cdot x)=$ $[W(z)]_{q}(\gamma) \cdot D_{z}(x)$ for any $x \in \tilde{F}$, where $D_{z}: \widetilde{F} \rightarrow \mathbb{C P}^{1}$ is a $\tilde{\rho}$-equivariant pseudodeveloping map from the universal cover $\widetilde{F}$ of $F$. Varying $\gamma$, we eventually get the representation $[W(z)]_{q}=\tilde{\rho}: \pi_{1}(F, q) \rightarrow \operatorname{PSL}(2, \mathbb{C})$.

\section{The QHFT bordism category}

We define first a topological $(2+1)$-bordism category. Then we will give it more structure, including the parameter spaces of the previous sections.

\subsection{Marked topological bordisms}

Like in Section 3, for every $(g, r) \in \mathbb{N} \times \mathbb{N}$ such that $g \geq 0, r>0$, and $r>2$ if $g=0$, fix a compact closed oriented base surface $S$ of genus $g$ with a set $V=\left\{v_{1}, \ldots, v_{r}\right\}$ of $r$ marked points. Denote by $-S$ the same surface with the opposite orientation, and write $* S$ for $S=+S$ or $-S$. Moreover, fix a set of disjoint embedded closed segments $a_{v_{i}}$ in $S$ such that $a_{v_{i}}$ has one end point at $v_{i}$. We say that $v_{i}$ is framed by $a_{v_{i}}$.

We say that two orientation preserving diffeomorphisms $\phi_{1}: * S \rightarrow \Sigma_{1}$ and $\phi_{2}: * S \rightarrow$ $\Sigma_{2}$ are equivalent if there is an orientation-preserving diffeomorphism $h: \Sigma_{1} \rightarrow \Sigma_{2}$ such that $\left(\phi_{2}\right)^{-1} \circ h \circ \phi_{1}$ pointwise fixes the segments $a_{v_{i}}$ and is isotopic to the identity automorphism of $S$ relatively to $\left\{a_{v_{i}}\right\}$. We write $[* S, \phi]$ for such an equivalence class.

Let $Y$ be an oriented compact 3-manifold with (possibly empty) boundary $\partial Y$, with an input/output bipartition $\partial Y=\partial_{-} Y \cup \partial_{+} Y$ of the boundary components (we say that $\partial_{-} Y$ is "at the bottom" of $Y$, while $\partial_{+} Y$ is "on the top"). Each boundary component inherits the boundary orientation, via the convention last is the ingoing normal. 
Let $L_{\mathcal{F}}$ be a nonempty framed tangle in $Y$. This means that

$$
L_{\mathcal{F}}=\left(L_{\mathcal{F}}\right)_{b} \coprod\left(L_{\mathcal{F}}\right)_{i}
$$

is a disjoint union of ribbons, that is, properly embedded quadrilaterals and orientable annuli: $\left(L_{\mathcal{F}}\right)_{i} \subset \operatorname{Int}(Y)$ is made of the components homeomorphic to $S^{1} \times[0,1]$, while $\left(L_{\mathcal{F}}\right)_{b}$ is made of the components homeomorphic to $I \times[0,1]$, which intersect $\partial Y$ along the image of $\partial I \times[0,1]$ in $Y$. Associated to $L_{\mathcal{F}}$ we have an unframed tangle $L$, obtained by collapsing each component to its core, homeomorphic to $S^{1}$ or $I$.

We consider $L_{\mathcal{F}}$ up to proper ambient isotopy. For every boundary component $\Sigma$ of $Y$, we assume that $\left(L_{\mathcal{F}}\right)_{b} \cap \Sigma \neq \varnothing$, and that $\left(L_{\mathcal{F}}\right)_{b} \cap \Sigma$ consists of at least two segments if $g(\Sigma)=0$ (we do not require that every component of $\left(L_{\mathcal{F}}\right)_{b}$ goes from $\partial_{-}$ towards $\left.\partial_{+}\right)$. Hence, on each boundary component $\Sigma$ of $Y$ we have a set of marked points framed by $L_{\mathcal{F}} \cap \Sigma$. Also, associated to $\partial_{ \pm}$we have a finite disjoint union $\alpha_{ \pm}=\bigsqcup_{\phi(* S) \in \partial_{ \pm}}[* S, \phi]$ of equivalence classes of diffeomorphisms as above.

Consider the topological $(2+1)$-bordism category with objects the empty set and any finite union of the $[* S, \phi]$, and morphisms the triples $\left(Y, L_{\mathcal{F}}, \alpha_{ \pm}\right)$as above. We say that $\left(Y, L_{\mathcal{F}}, \alpha_{ \pm}\right)$is a bordism from $\alpha_{-}$to $\alpha_{+}$with support $\left(Y, L_{\mathcal{F}}\right)$. We allow the case when $Y$ is a closed manifold, so that $\partial Y=\varnothing$ and $\left(Y, L_{\mathcal{F}}\right)$ is a morphism from the empty set to itself. We stress that $L_{\mathcal{F}}$ is nonempty in any case.

We can reformulate this category in a setup closer to that of the phase space parameters of Section 3, as follows. If we cut open each $a_{v_{i}}$ in $S$ we get an oriented surface $F$ with $r$ boundary bigon components. This is the domain of an elementary object $[* F, \phi]$, where the target surfaces $\Sigma$ have now $r$ boundary components, and the diffeomorphisms $\phi$ are considered up to isotopy $\operatorname{rel}(\partial)$.

Consider a bordism $\left(Y, L_{\mathcal{F}}, \alpha_{ \pm}\right)$. On the boundary of each component of $L_{\mathcal{F}}$ we have the tangle line and the longitudinal line, corresponding respectively to $X \times\{0\}$ and $X \times\{1\}$ in the annulus or quadrilateral $X \times[0,1]\left(X=S^{1}\right.$ or $\left.I\right)$. The longitudinal line specifies a framing of the normal bundle of the parallel tangle line. The tangle and longitudinal lines of all components of $L_{\mathcal{F}}$ determine a pair $\bar{\lambda}=\left(\lambda, \lambda^{\prime}\right)$ of unframed tangles in $Y$. Cutting open each ribbon we get a 3 -manifold with corners $\widetilde{Y}$. The boundary $\partial \tilde{Y}$ has two "horizontal" parts $\partial_{ \pm} \tilde{Y}$ contained in $\partial_{ \pm} Y$, and a "vertical" tunnel part $\widetilde{L}_{\mathcal{F}}$. The horizontal parts intersect the tunnel part at the corner locus; this is a union of bigons contained in $\partial Y$. Each boundary component $\Sigma$ of $Y$ corresponds to a horizontal boundary component of $\tilde{Y}$, still denoted by $\Sigma$. Each tunnel boundary component is made by the union of two copies of $X \times(0,1)$, glued each to the other at $\lambda \cup \lambda^{\prime}$. The horizontal boundary components are the targets of elementary objects $[* F, \phi]$, and each triple $\left(\tilde{Y}, \tilde{L}_{\mathcal{F}}\right)$ supports a morphism between such objects. Clearly, 
we can recover $\left(Y, L_{\mathcal{F}}\right)$ from $\left(\tilde{Y}, \widetilde{L}_{\mathcal{F}}\right)$, so that we have two equivalent settings to describe the same topological bordism category.

\subsection{Boundary structures}

We equip a marked topological bordism with additional boundary structures by using the notions of Section 3. Fix an $e$-triangulation $(T, b)$ of $F$. Following Remark 2.4 and the definition of log-branches, we put:

Definition 4.1 Let $z \in \mathcal{Z}_{I}(T, b)^{ \pm}$, and $e$ be a nonboundary edge of $T$. Denote $1_{z}(e)$ the canonical log-branch of $W^{ \pm}(z)(e)$, computed from the idealization of $\operatorname{Star}(e)$ as in Lemma 2.13 and before Definition 3.9. For any collection $m=\left\{m_{\lambda}\right\}_{\lambda}$ of integers, one for each edge $\lambda$ in $T$, the classical $\log -\mathcal{I}$-parameter of $(z, m)$ at $e$ is

$$
1_{(z, m)}(e)=1_{z}(e)+\sqrt{-1} \pi\left(m_{a}+m_{c}-m_{b}-m_{d}\right),
$$

where $a, \ldots, d$ make $\operatorname{Link}(e)$ with $a, c$ opposite and $e, a$ have coherent branching orientations (see Figure 10). Similarly, for every $N>1$ and any other collection $n=\left\{n_{\lambda}\right\}_{\lambda}$ of integers, the quantum $\log -\mathcal{I}$-parameter at $e$ is

$\mathrm{l}_{(z, m)}(e)+\sqrt{-1} \pi N\left(m_{a}+m_{c}-m_{b}-m_{d}\right)-*_{b} \sqrt{-1} \pi(N+1)\left(n_{a}+n_{c}-n_{b}-n_{d}\right)$.

We call the collections

and $c=\left\{c_{e}\right\}_{e}=\left\{n_{a}+n_{c}-n_{b}-n_{d}\right\}_{e}$

$$
f=\left\{f_{e}\right\}_{e}=\left\{\left(1_{(z, m)}(e)-\log \left(W^{ \pm}(z)(e)\right)\right) / \pi \sqrt{-1}\right\}_{e}
$$

the flattenings of $(z, m)$ and the charge of $n$ respectively, and we denote generically by $\mathcal{L}$ any such a system of classical or quantum $( \pm)-\log -\mathcal{I}$-parameters.

Remark that if $z \in \mathcal{Z}_{I}\left(T, b, \mathcal{C}^{-}\right)$and $\mathcal{C}$ has no trivial entries, then the (-)-Log- $\mathcal{I}_{-}$ parameters depend on $W^{-}(z)$, not on $z$, because of Proposition 3.10.

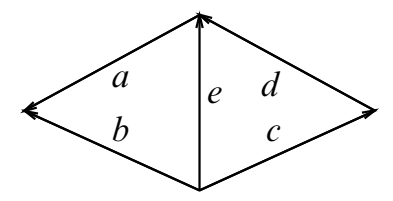

Figure 10: Notation for $\log -\mathcal{I}$-parameters 
Definition 4.2 The QHFT category is the $(2+1)$-bordism category with objects the empty set and any finite union of the $[* F,(T, b, \mathcal{L}), \phi]$, where $\mathcal{L}$ is a system of $(+)-\log -\mathcal{I}$-parameters. The morphisms are the 4 -tuples $\left(\widetilde{Y}, \widetilde{L}_{\mathcal{F}}, \rho, \alpha_{ \pm}\right)$, where $\rho$ is a conjugacy class of $\operatorname{PSL}(2, \mathbb{C})$-valued representations of $\pi_{1}\left(\widetilde{Y} \backslash \widetilde{L}_{\mathcal{F}}\right)$, and $\alpha_{ \pm}$are QHFT objects with targets $\partial_{ \pm} \tilde{Y}$, such that for every $[* F,(T, b, \mathcal{L}), \phi]$, the character $\phi^{*}(\rho)$ coincides with $\Theta\left(W^{+}(z)\right)$. We say that $\left(\tilde{Y}, \widetilde{L}_{\mathcal{F}}, \rho\right)$ is the support of a QHFT bordism from $\alpha_{-}$to $\alpha_{+}$, and that $\alpha_{ \pm}$is a QHFT surface.

Given bordisms $\mathcal{B}$ and $\mathcal{B}^{\prime}$ from $\alpha_{-}$to $\alpha_{+}$and $\alpha_{-}^{\prime}$ to $\alpha_{+}^{\prime}$, respectively, assume that $\beta_{+}$and $\beta_{-}^{\prime}$ are subobjects of $\alpha_{+}$and $\alpha_{-}^{\prime}$ that coincide up to the change of orientation.

Definition 4.3 The bordism $\mathcal{B}^{\prime \prime}$ from $\alpha_{-}^{\prime \prime}=\alpha_{-} \cup\left(\alpha_{-}^{\prime} \backslash \beta_{-}^{\prime}\right)$ to $\alpha_{+}^{\prime \prime}=\alpha_{+}^{\prime} \cup\left(\alpha_{+} \backslash \beta_{+}\right)$ obtained by gluing $\mathcal{B}$ and $\mathcal{B}^{\prime}$ along $\beta_{+}$is called the composition of $\mathcal{B}$ followed by $\mathcal{B}^{\prime}$. We write $\mathcal{B}^{\prime \prime}=\mathcal{B}^{\prime} * \mathcal{B}$.

Examples from hyperbolic geometry Any topologically tame hyperbolic 3-manifold $Y$ with hyperbolic holonomy $\rho$ and a tangle $L_{\mathcal{F}}$ of singularities makes a QHFT bordism. More specifically, any geometrically finite noncompact complete hyperbolic 3-manifold $Y$ defines a triple $\left(Y^{\prime}, L_{\mathcal{F}}, \rho\right)$ with a nonempty link $L_{\mathcal{F}}$, as follows. The manifold $Y$ has a natural compactification $\bar{Y}$, with $Y \cong \operatorname{Int}(\bar{Y})$, which is a "pared" manifold $(\bar{Y}, P)$. Here $P$ is a union of disjoint tori or annuli embedded in the boundary of $\bar{Y}$. The tori correspond to the cusps of $Y$. Each annulus $A$ of $P$ comes from a couple of cusps on some boundary component of (a small neighborhood of) the convex core of $Y ; A$ is fibered by geodesic arcs. If $A$ is separating the cusps belong to different components. Define $Y^{\prime \prime}$ as the result of attaching a 2-handle to $\bar{Y}$ at each annulus $A$, so that $P$ is contained in the interior of $Y^{\prime \prime}$ and is transverse to $\partial Y^{\prime \prime}$. Equivalently, $Y^{\prime \prime}$ contains a properly embedded framed 1-tangle $L_{\mathcal{F}}^{\prime}$ made by the cocores of the 2-handles, the framing being determined by the fibration by intervals of the annuli of $P$. Let us choose a framing at each torus of $P$. By Dehn filling we get a manifold $Y^{\prime}$, and $Y^{\prime \prime}$ is the exterior in $Y^{\prime}$ of the union $L_{\mathcal{F}}^{\prime \prime}$ of the framed cores of the filling solid tori. Hence, if every boundary component of the convex core of $Y$ contains at least one cusp, associated to $Y$ and the cusp framings we have $\left(Y^{\prime}, L_{\mathcal{F}}, \rho\right)$, where $L_{\mathcal{F}}=L_{\mathcal{F}}^{\prime} \cup L_{\mathcal{F}}^{\prime \prime}$ intersects all the boundary components of $Y^{\prime}$, and $\rho$ is a $P S L(2, \mathbb{C})$-character of $Y^{\prime} \backslash L_{\mathcal{F}} \cong Y$. If furthermore $\rho$ is the holonomy of a complete hyperbolic metric on $Y$ and $Y$ has infinite volume ends, then $\partial Y^{\prime}$ is nonempty. We can give the triple $\left(Y^{\prime}, L_{\mathcal{F}}, \rho\right)$ a natural boundary structure $\alpha_{-} \cup \alpha_{+}$, induced by exponential $\mathcal{I}$-parameters of the pleated surfaces in the boundary of the convex core (see eg Epstein and Marden [14]). 


\section{The QHFT functor}

Consider a QHFT bordism $\mathcal{B}=\left(\tilde{Y}, \widetilde{L}_{\mathcal{F}}, \rho, \alpha_{ \pm}\right)$. For every odd integer $N \geq 1$, we associate a finite dimensional complex linear space $V\left(\alpha_{ \pm}\right)$to $\alpha_{ \pm}$, and a linear map $\mathcal{H}_{N}(\mathcal{B}): V\left(\alpha_{-}\right) \rightarrow V\left(\alpha_{+}\right)$to $\mathcal{B}$, well-defined up to sign and multiplication by $N$-th roots of unity. This defines a (moderately projective) functor $\mathcal{H}_{N}$ : QHB $\rightarrow$ Vect, where Vect is the tensor category of complex linear spaces. The construction immediately implies that $\mathcal{H}_{N}$ is a modular functor, in the sense of [32, III.1.2].

\subsection{From QHFT bordisms to QHG-triangulated pseudomanifolds}

First we associate to $\mathcal{B}$ a pseudomanifold $Z(\mathcal{B})$. Fill each tunnel boundary component of $\tilde{Y}$ with a solid tube, thus recovering a copy of the manifold $Y$. The cores of the solid tubes make a parallel unframed copy $\lambda^{\prime \prime}$ of $L$. We define $Z(\mathcal{B})$ as the result of collapsing to one point each component of $\lambda^{\prime \prime}$. In other words, we glue to each tunnel component of $\tilde{Y}$ the oriented topological cusp $\widehat{\mathbf{C}}=B \times[0,+\infty] /(B \times\{\infty\})$ with base equal to either $B=S^{1} \times[-1,1]$ or $B=S^{1} \times S^{1}$.

Next we describe a procedure to convert $Z(\mathcal{B})$ to a distinguished QHG-triangulated pseudomanifold. We refer to the notions introduced in Section 2.4.

We say that a branched triangulation $(T, b)$ of $B$ as above is admissible if $B \cap \partial_{ \pm} \tilde{Y}$ and the tangles $\lambda, \lambda^{\prime}$ are covered by the $1-$ skeleton. We denote by $(\widehat{T}, \widehat{b})$ the branched triangulation of $\widehat{\mathbf{C}}$, where $\widehat{T}$ is the cone over $T$ from the nonmanifold point, say $\infty$, and $\hat{b}$ extends $b$ so that $\infty$ is a pit for every branched tetrahedron of $\widehat{T}$. Assume we are given an idealizable $P B^{+}(2, \mathbb{C})$-valued cocycle $z$ on an admissible triangulation of $B$. The idealization of $z$ determines for each 2-simplex of $B$ a face of an ideal hyperbolic tetrahedron with further vertex at $\infty$ (see Figure 11, where opposite vertical triangles are identified). Since the fundamental group of $B$ is abelian, the resulting family of oriented ideal hyperbolic tetrahedra actually makes an $\mathcal{I}$-triangulation $(\widehat{T}, \hat{b}, w)$, which we call an $\mathcal{I}$-cusp. By conjugating if necessary, we see that the notion of $\mathcal{I}$-cusp makes sense also when $z$ takes values more generally in $\operatorname{PSL}(2, \mathbb{C})$. We get flattenings similarly as in Lemma 2.13: at a corner of a 2-simplex formed by edges $e_{l}$ and $e_{r}$ we put the difference of the logarithms of the vectors in $\mathbb{C}$ associated by the idealization to $e_{l}$ and $e_{r}$, respectively.

Definition 5.1 A $\mathcal{D}$-triangulation of $\mathcal{B}=\left(\tilde{Y}, \widetilde{L}_{\mathcal{F}}, \rho, \alpha_{ \pm}\right)$consists of a 4 -tuple $\mathcal{K}=(K, \bar{H}, b, z)$ where:

(a) $(K, b)$ is a branched triangulation of $\tilde{Y}$ extending that on $\partial_{ \pm} \tilde{Y}$, and inducing an admissible cusp base triangulation at each tunnel component of $\widetilde{L}_{\mathcal{F}}$. 


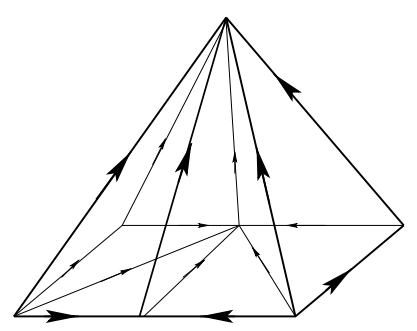

Figure 11: An $\mathcal{I}$-cusp

(b) The 1-dimensional subcomplex $\bar{H}=H \cup H^{\prime}$ of $K$ is ambiently isotopic to the tangle $\bar{\lambda}=\lambda \cup \lambda^{\prime}$, and $\bar{H}$ contains all the vertices of $K$.

(c) $z$ is an idealizable $\operatorname{PSL}(2, \mathbb{C})$-valued 1 -cocycle on $(K, b)$ such that:

(i) the conjugacy class of $\operatorname{PSL}(2, \mathbb{C})$-representations of $\pi_{1}(\tilde{Y})$ associated to $z$ coincides with $\rho$;

(ii) the (+)-exponential $\mathcal{I}$-parameters given by the restriction of $z$ to $\partial_{ \pm} \tilde{Y}$ coincide with that of the objects $\alpha_{-} \cup \alpha_{+}$(see Definition 4.1).

(d) the restriction of $z$ to each vertical tunnel component of $\tilde{L}_{\mathcal{F}}$ takes values in the Borel subgroup $P B^{+}(2, \mathbb{C})$ of $P S L(2, \mathbb{C})$.

For any $\mathcal{D}$-triangulation $\mathcal{K}=(K, \bar{H}, b, z)$ of $\mathcal{B}$ we get a distinguished $\mathcal{I}$-triangulation $\mathcal{K}_{\mathcal{I}}=(K, \bar{H}, b, w)$ of $(Z(\mathcal{B}), \bar{\lambda})$ by gluing the idealization of $\mathcal{K}$ with the $\mathcal{I}$-cusp given by the cocycle at each tunnel component. Note that $\bar{H}$ contains all the vertices of $K_{I}$ that are manifold points.

Definition 5.2 We say that $\mathcal{T}(\mathcal{B})=\left(\mathcal{K}_{\mathcal{I}}, f, c\right)=(K, \bar{H}, b, w, f, c)$ is a distinguished flat/charged $\mathcal{I}$-triangulation of $(Z(\mathcal{B}), \bar{\lambda})$ if it satisfies Definition 2.17 , and at every boundary edge of $Z(\mathcal{B})$ the total (classical or quantum) log-branch of $\left(\mathcal{K}_{\mathcal{I}}, f, c\right)$ of $(2-18)$ coincides with the (classical or quantum) $\log -\mathcal{I}$-parameter of the boundary object $\alpha_{-} \cup \alpha_{+}$.

Recall the cohomological weights from Section 2.4. These notions still make sense for distinguished flat/charged $\mathcal{I}$-triangulations, where the homology of $\partial T_{0}$ is replaced with that of the tunnel components $\widetilde{L}_{\mathcal{F}}$. Since we have weights $\left(h_{f}, k_{f}\right)$ for logbranches and $\left(h_{c}, k_{c}\right)$ for charges at the same time, we will denote them $(h, k)=$ $\left(\left(h_{f}, h_{c}\right),\left(k_{f}, k_{c}\right)\right)$. We can also define, in the very same way, boundary weights $k_{f} \in H^{1}(\partial \tilde{Y} ; \mathbb{C})$, but these are completely encoded by $\alpha_{ \pm}$. We have: 
Theorem 5.3 For every $\mathcal{B}=\left(\tilde{Y}, \widetilde{L}_{\mathcal{F}}, \rho, \alpha_{ \pm}\right)$and every $(h, k) \in H^{1}(\tilde{Y} ; \mathbb{Z} / 2 \mathbb{Z})^{2} \times$ $H^{1}\left(\widetilde{L}_{\mathcal{F}} ; \mathbb{C}\right)^{2}$ such that $\left(h_{f}, k_{f}\right)$ satisfies $(2-26)$, there are distinguished flat/charged $\mathcal{I}$-triangulations $\mathcal{T}(\mathcal{B})$ of $Z(\mathcal{B})$ with weight $(h, k)$, and any two are $Q H G$-isomorphic.

Proof The existence of distinguished $\mathcal{I}$-triangulations $\mathcal{K}_{\mathcal{I}}$ of $Z(\mathcal{B})$ follows from a tedious but straightforward generalization of Theorem 4.13 in [3]. Global flattenings and integral charges with arbitrary weight exist on the double $D \mathcal{K}_{\mathcal{I}}$ of $\mathcal{K}_{\mathcal{I}}$ by the results recalled with Theorem 2.20.

Consider the $(+)-\log -\mathcal{I}$-parameters $\left\{W^{ \pm}(z)(e)\right\}_{e}$ at $\alpha_{ \pm}$. They are in one-one correspondence with the interior edges of the corresponding $e$-triangulations, which is less than the cardinality of the families $m$ and $n$ used to define flattenings and charges in Definition 4.1. Hence any family of determinations of the logarithms of the $W^{ \pm}(z)(e)$ is a system of $(+)-\log -\mathcal{I}$-parameters. Also, Lemma 2.13 implies that any system of $(+)-\log -\mathcal{I}$-parameters at $\alpha_{ \pm}$extends to a distinguished flat/charged $\mathcal{I}$-triangulation of the pseudomanifold obtained from the trivial cylinders over $\partial_{ \pm} \tilde{Y}$ by collapsing to a point each annulus of $\partial\left(\partial_{ \pm} \tilde{Y}\right) \times[-1,1]$.

This means that any QHFT surface bounds a QHFT bordism, and that for the bordism $\mathcal{B}$ there are flat/charges on $D \mathcal{K}_{\mathcal{I}}$ whose restriction to $\mathcal{K}_{\mathcal{I}}$ induce the $\log -\mathcal{I}$-parameters of $\alpha_{ \pm}$. Hence we get global flat/charges as in Definition 5.2. In fact the affine spaces of flat/charges on $D \mathcal{K}_{\mathcal{I}}$ project onto that on $\mathcal{K}_{\mathcal{I}}$ compatible with $\alpha_{ \pm}$(see the end of Section 2). Then, the Mayer-Vietoris exact sequence in cohomology for the triad $\left(D K_{\mathcal{I}}, \mathcal{K}_{\mathcal{I}},-\mathcal{K}_{\mathcal{I}}\right)$ shows that $(h, k)$ is induced by some weight on $D \mathcal{K}_{\mathcal{I}}$. As we can choose the latter arbitrarily, this concludes the proof of the first claim.

The second is harder, but follows strictly from the arguments in the proof of Theorem 6.8 (2) in [4]. The only new ingredient is the presence of $\mathcal{I}$-cusps, which mimic the ends of cusped manifolds treated in that paper.

An alternative characterization of classical/quantum log-branches of distinguished flat/charged $\mathcal{I}$-triangulations $\mathcal{T}(\mathcal{B})$ follows from Remark 2.18 .

\subsection{Amplitudes}

Fix an odd positive integer $N$. Write $V=\mathbb{C}^{N}$, with the canonical basis $\left\{e_{i}\right\}$, and $V^{-1}$ for the dual space. Both are endowed with the hermitian inner product with orthonormal basis the vectors $e_{0}$ and $\left(e_{i}+e_{N-i}\right) / \sqrt{2}, i=1, \ldots, N-1$.

Recall the notation of Section 3. For each base surface $F$ fix an $e$-triangulation $(T, b)$, an ordering of the set $T^{(2)}$ of 2-simplices, and let $V(T, b)=\otimes_{t \in T^{(2)}} V^{\sigma_{ \pm}(t)}$. Given a 
QHFT bordism $\mathcal{B}=\left(\tilde{Y}, \tilde{L}_{\mathcal{F}}, \rho, \alpha_{ \pm}\right)$with a distinguished flat/charged $\mathcal{I}$-triangulation $\mathcal{T}(\mathcal{B})$, the trace tensor in $(2-22)$ is a morphism $\mathcal{H}_{N}(\mathcal{T}(\mathcal{B})) \in \operatorname{Hom}\left(V\left(\alpha_{-}\right), V\left(\alpha_{+}\right)\right)$, where $V\left(\alpha_{ \pm}\right)$is the tensor product of isomorphic copies of the spaces $V(T, b)$ over the (ordered) components of $\alpha_{ \pm}$.

Theorem 5.4 The morphism $\mathcal{H}_{N}(\mathcal{B}, h, k)=\mathcal{H}_{N}(\mathcal{T}(\mathcal{B}))$ does not depend on the choice of $\mathcal{T}(\mathcal{B})$ up to sign and multiplication by $N$-th roots of unity. We also have $\mathcal{H}_{N}(\mathcal{B}, h, k)=\mathcal{H}_{N}\left(\mathcal{B}, h^{\prime}, k^{\prime}\right)$ if the reductions $\bmod (N \mathbb{Z})$ of $h$ and $h^{\prime}$ (resp. $k$ and $\left.k^{\prime}\right)$ are the same, that is, if $k-k^{\prime} \in H^{1}\left(\widetilde{L}_{\mathcal{F}} ; \mathbb{Z}\right)$ and $k-k^{\prime}=0 \in H^{1}\left(\tilde{L}_{\mathcal{F}} ; \mathbb{Z} / N \mathbb{Z}\right)$, and similarly for $h$ and $h^{\prime}$. Moreover, by restricting to even-valued flattenings as in Remark 2.14 there is no sign ambiguity and $h=0$. We call $\mathcal{H}_{N}(\mathcal{B}, h, k)$ the amplitude of $(\mathcal{B}, h, k)$.

Proof The result is an immediate consequence of Proposition 2.7-Proposition 2.16 and the last claim in Theorem 5.3. For even-valued flattenings we have $h=0$, because any curve normal to the triangulation selects an even number of edges which are of the type $e_{2}$ or the opposite in the (branched) tetrahedra traversed by the path (the manifold is orientable, and the branching orientations of the faces adjacent to such edges are reversed when we follow the curve). For the dependance with respect to the $\bmod (N \mathbb{Z})$ reductions of weights, we note that the associated systems of $N$-th roots of moduli (see Remark 2.18) are connected by QHG isomorphisms. Indeed, the difference $k-k^{\prime} \in H^{1}\left(\tilde{L}_{\mathcal{F}} ; N \mathbb{Z}\right)$ coincides with $\gamma(f)-\gamma\left(f^{\prime}\right) / \sqrt{-1} \pi$ and $\gamma(c)-\gamma\left(c^{\prime}\right) / \sqrt{-1} \pi$ for some $f, f^{\prime}$ and $c, c^{\prime}$, and similarly for $h-h^{\prime}$ and the $\gamma_{2}$ maps. By first considering $\left(k-k^{\prime}\right) / N$ we can eventually take the collections of values of $f-f^{\prime}$ and $c-c^{\prime}$ in $N \mathbb{Z}$, and equal. Hence the conclusion follows from (2-11) and (2-12).

Remark 5.5 Numerical computations with the example of Section 6.4 show that the QHFT amplitudes should depend strongly on the choice of weight, even asymptotically. In fact, it is natural to ask whether their growth rate is determined by this choice.

Remark 5.6 (Cheeger-Chern-Simons invariants and $\mathcal{H}_{1}$ ) By the results of [24], there is an injective homomorphism from $H_{3}(\operatorname{PSL}(2, \mathbb{C}) ; \mathbb{Z})$ (discrete homology) to a scissors congruence group $\widehat{\mathcal{P}}(\mathbb{C})$. The dilogarithm (2-9), defined on $\widehat{\mathcal{P}}(\mathbb{C})$, restricts to the universal Cheeger-Chern-Simons class $\hat{C}_{2}: H_{3}(P S L(2, \mathbb{C}) ; \mathbb{Z}) \rightarrow \mathbb{C} / \pi^{2} \mathbb{Z}$, which is a constant times $\mathrm{Vol}+i C S$. Recently J Dupont and C Zickert produced dilogarithmic formulas for the lift $\widehat{C}_{2}^{\prime}$ of $\widehat{C}_{2}$ to $H_{3}(S L(2, \mathbb{C}) ; \mathbb{Z})$ [13]. In this setting $\mathcal{H}_{1}$ appears as an extension of $\exp \left(\widehat{C}_{2} / i \pi\right)$ to classes in $\widehat{\mathcal{P}}(\mathbb{C})$ representing QHFT bordisms, and coincides with $\exp \left(\hat{C}_{2}^{\prime} / i \pi\right)$ when using even flattenings. We are indebted to Dupont and Zickert [13] for pointing out the existence of such flattenings. 
Recall Definition 4.3. Assume that $\mathcal{B}^{\prime \prime}=\mathcal{B}^{\prime} * \mathcal{B}$ exists, and let $\mathcal{T}(\mathcal{B})$ and $\mathcal{T}\left(\mathcal{B}^{\prime}\right)$ be given weights $(h, k)$ and $\left(h^{\prime}, k^{\prime}\right)$, respectively. Then $\mathcal{T}\left(\mathcal{B}^{\prime}\right) * \mathcal{T}(\mathcal{B})$ is a distinguished flat/charged $\mathcal{I}$-triangulation $\mathcal{T}\left(\mathcal{B}^{\prime \prime}\right)$, with some weight $\left(h^{\prime \prime}, k^{\prime \prime}\right)$. (It follows from the Mayer-Vietoris exact sequence for $\left(\mathcal{B}^{\prime \prime}, \mathcal{B}^{\prime}, \mathcal{B}\right)$ that even if $h=h^{\prime}=0$, it can happen that $h^{\prime \prime} \neq 0$. However, if the glued part of the boundary is connected, or is a boundary in $\mathcal{B} * \mathcal{B}^{\prime}$, then $h=h^{\prime}=0$ implies $h^{\prime \prime}=0$.)

Proposition 5.7 (Functoriality) For any composition $\mathcal{B}^{\prime \prime}=\mathcal{B}^{\prime} * \mathcal{B}$ of bordisms, $\mathcal{H}_{N}\left(\mathcal{B}^{\prime \prime}, h^{\prime \prime}, k^{\prime \prime}\right)$ coincides with $\mathcal{H}_{N}\left(\mathcal{B}^{\prime}, h^{\prime}, k^{\prime}\right) \circ \mathcal{H}_{N}(\mathcal{B}, h, k)$ up to sign and multiplication by $N$-th roots of unity.

This is a direct consequence of Theorem 5.4. Also, we prove as in Proposition 4.29 of [4] that the QHFT amplitudes behave well under complex conjugation:

Proposition 5.8 (Polarity) Write $\overline{\mathcal{B}}$ for the QHFT bordism with opposite orientation and complex conjugate holonomy $\bar{\rho}$. Then $\mathcal{H}_{N}(\overline{\mathcal{B}}, h,-k)$ and $\mathcal{H}_{N}(\mathcal{B}, h, k)^{*}$, the adjoint for the hermitian structure of $V\left(\alpha_{ \pm}\right)$, coincide up to sign and multiplication by $N$-th roots of unity.

In the proof of Theorem 5.3 we have seen that the space $\widetilde{W}^{+}(T, b)$ of $(+)-\log -\mathcal{I}-$ parameters over $W^{+}(T, b)$ (the disjoint union of spaces $\left.W^{+}(T, b, \mathcal{C})\right)$ is isomorphic to $\widetilde{\mathbb{C}}^{-3 \chi(F)+2 p}$, where $\widetilde{\mathbb{C}}$ is the universal cover of $\mathbb{C} \backslash\{0\}$. Similarly, for any admissible triangulation $\tau$ of a topological cusp with $n 2$-simplices, we have the analytic subspace $\operatorname{Def}(\tau)$ of $\widehat{\mathbb{C}}^{n}$ made of the $n$-tuples of log-branches for the tetrahedra of $\mathcal{I}$-cusps with base triangulation $\tau$, where $\widehat{\mathbb{C}}$ is defined in Section 2.1. Such log-branches satisfy the compatibility relations $L_{\mathcal{T}}(e)=0$ at interior edges.

Definition 5.9 Let $X=\left(Y, L_{\mathcal{F}}, \alpha_{ \pm}\right)$be a marked topological bordism with $e-$ triangulated or admissibly triangulated boundary components. The phase space of $X$ is the (analytic) subset $\operatorname{Def}(X)$ in the product of the spaces $\widetilde{W}^{+}(T, b)$ and $\operatorname{Def}(\tau)$ over the components of $\partial \widetilde{Y}$ and $L$, determined by the family of distinguished flat/charged $\mathcal{I}$-triangulations of QHFT bordisms supported by $\left(Y, L_{\mathcal{F}}, \alpha_{ \pm}\right)$.

Proposition 5.10 (Analyticity) For every $N \geq 1$, the amplitudes of QHFT bordisms supported by $X=\left(Y, L_{\mathcal{F}}, \alpha_{ \pm}\right)$vary analytically with the boundary structure in $\operatorname{Def}(X)$, up to sign and multiplication by $N$-th roots of unity.

This follows immediately from the holomorphicity of the matrix dilogarithms, together with the fact that any path in $\operatorname{Def}(X)$ lifts to a path of log-branches, via the relations between total $\log$-branches and $\log -\mathcal{I}$-parameters (see Definition 5.2). 
When $\tilde{Y}$ is the mapping cylinder of a diffeomorphism $\phi$ of $F$, the amplitudes of QHFT bordisms supported by $\tilde{Y}$ define a morphism of the trivial vector bundle

$$
E(T, b)^{+}: \widetilde{W}^{+}(T, b) \times V(T, b) \rightarrow \widetilde{W}^{+}(T, b) .
$$

Note that any two choices of $e$-triangulations $\left(T_{1}, b_{1}\right)$ and $\left(T_{2}, b_{2}\right)$ of $F$ yield isomorphic bundles $E\left(T_{1}, b_{1}\right)$ and $E\left(T_{2}, b_{2}\right)$, with birationally equivalent bases.

When $Y$ has empty boundary, $\operatorname{Def}(X)$ is a generalization of the well known deformation space of hyperbolic structures supported by ideal triangulations of $Y \backslash L$, introduced by Thurston [30], and recently studied by Choi [12] and Champanerkar [11].

\subsection{QHFT variants}

By varying the bordism category we can vary the corresponding QFT.

QHFT $^{0}$ Consider the bordism category supported by triples $(Y, L, \rho)$, where $L$ is an nonempty unframed tangle in $Y$ and $\rho$ is a $P S L(2, \mathbb{C})$-character on the whole of $Y$ (ie $\rho$ is trivial at the meridians of $L$ ). In fact, we restrict to holonomies $\rho$ such that $(Y, L, \rho)$ admits $\mathcal{D}$-triangulations that extend a topological branched ideal triangulation $\left(T^{\prime}, b^{\prime}\right)$ of each boundary component, say $(S, V)$, and for which the link $L$ is realized as a Hamiltonian subcomplex (hence with no $\mathcal{I}$-cusp). In particular the objects of this bordism category incorporate the idealization of (necessarily idealizable) cocycles on $\left(T^{\prime}, b^{\prime}\right)$, that represent the restriction of $\rho$ to $S$. The arguments of Theorem 5.4 can be easily adapted to produce tensors $H_{N}(\mathcal{B}, h, k)$ associated to such a bordism $\mathcal{B}$, and eventually the so called QHFT $^{0}$ variant of quantum hyperbolic field theory.

When $N=1$ the $\mathrm{QHFT}^{0}$ tensors do not depend on the tangle, since the latter, being unframed, just serve to encode and guarantee the existence of global charges.

Fusion of QHFT and QHFT ${ }^{0}$ We can consider triples $\left(Y, L_{\mathcal{F}}, L^{0}, \rho\right)$, where $L=$ $L_{\mathcal{F}} \cup L^{0}$ is a tangle with a framed part $L_{\mathcal{F}}$ and an unframed one $L^{0}$. We also stipulate that $\rho$ is trivial at each meridian of $L^{0}$. For every object support $(S, V)$, we have a partition $V=V_{\mathcal{F}} \cup V_{0}$ and we use "mixed" triangulations that looks like an efficient one at $p \in V_{\mathcal{F}}$ and like an ideal one at $p \in V_{0}$. A similar mixed behavior holds for the adapted $\mathcal{D}$-triangulations of such bordisms. We eventually get tensors still denoted $\mathcal{H}_{N}(\mathcal{B}, h, k)$ giving variant functors, still denoted QHFT, that extend both the previous one $\left(L^{0}=\varnothing\right)$, and $\operatorname{QHFT}^{0}\left(L_{\mathcal{F}}=\varnothing\right)$. 
QHFT $^{e}$ Let $\left(Y, L_{\mathcal{F}}, L^{0}, \rho\right)$ be as above, and let us specialize to $\rho$ s that, as usual, are trivial at the meridians of $L^{0}$, but are not trivial at the meridians of $L_{\mathcal{F}}$. Now we use mixed triangulations of each object support $\left(S, V_{\mathcal{F}} \cup V_{0}\right)$ that look like an economic triangulation (see Remark 3.13) at each $p \in V_{\mathcal{F}}$. Concerning the adapted $\mathcal{D}$-triangulations, each component of $L_{\mathcal{F}}$ contributes to the hamiltonian subcomplex with just a copy of the parallel curve specifying the framing (recall that by using ordinary efficient triangulations, it contributed with two parallel curves). We get tensors now denoted $\mathcal{H}_{N}^{e}(\mathcal{B}, h, k)$, and a variant denoted $\mathrm{QHFT}^{e}$.

Of course, there are no deep structural differences between these variants; nevertheless each one has its own interest (see also Section 6).

\subsection{Mapping class group representations}

Fix $F$. Set $\tilde{Y}_{F}=F \times[-1,1], \tilde{L}=\partial F \times[-1,1]$ with trivial vertical framing, and let $\operatorname{Mod}(g, r)$ be the mapping class group of $F$, that is, the group of homotopy classes of orientation-preserving diffeomorphisms of $F$ fixing pointwise each boundary component. Given $\psi_{ \pm}:( \pm F,(T, b, \mathcal{L})) \rightarrow F \times\{ \pm 1\}$, put $\psi=\psi_{+}^{-1} \psi_{-}$and $[\psi]$ for the corresponding element in $\operatorname{Mod}(g, r)$. Denote by $\widetilde{W}_{[\psi]}$ the mapping torus $(F \times[-1,1]) /(x,-1) \sim(\psi(x), 1)$ of $\psi$, with tunnel boundary $\tilde{L}_{[\psi]}$. Let $\rho$ be the conjugacy class of $P S L(2, \mathbb{C})$-valued representations of $\pi_{1}(F \times[-1,1])$ (identified with $\left.\pi_{1}(F)\right)$ ) associated to $\mathcal{L}$. To simplify notation, in all statements of this section we do not mention the weights (we understand they are fixed).

Lemma 5.11 Up to sign and multiplication by $N$-th roots of unity (denoted $\left."={ }_{N} "\right)$ we have :

(1) For any fixed $(T, b, \mathcal{L})$ the amplitudes $\mathcal{H}_{N}\left(\tilde{Y}, \widetilde{L}_{F}, \rho,( \pm F,(T, b, \mathcal{L})),\left[\psi_{ \pm}\right]\right)$depend only on $[\psi]$. We denote them $\mathcal{H}_{N}([\psi])$.

(2) $\mathcal{H}_{N}([\mathrm{id}])$ is the identity map from $E_{N}\left(\alpha_{-}\right)$to $E_{N}\left(\alpha_{+}\right)$, and the composition $\mathcal{H}_{N}\left(\left[h_{2}\right]\right) \circ \mathcal{H}_{N}\left(\left[h_{1}\right]\right)={ }_{N} \mathcal{H}_{N}\left(\left[h_{2} h_{1}\right]\right)$. In particular, for any $[\psi] \in \operatorname{Mod}(g, r)$ the QHFT tensor $\mathcal{H}_{N}([\psi])$ is invertible, with inverse $\mathcal{H}_{N}\left(\left[\psi^{-1}\right]\right)$, and for a homotopically $d$-periodic $[\psi]$ the QHFT tensor $\mathcal{H}_{N}([\psi])$ is of finite order less than or equal to $d$.

(3) If $\psi(\rho)$ coincides with $\rho$, then $\operatorname{Trace}\left(\mathcal{H}_{N}([\psi])\right)={ }_{N} \mathcal{H}_{N}\left(\widetilde{W}_{[\psi]}, \widetilde{L}_{[\psi]}, \rho\right)$.

Proof Point (1) follows from Theorem 5.4, because

$$
\mathcal{H}_{N}\left(\tilde{Y}, \widetilde{L}_{F}, \rho,( \pm F,(T, b, \mathcal{L})),\left[\psi_{ \pm}\right]\right)={ }_{N} \mathcal{H}_{N}\left(\tilde{Y}, \widetilde{L}_{F}, \rho,( \pm F,(T, b, \mathcal{L})),[\mathrm{id}],[\psi]\right)
$$

(the homeomorphism $\psi_{-}^{-1} \times$ id sends the first mapping cylinder to the second). By Proposition 5.7 we have $\mathcal{H}_{N}^{2}([\mathrm{id}])={ }_{N} \mathcal{H}_{N}([\mathrm{id}])$, so $\mathcal{H}_{N}([\mathrm{id}])$ is an idempotent. It is 
invertible because the matrix dilogarithms are. Both facts imply the first claim in (2). The rest is a direct consequence of Proposition 5.7 and formula (2-22).

The arguments in the proof of Lemma 5.11 imply also that, letting $[\psi]=[\mathrm{id}]$ and $\rho$ fixed, the amplitudes of any marking variation $(T, b, \mathcal{L}) \rightarrow\left(T_{1}, b_{1}, \mathcal{L}_{1}\right)$ are invertible. Hence $\mathcal{H}_{N}([\psi])$ is conjugated to the tensor $\mathcal{H}_{N}\left(\tilde{Y}, \widetilde{L}_{F}, \rho,\left( \pm F,\left(T_{1}, b_{1}, \mathcal{L}_{1}\right)\right),\left[\psi_{ \pm}\right]\right)$. Moreover $\mathcal{H}_{N}\left(-\tilde{Y}, \widetilde{L}_{F}, \rho,(\mp F,(T, b, \mathcal{L})),\left[\psi_{ \pm}\right]\right)$, the amplitude with reversed orientation, clearly coincides with $\mathcal{H}_{N}\left([\psi]^{-1}\right)$. Using Proposition 5.8 we deduce:

Corollary 5.12 For any fixed $\rho \in \mathcal{R}(g, r)$, the homomorphisms $\psi \mapsto \mathcal{H}_{N}([\psi])$ induce a conjugacy class of linear representations of $\operatorname{Mod}(g, r)$, well-defined up to sign and multiplication by $N$-th roots of unity. For $S L(2, \mathbb{R})$-valued characters $\rho$ these representations are unitary.

\subsection{Tunneling the $(+) /(-)$ states}

We use (+)-Log-I-parameters to define the QHFT because of the existence of strongly idealizable cocycles on QHFT bordism triangulations, which makes functoriality easy to check. Here we exhibit a family of tensors correlating the $( \pm)-\log -\mathcal{I}$-parameters, thus recovering, in particular, the direct and nice interpretation of boundary structures having nontrivial holonomy at the punctures in terms of pleated hyperbolic surfaces (see Section 3.3). These tensors are also used in Section 6.3.

For any base surface $F$ with an $e$-triangulation $(T, b)$, let $Z(F)$ be the pseudomanifold obtained by collapsing to a point each boundary annulus of the cylinder $C(F)=F \times[-1,1]$. Recall the bundle $E(T, b)^{+}$in (5-1), and consider similarly $E(T, b)^{-}: \widetilde{W}^{-}(T, b) \times V(T, b) \rightarrow \widetilde{W}^{-}(T, b)$. We have:

Proposition 5.13 There exists a canonical family $\mathcal{F}$ of flat/charged $\mathcal{I}$-triangulations covering a portion of $Z(F)$, with invertible trace tensors $\mathcal{H}_{N}(\mathcal{F}): E(T, b)^{+} \rightarrow$ $E(T, b)^{-}$.

Proof Orient $C(F)$ so that $\pm F$ is identified with $F \times\{ \pm 1\}$. Let $P(T, b)$ be the cell decomposition of $C(F)$ made by the prisms with base the 2-simplices of $T$. Orient all the "vertical" (ie parallel to $[-1,1]$ ) edges of $P(T, b)$ towards $+F$. For every abstract prism $P$, every vertical boundary quadrilateral $R$ has both the two horizontal and the two vertical edges endowed with parallel orientations. So exactly one vertex of $R$ is a source (that belongs to $-F$ ), and exactly one is a pit (that belongs to $+F$ ). Triangulate each $R$ by the oriented diagonal going from the source to the pit. Finally extend the resulting triangulation of $\partial P$ to a triangulation of $P$ made of 3 tetrahedra, by taking 
the cone from the $b$-first vertex of the bottom base triangle of $P$ (note that no further vertices nor further edges have been introduced). Repeating this for every prism, we get a branched distinguished triangulation $(C(T, b), H)$ of $C(F)$, where the vertical edges make the Hamiltonian tangle $H$ (see Figure 12). As in the proof of Theorem 5.3 there exists integral charges on $(C(T, b), H)$.

Let $F \times[-1,3]$ be triangulated by two adjacent copies of $C(T, b)$, glued each to the other at $F \times\{+1\}$. For any $z \in \mathcal{Z}_{I}(T, b)^{+}$, consider the unique cocycle $C_{0}(z)$ on the composition $C(T, b) * C(T, b)$ that extends $z \cup \Psi(z)$, given on $(F \times\{-1\}) \cup(F \times\{3\})$; takes the value $P$ of (3-1) on each vertical edge contained in $F \times[1,2]$; takes the value 1 on each vertical edge contained in $F \times[-1,1]$. Perturb $C_{0}(z)$ with a 0 -cochain $s$ that takes the value 1 on $(F \times\{-1\}) \cup(F \times\{3\})$; takes values in $P B^{+}(2, \mathbb{C})$ at each vertical boundary annulus; restricts to an idealizable cocycle on $F \times[-1,1]$ and to a maximally idealizable cocycle on $F \times[1,3]$ (see Definition 3.8). Finally, glue $\mathcal{I}$-cusps to the idealization. Note that the only nonidealizable tetrahedra are those in the star of a boundary edge of the triangulation of $F \times\{3\}$. Lemma 2.13 gives flattenings for the idealizable tetrahedra.

Look at the ideal triangulation $\left(T^{\prime}, b^{\prime}\right)$ of $S \backslash V$ corresponding to the copy of the triangulation $(T, b)$ for the boundary component $F \times\{3\}$. For every cochain $s$ as above and every edge $e$ of $T^{\prime}$ we have two complex numbers: the (-)-exponential $\mathcal{I}$-parameter $W^{-}(z)(e)$, and, as in $(2-18)$, the total product $\mathcal{W}(e)$ of the cross-ratio moduli at the edges of $T$ that enter the definition of $W^{-}(z)(e)$. Recall that there are two distinct such edges only when $e$ contributes to make a marked corner. It is possible to normalize $s$ so that for every edge $e$ of $T^{\prime}$ we have $W^{-}(z)(e)=\mathcal{W}(e)^{-1}$.

Varying the cocycle $z \in \mathcal{Z}_{I}(T, b)^{+}$, this choice determines the family $\mathcal{F}$ in the statement. By perturbing the initial cocycle $z$ with 0 -cochains $t$ with values in $P S L(2, \mathbb{C}) \backslash$ $\mathrm{PB}^{+}(2, \mathbb{C})$, the same construction leads to families $\mathcal{F}_{t}$ of flat/charged $\mathcal{I}$-triangulations covering the whole of $F \times[-1,3]$.

Note that for suitable flat/charges $\left(f_{1}=0\right.$ in $(2-9)$ and $c_{1}=0$ in $\left.(2-12)\right)$ the matrix dilogarithms have well-defined finite limits when the cross-ratio modulus $w_{0} \rightarrow 0$. From the symmetry relations of the matrix dilogarithms [4, Corollary 5.6], this is true more in general for any degenerating sequence of $\mathcal{I}$-tetrahedra, that is when $w_{0}$ goes to 0,1 or $\infty$. Now, we can choose in a continuous way the flat/charges of $\mathcal{F}_{t}$ so that they satisfy the above constraints on the tetrahedra of $\mathcal{F}_{t}$ that become nonidealizable in $\mathcal{F}$, when $t \rightarrow$ id. Then $\mathcal{H}_{N}(\mathcal{F}):=\lim _{t} \mathcal{H}_{N}\left(\mathcal{F}_{t}\right)$ exists. As in Lemma 5.11 (2) we see that $\mathcal{H}_{N}\left(\mathcal{F}_{t}\right)$ is invertible, with inverse $\mathcal{H}_{N}\left(-\mathcal{F}_{t}\right)$. Since $\mathcal{H}_{N}(\mathcal{F}) \circ \mathcal{H}_{N}(-\mathcal{F})=\lim _{t}\left(\mathcal{H}_{N}\left(\mathcal{F}_{t}\right) \circ \mathcal{H}_{N}\left(-\mathcal{F}_{t}\right)\right)={ }_{N}$ id (Proposition 5.10), we deduce that $\mathcal{H}_{N}(\mathcal{F})$ is invertible. 


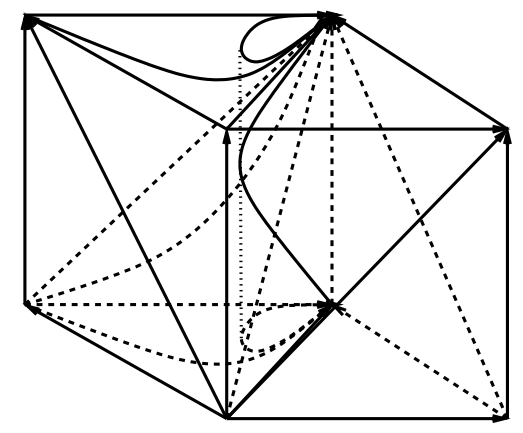

Figure 12: Pasting opposite vertical sides yields an instance of $C(T, b)$ for the once-punctured torus $S$, based on an economic triangulation of $S$ as in Remark 3.13.

\section{Partition functions}

Assume that $W$ is a closed oriented 3-manifold, and that $L$ is a link in $W$ with a framed part $L_{\mathcal{F}}$ and an unframed one $L^{0}$. Each variant of quantum hyperbolic field theory (see Section 5.3) leads to partition functions.

If $\rho$ is trivial at each meridian of $L^{0}$, we have the QHFT partition functions

$$
\mathcal{H}_{N}\left(W, L_{\mathcal{F}}, L^{0}, \rho, h, k\right)
$$

that specialize to the $\mathrm{QHFT}^{0}$ ones when $L=L^{0}$ :

$$
H_{N}(W, L, \rho, h, k)=\mathcal{H}_{N}\left(W, \varnothing, L^{0}, \rho, h, k\right) .
$$

If $\rho$ is also assumed to be nontrivial at each meridian of $L_{\mathcal{F}}$, we have also

$$
\mathcal{H}_{N}^{e}\left(W, L_{\mathcal{F}}, L^{0}, \rho, h, k\right) .
$$

These partition functions are complex-valued, well-defined up to sign and multiplication by $N$-th roots of unity. Typical examples of triples $\left(W, L_{\mathcal{F}}, \rho\right)$ are given by hyperbolic cone manifolds $W$ with framed cone locus $L_{\mathcal{F}}$ and hyperbolic holonomy $\rho$ on $W \backslash L_{\mathcal{F}}$. The partition functions can be expressed in terms of manifolds $Y$ with toric boundary and containing an unframed link $L^{0}$ in the interior. By fixing an ordered basis $\left(m_{i}, l_{i}\right)$ for the integral homology of each boundary torus, let $W$ be obtained from $Y$ by Dehn filling along the $m_{i}$, and $L_{\mathcal{F}}$ be the disjoint union of the cores of the filling solid tori, framed by the $l_{i}$. Then the partition functions of $\left(W, L_{\mathcal{F}}, L^{0}, \rho, h, k\right)$ are in fact invariants of $\left(Y,\left\{\left(m_{i}, l_{i}\right)\right\}_{i}, L^{0}, \rho, h, k\right)$. 
When $N=1$ the $\mathrm{QHFT}^{0}$ partition functions $H_{1}(W, L, \rho, h, k)$ do not depend on $L$, and coincide with the simplicial formulas obtained in [24] for the Cheeger-ChernSimons invariants of pairs $(W, \rho)$ (see [4], where the weights were normalized as $0)$.

In the rest of this section we concentrate on the case $N>1$.

\subsection{QHFT vs QHFT $^{0}$ partition functions}

For $\mathcal{B}=(W, L, \rho), L=L^{0}$, with weights $h=k=0, H_{N}(W, L, \rho, 0,0)$ coincide with the invariants $H_{N}(W, L, \rho)$ constructed in [3; 4]. Let us consider more generally $\left(W, L_{\mathcal{F}}, L^{0}, \rho, 0,0\right)$. Fix also a framing $\mathcal{F}_{0}$ for $L^{0}$. Then we can consider the partition function $\mathcal{H}_{N}\left(W, L_{\mathcal{F}} \cup L_{\mathcal{F}_{0}}^{0}, \varnothing, \rho, 0,0\right)$. Let us denote by $\bar{\lambda}$ the unframed link obtained by splitting each component of $L^{0}$ in the two corresponding parallel boundary components of the ribbon link $L_{\mathcal{F}_{0}}^{0}$. We have:

\section{Proposition 6.1}

$$
\mathcal{H}_{N}\left(W, L_{\mathcal{F}} \cup L_{\mathcal{F}_{0}}^{0}, \varnothing, \rho, 0,0\right)={ }_{N} \mathcal{H}_{N}\left(W, L_{\mathcal{F}}, \bar{\lambda}, \rho, 0,0\right) .
$$

Proof For simplicity, assume that $L=L^{0}$. Fix a $\mathcal{D}$-triangulation of $\left(\widetilde{W}, \widetilde{L}_{\mathcal{F}}, \rho\right)$ where each tunnel component $B$ has a symmetric admissible triangulation as in Figure 13 (opposite sides of the quadrilateral are identified). The tangle $\bar{\lambda}$ cuts open $B$ into symmetric annuli, left and right to the central vertical line in Figure 13.

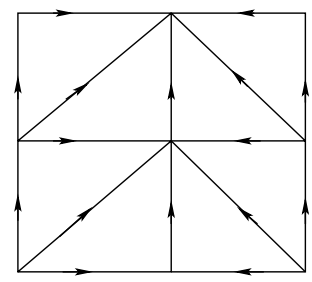

Figure 13: A special admissible triangulation of $B$

Because $\rho$ has trivial holonomy at the meridians of $L_{\mathcal{F}}$, we can assume that the cocycle takes the same values on symmetric edges. Identifying the annuli we thus get a $\mathcal{D}$-triangulation for the $\mathrm{QHFT}^{0}$ triple $(W, \bar{\lambda}, \rho)$. Since $H_{N}(W, \bar{\lambda}, \rho)$ is computed from the idealization and symmetric tetrahedra in the cusps have opposite branching orientation, the result will follow if we show the existence of symmetric flat/charges. Then each cusp tensor will be the identity map. 
The existence of flattenings with this property can be shown by using Lemma 2.13, but for charges we need to take another route. Recall from the end of Section 2 that flat/charges form affine spaces over an integral lattice generated by vectors attached to the edges. For an edge of $B$, such vectors can be represented as adding +1 at one of the left adjacent corner and -1 at the other, and the inverse for the right adjacent corners. Using these rules and (2-20) it is straightforward (though tedious) to check that any given flat/charge can be turned into one with equal quantum log-branches on symmetric tetrahedra.

\subsection{Invariants of cusped manifolds and surgery formulas}

Let us recall the QHG pseudomanifold triangulations $\mathcal{T}$ used in [4, Definition 6.2 and Definition 6.3] to define the quantum hyperbolic invariant trace tensors $\mathcal{H}_{N}(\mathcal{T})$ for oriented cusped hyperbolic manifolds.

Let $M$ be a cusped manifold. Denote by $Z$ the pseudomanifold obtained by taking the one point compactification of each cusp of $M . M$ admits a triangulation by positively embedded hyperbolic ideal tetrahedra, possibly including some degenerate ones of null volume (ie having real cross-ratios). Such a triangulation can be obtained by subdividing the canonical Epstein-Penner cell decomposition of $M$. This gives rise to triangulation $\left(T_{0}, z_{0}\right)$ of $Z$, where $z_{0}$ is the cross-ratio function of the abstract edges of $T$, the imaginary part of every cross-ratio being $\geq 0$. We call it a quasi-geometric ideal triangulation of $Z$. If some quasi-geometric triangulation admits a global branching, we say that $M$ is gentle. More generally, $M$ is said weakly-gentle if there is an $\mathcal{I}$-triangulation $(T, b, w)$ of $Z$ such that $(T, z), z=w^{* b}$, is obtained via a (possibly empty) finite sequence $\left(T_{0}, z_{0}\right) \rightarrow \ldots \rightarrow\left(T_{i}, z_{i}\right) \rightarrow \ldots \rightarrow(T, z)$ of positive $2 \rightarrow 3$ transits, where $\left(T_{0}, z_{0}\right)$ is a quasi-geometric triangulation of $Z$ as above. Each transit $\left(T_{i}, z_{i}\right) \rightarrow\left(T_{i+1}, z_{i+1}\right)$ is defined by $W_{T_{i}}(e)=W_{T_{i+1}}(e)$, with all exponents $*_{b}=1$ (see $(2-18))$. Every such a $(T, b, w)$ can be enhanced to flat/charged $\mathcal{I}$-triangulations $\mathcal{T}=(T, b, w, f, c)$.

Remark 6.2 Canonical flattenings can be defined only if $(T, b, w)$ is obtained from the idealization procedure (see Definition 2.11). This should happen very rarely, since ideal triangulations with few vertices carry few idealizable cocycles. For instance, there is no such cocycle for the canonical triangulation of the figure eight knot complement.

Given $\left(T_{0}, z_{0}\right)$, it is certainly possible to get an $\mathcal{I}$-triangulation $(T, b, w)$ by performing also some bubble moves (hence introducing new interior vertices). The authors do not know any example of nonweakly-gentle cusped manifold, that is, such that we are forced to do it. Anyway, dealing with bubble moves is a technical difficulty which 
will appear also in the proof of Theorem 6.4 (1) below. We overcome it as follows. We fix an edge $a$ of the canonical Epstein-Penner cell decomposition of $M$, and take $A$ made by two copies of $a$ that intersect at nonmanifold points of $Z$; the second copy runs parallel to $a$ within an open cell of the decomposition. Hence, $A$ is a circle covered by two arcs. We need to enlarge the notions introduced in Definition 2.17. We say that $(T, H)$ is a distinguished triangulation of $(Z, a)$ if $H$ is a subcomplex of the 1 -skeleton of $T$ isotopic to $A$, that contains all the regular vertices of $T$, and such that one arc of $A$ is covered by an edge $l$ of $H$. We say that $c$ is a global charge on $(T, H)$ if

$$
C_{\mathcal{T}}(e)= \begin{cases}4 & \text { if } e=l \\ 0 & \text { if } e \subset H \backslash l \\ 2 & \text { if } e \subset T \backslash H\end{cases}
$$

If $H=\varnothing$ this reduces to the usual notion of global charge on a closed triangulated pseudomanifold whose nonmanifold points have toric links. By using bubble moves and the existence of such usual global charges, it is easily seen that $(T, H)$ supports global charges as in (6-1) (see the proof of Theorem 6.8 in [4] for the details).

We say that $\mathcal{T}=(T, H, b, w, f, c)$ is a flat/charged $\mathcal{I}$-triangulation of $(Z, a)$ if $(T, H, b, c)$ is a branched, charged and distinguished triangulation of $(Z, a)$, and $(T, z), z=w^{*_{b}}$, is obtained from a quasi-geometric $\left(T_{0}, z_{0}\right)$ via a finite sequence $\left(T_{0}, z_{0}\right) \rightarrow \ldots \rightarrow(T, z)$ of transits supported by positive $2 \rightarrow 3$ moves and bubble moves. By setting $a=\varnothing$ and $H=\varnothing$, this definition incorporates that for the weaklygentle case.

In [4] it is shown that flat/charged $\mathcal{I}$-triangulations $\mathcal{T}$ of $(Z, a)$ (with arbitrary weights) do exist and that

$$
H_{N}(M, a)=\mathcal{H}_{N}(\mathcal{T})
$$

is a well defined invariant of $(M, a)$, providing the weights of flat/charges to be 0 . To simplify the exposition, below we continue with this normalization. When $M$ is weakly-gentle we get invariants $H_{N}(M)$. In fact, as a by-product of the following discussion, we will realize that $H_{N}(M, a)$ does not depend on the choice of $a$, so that $H_{N}(M)$ is always well defined (see Corollary 6.5).

Let us recall now a few facts related to hyperbolic Dehn filling [30; 7; 27]. A quasigeometric triangulation $\left(T_{0}, z_{0}\right)$ as above corresponds to the complete structure of $M$. It can be deformed in a complex variety of dimension equal to the number of cusps. If $z^{\prime}$ is close enough to $z_{0},\left(T_{0}, z^{\prime}\right)$ is a triangulation by (possibly negative [27]) embedded hyperbolic ideal tetrahedra in a noncomplete hyperbolic structure, say $M^{\prime}$, close to $M$. In some case the completion of $M^{\prime}$ gives rise to a compact closed 
hyperbolic manifold $W$, topologically obtained by Dehn filling of the truncated cusps of $M$. The core of each attached solid torus is a "short" simple closed geodesic $L_{j}$ of $W$, so that we have the geodesic link $L=\bigsqcup_{j} L_{j}$. Moreover, there are sequences ( $W^{n}, L^{n}$ ) obtained in this way such that the length of $L^{n}$ goes to 0 when $n \rightarrow+\infty$. Hence $\left(W^{n}, L^{n}\right)$ converges to the cusped manifold $M$ in a neat geometric sense. From now on we will consider small deformations $z^{\prime}$ leading to such closed completions.

As well as $\left(T_{0}, z_{0}\right)$ gives rise to a triangulation $\mathcal{T}=(T, H, b, w, f, c)$ of $(Z, a), z^{\prime}$ close to $z_{0}$ gives rise to another flat/charged $\mathcal{I}$-triangulation $\mathcal{T}^{\prime}=\left(T, H, b, w^{\prime}, f^{\prime}, c\right)$, where $w^{\prime}$ is close to $w$ and the log-branch associated to the global flattening $f^{\prime}$ corresponds to a continuous deformation of the one for $f$. Recall that we have normalized the flattening weights as 0 .

Lemma 6.3 (See Neumann [24, p 469].) Let $z^{\prime}$ be a small deformation of $z_{0}$ producing $(W, L)$, and $m_{j}$ be a meridian of each link component $L_{j}$. Then there exist flattenings $f^{\prime \prime}$ for the deformed triangulation $\left(T, b, w^{\prime}\right)$ such that the weights $\gamma_{2}\left(f^{\prime \prime}\right)$ and $\gamma\left(f^{\prime \prime}\right)$ of $\mathcal{T}^{\prime \prime}=\left(T, H, b, w^{\prime}, f^{\prime \prime}, c\right)$ satisfy $\gamma_{2}\left(f^{\prime \prime}\right)=0$ and $\gamma\left(f^{\prime \prime}\right)\left(m_{j}\right)=0$ for all $j$.

Note that the new weight $\gamma\left(f^{\prime \prime}\right)$ is not completely determined, and that for any framing curve $l_{j}$ of $L_{j}$ (ie a longitude of a tubular neighborhood of $L_{j}$ ) the deformed weight is of the form $\gamma\left(f^{\prime}\right)\left(l_{j}\right)=\lambda\left(L_{j}\right)+2 k^{\prime} \pi \sqrt{-1}, k^{\prime} \in \mathbb{Z}$, where $\lambda\left(L_{j}\right)$ is the complex length of $L_{j}$, that is, the logarithm of the dilation factor of its holonomy, which is a loxodromic transformation of $\mathbb{H}^{3}$. Then we can take any value $\gamma\left(f^{\prime \prime}\right)\left(l_{j}\right)=\lambda\left(L_{j}\right)+2 k^{\prime \prime} \pi \sqrt{-1}$, $k^{\prime \prime} \in \mathbb{Z}$.

Proof The logarithm of the derivative of the holonomy of $m_{j}$ is 0 at the complete structure, but after deformation it represents a full $2 \pi$-rotation about $L_{j}[30 ; 7]$. Hence $\gamma\left(f^{\prime}\right)\left(m_{j}\right) \in \mathbb{Z} \sqrt{-1} \pi$. The result then follows from the comments after Theorem 2.20.

An explicit construction of flattenings $f^{\prime \prime}$ as in the lemma shall be recalled during the proof of Theorem 6.4. Hence, we dispose of two flat/charged $\mathcal{I}$-triangulations of $(Z, a), \mathcal{T}^{\prime}=\left(T, H, b, w^{\prime}, f^{\prime}, c\right)$ and $\mathcal{T}^{\prime \prime}=\left(T, H, b, w^{\prime}, f^{\prime \prime}, c\right)$, relative to a small deformation $z^{\prime}$ of the complete structure $z_{0}$ as above, leading to respective trace tensors $\mathcal{H}_{N}\left(\mathcal{T}^{\prime}\right)$ and $\mathcal{H}_{N}\left(\mathcal{T}^{\prime \prime}\right)$. By the comments above, the boundary weight of the triangulation $\mathcal{T}^{\prime \prime}$ is not completely determined. However we have:

Theorem 6.4 (Cusped manifold surgery formula) Let $(W, L)$ be obtained by completion of a small deformation $z^{\prime}$ of $z_{0}$, and $\mathcal{T}^{\prime}, \mathcal{T}^{\prime \prime}$ be associated triangulations. 
Denote by $\rho$ the hyperbolic holonomy of $W$. Then we have $H_{N}(W, L, \rho)={ }_{N}$ $\mathcal{H}_{N}\left(\mathcal{T}^{\prime \prime}\right)$. Moreover, associated to each cusp $C_{j}$ of $M$ there is an explicitly known map $\Lambda_{N}^{j}\left(\mathcal{T}^{\prime \prime}\right):\{N-$ states of $\mathrm{T}\} \rightarrow \mathbb{C}$ such that the following surgery formula holds:

$$
H_{N}(W, L, \rho)={ }_{N} \sum_{s} \prod_{\Delta \subset T} \mathcal{R}_{N}\left(\Delta, b, w^{\prime}, f^{\prime}, c\right)_{s} \prod_{j} \Lambda_{N}^{j}\left(\mathcal{T}^{\prime \prime}\right)(s)
$$

where $s$ runs over the $N$-states of $T$ and $\mathcal{R}_{N}\left(\Delta, b, w^{\prime}, f^{\prime}, c\right)_{s}$ is the matrix dilogarithm entry determined by $s$, for the tetrahedron with the continuously deformed structure.

Corollary 6.5 If $\left\{\left(W_{n}, L_{n}, \rho_{n}\right)\right\}$ is a sequence of closed hyperbolic Dehn fillings converging to the cusped manifold $M$, then for every arc $a, \lim _{n} H_{N}\left(W_{n}, L_{n}, \rho_{n}\right)={ }_{N}$ $H_{N}(M, a)$. Hence $H_{N}(M)=H_{N}(M, a)$ is always a well defined invariant of $M$ (beyond the weakly-gentle case).

Proof Take the triangulations $\mathcal{T}_{n}^{\prime \prime}$ associated as in Theorem 6.4 to $\left(W_{n}, L_{n}, \rho_{n}\right)$ so that $\gamma\left(f_{n}^{\prime \prime}\right)\left(\left(l_{j}\right)_{n}\right)=\lambda\left(\left(L_{j}\right)_{n}\right)$. Since this goes to 0 when $n$ goes to infinity, the limit triangulation of the sequence $\left\{\mathcal{T}_{n}^{\prime \prime}\right\}$ has 0 flattening weights. Since the state sum $H_{N}(\mathcal{T})$ is an analytic function of the log-branches, the result follows.

Remarks 6.6 (1) Theorem 6.4 is the analog for $N>1$ of Theorems 14.7 and Theorem 14.5 in [24], which describe surgery formulas for the volume, $\operatorname{Vol}(W)$, and Chern-Simons invariant, $\mathrm{CS}(W)$, of $W$ :

$$
\sqrt{-1}(\operatorname{Vol}(W)+\sqrt{-1} \operatorname{CS}(W))=\sum_{\Delta \subset T} \mathcal{R}\left(\Delta, b, w^{\prime}, f^{\prime}\right)-\frac{\pi \sqrt{-1}}{2} \sum_{j} \lambda\left(L_{j}\right)
$$

where $\mathcal{R}$ is given by (2-9) and $\lambda\left(L_{j}\right)$ is the complex length of $L_{j}$ (see the comments after Lemma 6.3). The technical complications due to the bubble moves disappear for $N=1$.

(2) If $M$ is gentle and has a geometric branched ideal triangulation $(T, b, w)$ without degenerate tetrahedra, then for each 3 -simplex the flattenings of $\mathcal{T}^{\prime}$ for a sufficiently small deformation are just $-*_{b}$ times integral charges. It follows from the proof of Theorem 6.4 that the scissors congruence class $\mathfrak{c}_{\mathcal{I}_{f c}}(W, L, \rho)$ of [4, Section 7] coincides with Neumann's deformed scissors congruence class $\hat{\beta}\left(M^{\prime}\right)$ in [24, Theorem 14.7] (see also Remark 6.12 and Conjecture 7.9 in [4], where the undeformed $\hat{\beta}(M)$ is denoted $\left.\mathfrak{c}_{\mathcal{I}_{f c}}(M)\right)$.

(3) In general there are small deformations $z$ of $z_{0}$ leading to complete manifolds that are still cusped, that is only some cusps of $M$ have supported a hyperbolic Dehn 
filling. There are also sequences of such cusped manifolds $M^{n}$, with (short) geodesic links $L^{n}$, converging to $M$. Similarly to the fusion of QHFT with QHFT $^{0}$ (see Section 5.3) we can define quantum hyperbolic invariants $H_{N}\left(M^{n}, L^{n}\right)$ for which the natural extensions of Theorem 6.4 and Corollary 6.5 hold.

(4) Theorem 6.4, as well as Theorem 6.7 below, shows a crucial distinction between flattenings and charges in surgery, since we have to vary the former. This is explicated in the example of Section 6.4.

Let us consider now $\left(W, L_{\mathcal{F}}, L^{0}, \rho\right)$. Let $L_{j}$ be a component of $L_{\mathcal{F}}, \bar{\lambda}_{j}=L_{j} \cup L_{j}^{\prime}$, $L_{j}^{\prime}$ being the longitude of $L_{j}$ specifying the framing. Let $U=U\left(L_{j}\right)$ be a tubular neighborhood of $L_{j}$ in $W$, and $l \subset \partial U$ be a nonseparating simple closed curve. Let $W(l)$ be obtained from $W$ by the Dehn filling of $W \backslash \operatorname{Int}(U)$ along $l$. Denote by $l^{*}$ the core of the attached solid torus.

Theorem 6.7 (Closed manifold surgery formula) Assume that $\rho(l)=\mathrm{id} \in \operatorname{PSL}(2, \mathbb{C})$ and the weight $k$ satisfies $k([l])=0$. Denote by $\rho^{\prime}$ the natural extension of $\rho_{\mid W \backslash U}$ to $W(l) ; \tilde{L}_{\mathcal{F}}=L_{\mathcal{F}} \backslash L_{j} ; k^{\prime}$ the restriction of $k$ to $W(l)$. We have

$$
\mathcal{H}_{N}\left(W, L_{\mathcal{F}}, L^{0}, \rho, 0, k\right)={ }_{N} \mathcal{H}_{N}\left(W(l), \tilde{L}_{\mathcal{F}}, L^{0} \cup l^{*} \cup \bar{\lambda}_{j}, \rho^{\prime}, 0, k^{\prime}\right) .
$$

If moreover $\rho$ is not trivial at the meridians of $L_{\mathcal{F}}$ and $l$ is a longitude of $\partial U$, then

$$
\mathcal{H}_{N}^{e}\left(W, L_{\mathcal{F}}, L^{0}, \rho, 0, k\right)={ }_{N} \mathcal{H}_{N}^{e}\left(W(l), \tilde{L}_{\mathcal{F}}, L^{0} \cup l^{*} \cup L_{j}^{\prime}, \rho^{\prime}, 0, k^{\prime}\right) .
$$

Let us assume now that $L^{0}$ is made by $r$ parallel copies of $L_{j}$ along the ribbon $L_{\mathcal{F}}$ that encodes the framing. So denote $L^{0}$ by $\lambda_{r}$; with this notation, $\bar{\lambda}=\lambda_{2}$. Assume furthermore that $l=m$ is a meridian of $L_{j}$, so that $l^{*}=L_{j}$. By applying inductively both (6-2) and Proposition 6.1 to this situation we get the following:

Corollary 6.8 For every $r \geq 2$ we have

$$
H_{N}\left(W, \lambda_{r}, \rho\right)={ }_{N} H_{N}\left(W, \lambda_{2}, \rho\right) .
$$

Remarks 6.9 (1) Though disjoint and complementary by hypothesis, formula (6-3) is formally the same as that of Proposition 6.1, when replacing $l$ by $m$.

(2) Assume (for simplicity) that $L=L_{\mathcal{F}}$. When $l$ is a longitude of $L_{j}, l^{*}$ inherits a natural framing in $W(l)$. Hence we get a triple $\left(W(l), \hat{L}_{\widehat{\mathcal{F}}}, \rho\right)$. It follows from the very definition of the QHFT tensors that $\mathcal{H}_{N}\left(W, L_{\mathcal{F}}, \rho, 0,0\right)=\mathcal{H}_{N}\left(W(l), \widehat{L}_{\hat{\mathcal{F}}}, \rho, 0,0\right)$ and the same with $\mathcal{H}_{N}^{e}$ (when defined) replacing $\mathcal{H}_{N}$. 
(3) We have seen that both $\mathcal{H}_{N}$ and $\mathcal{H}_{N}^{e}$ partitions functions display interesting features of QHG. A main advantage of the $\mathcal{H}_{N}$ ones is the possibility to set in a same "holomorphic family" the QHG tensors associated to characters that are both trivial and nontrivial at link meridians. Consider for example a hyperbolic knot $L$ in $S^{3}$, endowed with the canonical framing $\mathcal{F}$. Kashaev's volume conjecture concerns the asymptotic behavior of $H_{N}\left(S^{3}, L, \rho_{\text {triv }}\right)$ when $N \rightarrow+\infty$. A reasonable variant of it is in terms of the partition functions $\mathcal{H}_{N}\left(S^{3}, L_{\mathcal{F}}, \rho_{\text {triv }}, 0,0\right)={ }_{N} H_{N}\left(S^{3}, \bar{\lambda}, \rho_{\text {triv }}\right)$. A family as above could be useful in order to establish connections with the $\mathcal{H}_{N}$ partition functions of $\left(S^{3}, L_{\mathcal{F}}, \rho_{\text {hyp }}\right)$, where $\rho_{\text {hyp }}$ is the hyperbolic holonomy of the cusped manifold $M=S^{3} \backslash L$ (see Remark 6.14).

The rest of the section is devoted to the proof of these results. This goes in several steps.

A main tool is the simplicial blowing up/down procedure considered by Neumann in [24, Section 11]. We use it just to get a simplicial version of (topological) Dehn filling. Let $Z$ be a pseudomanifold without boundary such that every nonmanifold point has toric link. Let $v$ be a nonmanifold point. Consider a closed cone neighborhood $N(v)$ of $v$, and a nonseparating simple closed curve $C$ on the torus $\partial N(v)$. The topological Dehn filling of $Z$ at $v$ along $C$ is the pseudomanifold $Z^{\prime}$ obtained by gluing a 2-handle to $Z \backslash \operatorname{Int}(N(v))$ along $C$, and then collapsing to one point the resulting boundary component.

Now, let $T$ be a pseudomanifold triangulation of $Z$. Consider the abstract star $\operatorname{Star}^{0}(v)$ of $v$ in $T$. The boundary of $\operatorname{Star}^{0}(v)$ is the abstract link $\operatorname{Link}(v)$ which is homeomorphic to $\partial N(v)$. Assume that the curve $C$ is realized as a simplicial curve on $\operatorname{Link}(v)$. Then the cone from $v$ over $C$ in $\operatorname{Star}^{0}(v)$ is a triangulated disk $D^{0}$. The interior of $\operatorname{Star}^{0}(v)$ embeds onto the interior of the actual star of $v$ in $T, \operatorname{Star}(v)$, which is made of the union of the 3 -simplices having $v$ as a vertex. In this way $D^{0}$ maps onto a triangulated singular disk $D$ in $Z$, that has embedded interior and singular boundary immersed in the boundary of $\operatorname{Star}(v)$. Cut open $T$ along $\operatorname{Int}(D)$ and glue the double cone $C D$ of $D$ (this is a triangulated singular 3-ball Figure 14) so that the top and the bottom get identified with the two copies of $D$ resulting from slicing. This gives a triangulation $T^{\prime}$ of the pseudomanifold $Z^{\prime}$ obtained by Dehn filling along $C$. It has the property that every (abstract) tetrahedron of $T$ persists in $T^{\prime}$. Referring to the topological description, the interior of the cocore of the 2-handle attached to $Z \backslash \operatorname{Int}(N(v))$ is isotopic to the interior of the union $H^{\prime}$ of two edges, each joining $v$ to the new vertex $v^{\prime}$ at the "center" of $C D$. In fact $H^{\prime}$ is the core of the solid torus added by the Dehn filling. 
Remark 6.10 Note that in general there are very few simplicial curves on a given $\operatorname{Link}(v)$. Hence to get such a simplicial description of an arbitrary Dehn filling, we will usually have to modify a given triangulation. For the peculiar QHG pseudomanifold triangulations considered in this section, retriangulating will be possible by using QHG isomorphisms, hence without altering the trace tensors. In fact, any two triangulations of $\partial N(v)$ are connected by a finite sequence of 2 -dimensional $1 \leftrightarrow 1$ "flip" moves (see Figure 6), and $1 \leftrightarrow 3$ moves obtained by replacing a 2 -simplex with the cone of its boundary to a point. Since $N(v)$ is homeomorphic to $\operatorname{Link}(v)$, any such a sequence is the boundary trace of a sequence of $2 \leftrightarrow 3$ moves and bubble moves in $\operatorname{Star}(v)$. (Note, in particular, that $H$ passes through the new interior vertices). By using the arguments of Theorem 6.8 in [4], we will always be able to choose that sequence so that it lifts to a sequence of QHG transits.

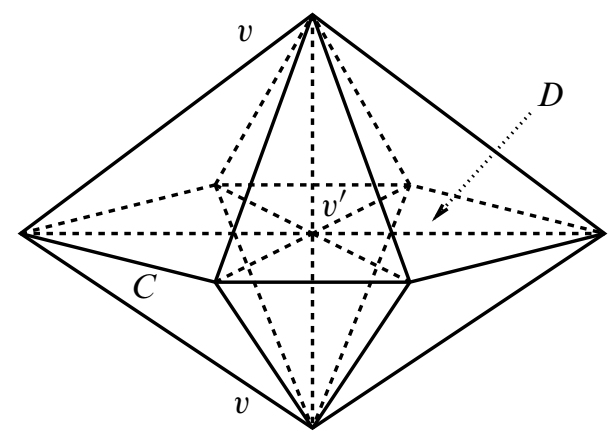

Figure 14: An instance of double cone on a disc

Let us consider now a distinguished triangulation $(T, H)$ of $(Z, a)$ as above, and let $Z^{\prime}$, triangulated by $T^{\prime}$, be the result of a simplicial Dehn filling of $Z$ along a curve $C$. Denote $H^{\prime \prime}$ the graph union of the knot $H^{\prime}$ (the core of the solid torus) and the image of $H$ in $T^{\prime}$. We define the notion of global charge on $\left(T^{\prime}, H^{\prime \prime}\right)$ by formally replacing $H$ by $H^{\prime \prime}$ in (6-1).

Lemma 6.11 Let $c$ be a global charge on $(T, H)$ such that the charge weight of the curve $C$ is 0 . Then $c$ extends to a global charge $c^{\prime}$ on $\left(T^{\prime}, H^{\prime}\right)$.

Proof The complex $C D$ is made of pairs of adjacent 3-simplices, respectively above and below the disk $D$. For a 3 -simplex of the top layer with charges $c_{0}$ and $c_{1}$ at the edges in $D$ (ordered by using an orientation of $D$, say), we will put the charges $-c_{0}$ and $2-c_{1}$ at these edges for the symmetric 3 -simplex in the bottom layer. Then the 
other charges are $c_{2}=1-c_{0}-c_{1}$ and $-c_{2}$, respectively. We have the charge sum $C_{T^{\prime}}(e)=2$ at each interior edge of $D$, and $C_{T^{\prime}}(e)=C_{T}(e)$ at the edges $e$ of $\partial D$. For the top edges $e^{\prime}$ of $C D$ we can also choose the charges so that $C_{T^{\prime}}\left(e^{\prime}\right)$ equals $C_{T}(e)$, where $e$ is the copy of $e^{\prime}$ in $D \subset M$. Indeed, there are $n$ degrees of freedom in doing this, where $n$ is the number of 1 -simplices in the curve $C$ used for blowing down. Then we check that $C_{T^{\prime}}\left(e^{\prime}\right)=2$ at the bottom edges. In particular, the subcomplex $H$ survives in $T^{\prime}$.

Note that $C_{T^{\prime}}\left(e_{0}\right)=-C_{T^{\prime}}\left(e_{1}\right)$ at the edges $e_{0}$ and $e_{1}$ of $H^{\prime}$. We have to check that $C_{T^{\prime}}\left(e_{0}\right)=0$, so that $(2-25)$ is satisfied on $\left(T^{\prime}, H^{\prime \prime} \backslash l\right)$. In fact, $C_{T^{\prime}}\left(e_{0}\right)$ is $n$ minus the sum of the $2 n$ charges at the bottom edges of $C D$, which is also the sum of charges in $T^{\prime} \backslash C D$ at these edges, minus $n$. We can form $n$ pairs of such charges corresponding to the 3-simplices of the ideal triangulation $T$ of $M$ having a 2-simplex in $D$. Replacing for each of them the pair with 1 minus the last charge, we get that $C_{T^{\prime}}\left(e_{0}\right)$ is equal to $\gamma(a)$, with $\gamma$ defined in Section 2 and $a$ is a normal path in $\operatorname{Link}(v)$ that runs parallel to $C$ on one side (see Figure 15). Because the weight of $C$ is zero, we deduce $C_{T^{\prime}}\left(e_{0}\right)=0$.

If $H \neq \varnothing$, we have to show that it can be deleted from $H^{\prime \prime}$. As the two components $l$ and $H \backslash l$ are isotopic and satisfy $C_{T^{\prime}}(l)=4$ and $C_{T^{\prime}}(e)=0$ for each edge $e \in H \backslash l$, we can retriangulate the surgered pseudomanifold $Z^{\prime}$ so as to delete them, by using a sequence of charge transits starting from $\left(T^{\prime}, H^{\prime \prime}\right)$ and terminating with a negative bubble move [3, Proposition 4.27; 4, proof of Theorem 6.8]. Retriangulating $Z^{\prime}$ backward, we eventually find a sequence of charge transits terminating at $\left(T^{\prime}, H^{\prime}, c^{\prime}\right)$. The result follows.

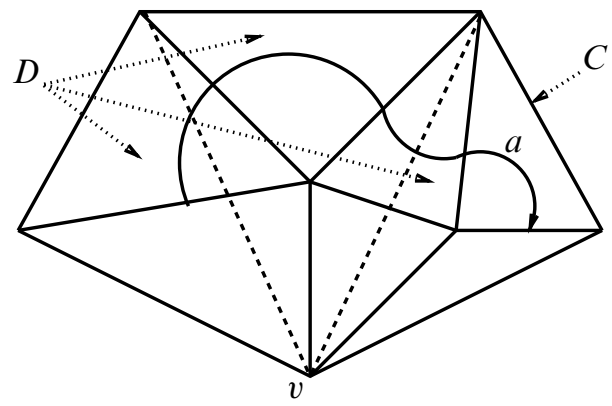

Figure 15: A normal path $a$ in $\operatorname{Link}(v)$ running parallel to a blowing-up curve $C$. Four 3-simplices of $T^{\prime} \backslash C D$ glued to three 2-simplices of $D$ are shown in the picture. 
Proof of Theorem 6.4 To simplify assume that $M$ has only one cusp. Take $\mathcal{T}^{\prime \prime}=$ $\left(T, H, b, w^{\prime}, f^{\prime \prime}, c\right)$. For the first claim we assume that the meridian $m$ of $L$ is a simplicial path in $\operatorname{Link}(v)$, where the vertex $v$ of $T$ corresponds to the filled cusp. This is possible due to Remark 6.10.

Lemma 6.11 implies that $c^{\prime}$ extends to $\left(T^{\prime}, H^{\prime}\right)$ after the Dehn filling along $m$. Extend the branching $b$ by letting the new vertex $v^{\prime}$ be a pit of the double cone $C D$ we splice in $T$. By using Lemma 6.3, arguments similar to that of Lemma 6.11 show that we can give the same log-branches on the 3-simplices of $C D$, in a pair above and below the disk $D$ [24, p 454]. Hence we get a distinguished flat/charged $\mathcal{I}$ triangulation for $(W, L, \rho)$. The weight $h \in H^{1}(W ; \mathbb{Z} / 2 \mathbb{Z})$ is clearly 0 because of the epimorphism $H_{1}(M ; \mathbb{Z} / 2 \mathbb{Z}) \rightarrow H_{1}(W ; \mathbb{Z} / 2 \mathbb{Z})$ induced by inclusion. As in the first claim of Lemma 5.11 (2) we see that the (unnormalized) trace tensor for $C D$ is $N$ times the identity map from the linear space attached to the top copy of $D$ to that for the bottom one. Combining this with the normalization of trace tensors in (2-22) gives $H_{N}(W, L, \rho)=\mathcal{H}_{N}\left(\mathcal{T}^{\prime \prime}\right)$

By Lemma 6.3 we have $\left(\gamma\left(f^{\prime \prime}\right)-\gamma\left(f^{\prime}\right)\right)(m)=-2 \sqrt{-1} \pi$. Hence the collection of values of $f^{\prime \prime}-f^{\prime}$ determines a path $l$ normal to the cusp triangulation induced by $T$, that intersects $m$ once and whose homology class is Poincaré dual to $\left(\gamma\left(f^{\prime \prime}\right)-\right.$ $\left.\gamma\left(f^{\prime}\right)\right) / 2 \sqrt{-1} \pi$. Denote by

$$
\Delta\left(l_{j}\right)=\left(\Delta^{1}, \ldots, \Delta^{\left|\Delta\left(l_{j}\right)\right|}\right)
$$

the sequence of flat/charged $\mathcal{I}$-tetrahedra (possibly with repetitions) determined by the 2 -simplices met by $l$. Each time $l$ goes through a $2-$ simplex it selects one of its vertices, whence a cross-ratio modulus, say $z_{i}$, of the tetrahedron $\Delta^{i}$ corresponding to the 2 -simplex. The values of $f^{\prime \prime}$ on $\Delta^{i}$ are obtained from those of $f^{\prime}$ by adding or subtracting 1 at the edges corresponding to the other two vertices, as indicated in Figure 16. For any fixed tetrahedron $\Delta$ of $T$ all three flattenings may be eventually altered. They differ from those of $f^{\prime}$ by adding or subtracting integers distinct from $-1,0$ or 1 exactly when $\Delta=\Delta^{i}=\Delta^{j}$ for some $i \neq j$. Now, recall from (2-22) that

$$
H_{N}(M, a)=\mathcal{H}_{N}\left(\mathcal{T}^{\prime}\right)=\sum_{s} \prod_{\Delta \subset T} \mathcal{R}_{N}(\Delta, b, w, f, c)_{s}
$$

Put $\zeta=\exp (2 \pi \sqrt{-1} / N)$. For any $x \in \mathbb{C} \backslash\left\{\zeta^{j}, j=1, \ldots, N-1\right\}$ the function $g$ defined in (2-10) satisfies [4, Lemma 8.2]:

$$
g\left(x \zeta^{k}\right)=g(x) \prod_{j=1}^{k} \frac{\left(1-x^{N}\right)^{1 / N}}{1-x \zeta^{j}} .
$$

Algebraic $8 \mathcal{G}$ Geometric Topology, Volume 7 (2007) 
Then, it is easily checked that for a flat/charged $\mathcal{I}$-tetrahedron $\left(\Delta, b, w^{\prime}, f^{\prime}, c\right)$ with $f^{\prime}=\left(f_{0}^{\prime}, f_{1}^{\prime}, f_{2}^{\prime}\right)$ and positive branching orientation, if $f^{\prime \prime}=\left(f_{0}^{\prime}+n, f_{1}^{\prime}, f_{2}^{\prime}-n\right)$ we have

$$
\mathcal{R}_{N}\left(\Delta, b, w^{\prime}, f^{\prime \prime}, c\right)_{s}=\mathcal{R}_{N}\left(\Delta, b, w^{\prime}, f^{\prime}, c\right)_{s} \prod_{j=1}^{n} \frac{\left(w_{1}^{\prime}\right)^{-1}}{1-w_{0}^{\prime} \zeta^{i-k+j}}
$$

up to multiplication by $N$-th roots of unity, where $i$ and $k$ are as in (2-12). For each 2 -simplex met by $l$ we can apply (6-4) to the corresponding tetrahedron, or the similar formula (deduced from Corollary 5.6 of [4]) for any other branching. This defines the function $\Lambda_{N}\left(\mathcal{T}^{\prime \prime}\right)$, so that we get

$$
\begin{aligned}
\mathcal{H}_{N}\left(\mathcal{T}^{\prime \prime}\right) & =\sum_{s} \prod_{\Delta \subset T} \mathcal{R}_{N}\left(\Delta, b, w^{\prime}, f^{\prime \prime}, c\right)_{s} \\
& =\sum_{s} \prod_{\Delta \subset T} \mathcal{R}_{N}\left(\Delta, b, w^{\prime}, f^{\prime}, c\right)_{s} \Lambda_{N}\left(\mathcal{T}^{\prime \prime}\right) .
\end{aligned}
$$

The conclusion follows from the equality $H_{N}(W, L, \rho)=\mathcal{H}_{N}\left(\mathcal{T}^{\prime \prime}\right)$.

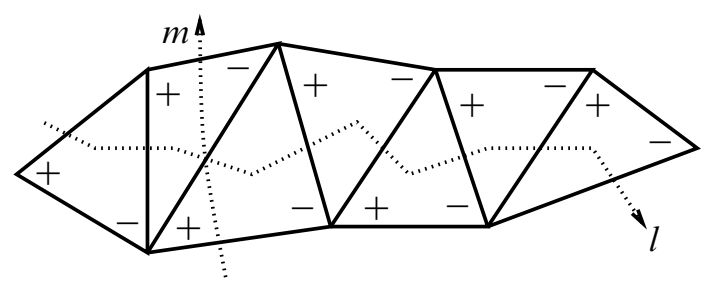

Figure 16: Flat/charge corrections for a Dehn filling

Proof of Theorem 6.7 Again for simplicity, assume that $L$ is a knot (ie it has one component). We apply the very same arguments as for the first claim of Theorem 6.4. In particular, Lemma 6.11 applies verbatim. Since $\rho(l)$ is trivial, for an arbitrary flattening the weight along $l$ (computed in the flattened $\mathcal{I}$-cusps) lies in $\mathbb{Z} \pi \sqrt{-1}$. Hence we can again use Theorem 2.20 to deduce the existence of flattenings with zero weight along $l$. Then we give the same log-branches on the 3-simplices of the singular 3-ball $C D$, in a pair above and below the disk $D$ [24, p 454]. Note that if we use $\mathcal{D}$ triangulations leading to $\mathcal{H}_{N}$ partition functions, then both parallel components $L, L^{\prime}$ that make $\bar{\lambda}$ survive in the Hamiltonian subcomplex. If we can deal with $\mathcal{H}_{N}^{e}$-ones, only the framing longitude $L^{\prime}$ survives. Hence we eventually get a distinguished flat/charged $\mathcal{I}$-triangulation for $\left(W(l), l^{*} \cup \bar{\lambda}, \rho\right)$, or $\left(W(l), l^{*} \cup \lambda^{\prime}, \rho\right)$ respectively. 


\subsection{Manifolds that fiber over $S^{1}$}

Lemma 5.11 (3) gives a practical recipe to compute the QHFT partition functions of mapping tori. A specific class of distinguished flat/charged $\mathcal{I}$-triangulations of $\left(\widetilde{W}_{[\psi]}, \widetilde{L}_{[\psi]}, \rho\right)$ is obtained by composing one for the trivial mapping cylinder $F \times[-1,1]$, say $\mathcal{T}_{\text {triv }}$, with the monodromy action on the $e$-triangulation $(T, b)$ of $F \times\{1\}$, and then gluing the two boundary components. The monodromy action can always be decomposed as a sequence of flip moves: a single flip on the ideal triangulation $T^{\prime}$ associated to $T$ defines a flip on $(T, b)$ if it is not adjacent to a marked corner, and it lifts to sequences as in Figure 17 otherwise. We view these sequences as the result of gluing tetrahedra. Hence the monodromy action determines a branched triangulated pseudomanifold $T_{S}$. This can be completed with global charges, and, as for any $\rho$ we are free to choose the cocycle in $\mathcal{T}_{\text {triv }}$, we can also complete $T_{s}$ to a flattened $\mathcal{I}$-triangulation. Equivalently we can define a sequence

$$
s:(T, b, \mathcal{L}) \rightarrow \ldots \rightarrow \psi(T, b, \mathcal{L})
$$

of $e$-triangulations with $(+)-\log -\mathcal{I}$-parameters compatible with $\rho$. Note that the edges of the associated pattern $\mathcal{T}_{s}$ of flat/charged $\mathcal{I}$-tetrahedra are disjoint from the Hamiltonian link $H$. Since $\mathcal{H}_{N}\left(\mathcal{T}_{\text {triv }}\right)={ }_{N}$ id, we deduce that $\mathcal{H}_{N}\left(\widetilde{W}_{[\psi]}, \widetilde{L}_{[\psi]}, \rho\right)={ }_{N}$ $\mathcal{H}_{N}\left(\mathcal{T}_{s}\right)$.

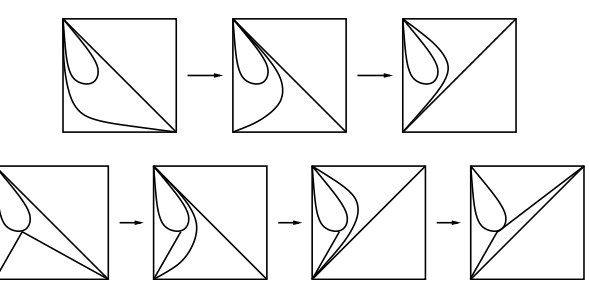

Figure 17: Lifts to $e$-triangulations (economic ones - see Remark 3.13 at the first row) of flip moves on the corresponding ideal triangulations near marked corners. The tetrahedron associated to the first flip (first and third ones for the second row) degenerates for a sequence $s$ with (-) $-\log -\mathcal{I}_{-}$ parameters.

Using a similar construction we now prove the relationship with the quantum hyperbolic invariants $H_{N}$ of fibered cusped manifolds [4]. Recall that $W \backslash L$ is homeomorphic to $\operatorname{Int}\left(\widetilde{W}_{[\psi]}\right)$. Denote by $l$ the number of components of $L$.

Proposition 6.12 If $\operatorname{Int}\left(\widetilde{W}_{[\psi]}\right)$ supports a (necessarily unique) complete hyperbolic structure with holonomy $\rho_{c}$, then $\mathcal{H}_{N}\left(\widetilde{W}_{[\psi]}, \widetilde{L}_{[\psi]}, \rho_{c}, 0,0\right)={ }_{N} N^{2 l} H_{N}(W \backslash L)$. 
Proof Let $S \backslash V$ be the fiber of $W \backslash L \rightarrow S^{1}$, equipped with an ideal triangulation $T^{\prime}$. First we show the existence of sequences $s: T^{\prime} \rightarrow \ldots \rightarrow \psi\left(T^{\prime}\right)$ of flip moves decomposing the monodromy action, such that the associated pseudomanifolds $T_{s}^{\prime}$ are topological ideal triangulations of $W \backslash L$, which moreover have maximal volume.

The first condition follows from the fact that the monodromy is homotopically aperiodic (ie pseudo-Anosov), so that $T_{s}^{\prime}$ is genuinely three-dimensional. When $S \backslash V$ is a oncepunctured torus, the second condition is a consequence of a result of Lackenby [22], showing that the monodromy ideal triangulation of Floyd and Hatcher [15] is isotopic to the canonical Epstein-Penner cellulation. More in general, since no edge of $T_{s}^{\prime}$ is homotopically trivial, the results of Francaviglia [16] imply that we can straighten the tetrahedra to oriented geodesic ones, possibly with overlappings, so that the algebraic sum of volumes is $\operatorname{Vol}(W \backslash L)$. This is known to be maximal [17].

As in Section 6.2 we can complete $T_{s}^{\prime}$ to a flat/charged $\mathcal{I}$-triangulation $\mathcal{T}^{\prime}$. Hence the invariants $H_{N}(W \backslash L)$ can be computed as trace tensors $\mathcal{H}_{N}\left(\mathcal{T}^{\prime}\right)$. We note that in the case when there are several fibrations of $W \backslash L$, or $T_{s}^{\prime}$ is not canonical, the invariance follows from Theorem 6.8 (2) in [4], which shows that any two flat/charged $\mathcal{I}$-triangulations of $W \backslash L$ with maximal volume are QHG-isomorphic.

Let us denote $\mathcal{T}_{s}^{\prime}$ the result of cutting $\mathcal{T}^{\prime}$ along the fiber. The two boundary copies are marked pleated hyperbolic surfaces $\left(T^{\prime}, b^{\prime}, \mathcal{L}\right) \rightarrow S \backslash V$ and $\left(T^{\prime}, b^{\prime}, \mathcal{L}\right) \rightarrow \psi(S \backslash V)$, with shear-bend coordinates (ie $(-)-\log -\mathcal{I}$-parameters) $\mathcal{L}$ that determine completely the log-branches of $\mathcal{T}_{s}^{\prime}$. Recall from Section 5.5 the families of flat/charged $\mathcal{I}_{-}$ triangulations $\mathcal{F}_{t}$ and $\mathcal{F}$, and let $C \in \mathcal{F}, C_{t} \in \mathcal{F}_{t}$ have boundary structures $(T, b, \mathcal{L})$, associated to $\left(T^{\prime}, b^{\prime}, \mathcal{L}\right)$, and $\left(T, b, \mathcal{L}_{t}\right)$, respectively, at $F \times\{1\}$. We have:

$$
\begin{aligned}
\mathcal{H}_{N}\left(\mathcal{T}^{\prime}\right) & =\operatorname{Tr}\left(\mathcal{H}_{N}\left(\mathcal{T}_{s}^{\prime}\right)\right) \\
& =N^{-2 l} \operatorname{Tr}\left(\mathcal{H}_{N}\left(\mathcal{T}_{s}^{\prime}\right) \otimes \mathrm{id}^{\otimes 2 l}\right) \\
& =N^{-2 l} \operatorname{Tr}\left(\mathcal{H}_{N}(C) \circ\left(\mathcal{H}_{N}\left(\mathcal{T}_{s}^{\prime}\right) \otimes \mathrm{id}^{\otimes 2 l}\right) \circ \mathcal{H}_{N}(\psi(C))^{-1}\right) \\
& =N^{-2 l} \lim _{t \rightarrow \mathrm{id}} \operatorname{Tr}\left(\mathcal{H}_{N}\left(C_{t}\right) \circ\left(\mathcal{H}_{N}\left(\mathcal{T}_{s, t}^{\prime}\right) \otimes \mathrm{id}^{\otimes 2 l}\right) \circ \mathcal{H}_{N}\left(\psi\left(C_{t}\right)\right)^{-1}\right) \\
& =N^{-2 l} \lim _{t \rightarrow \mathrm{id}} \operatorname{Tr}\left(\mathcal{H}_{N}\left(C_{t} * \mathcal{T}_{s, t}^{\prime} *\left(-\psi\left(C_{t}\right)\right)\right)\right) .
\end{aligned}
$$

Here we use the invertibility of $\mathcal{H}_{N}(C)$ (Proposition 5.13) and the equality $\mathcal{H}_{N}(C)={ }_{N}$ $\mathcal{H}_{N}(\psi(C))$. We define $\mathcal{T}_{s, t}^{\prime}$ as the continuous deformation of $\mathcal{T}_{s}^{\prime}$ obtained from the sequence

$$
s_{t}:\left(T, b, \mathcal{L}_{t}\right) \rightarrow \ldots \rightarrow \psi\left(T, b, \mathcal{L}_{t}\right)
$$

similarly as in (6-5) (see also Figure 17). In the last equality, $C_{t} * \mathcal{T}_{s, t}^{\prime} *\left(-\psi\left(C_{t}\right)\right.$ ) is for any fixed $t$ a distinguished flat/charged $\mathcal{I}$-triangulation of the mapping cylinder of $\psi$. Hence $\mathcal{H}_{N}\left(C_{t} * \mathcal{T}_{s, t}^{\prime} *\left(-\psi\left(C_{t}\right)\right)\right)$ does not depend on $t$ up to conjugacy, and we conclude with Lemma 5.11 (3). 
By following the above computation backwards, we see more in general that for any $\operatorname{PSL}(2, \mathbb{C})$-character $\rho$ that can be realized by $(-)$-Log- $\mathcal{I}$-parameters on some ideal triangulation of the fiber $S \backslash V$, we have

$$
\mathcal{H}_{N}\left(\widetilde{W}_{[\psi]}, \tilde{L}_{[\psi]}, \rho, h, k\right)=N^{2 l} \operatorname{Tr}\left(\mathcal{H}_{N}\left(\mathcal{T}_{s}^{\prime}\right)\right)
$$

where $\mathcal{T}_{s}^{\prime}$ is a pattern of flat/charged $\mathcal{I}$-tetrahedra associated to a sequence similar to (6-5), but with $e$-triangulations equipped with $(-)-\log -\mathcal{I}$-parameters compatible with $\rho$.

Remark 6.13 The formula $H_{N}(W \backslash L)=\operatorname{Tr}\left(\mathcal{H}_{N}\left(\mathcal{T}_{s}^{\prime}\right)\right)$ expresses the quantum hyperbolic invariants of fibered cusped manifolds as amplitudes between two markings of the fiber, identified with a pleated hyperbolic surface. For a similar construction based on representations of quantum Teichmüller spaces, see Bonahon and Liu [9].

\subsection{Example: the figure-eight knot complement}

Here we compute the QHFT partition functions of $\left(S^{3}, K_{0}\right)$ for $N>1$, where $K_{0}$ is the 0 -framed figure-eight knot in $S^{3}$. Recall that $S^{3} \backslash K$ is fibered over $S^{1}$, with fiber the once-punctured torus $\Sigma_{1,1}$; the 0 -framing of $K$ is induced by the fibration. For simplicity, below we consider only characters $\rho$ of injective representations $\pi_{1}\left(S^{3} \backslash K\right) \rightarrow P S L(2, \mathbb{C})$. The restriction to $\Sigma_{1,1}$ of such representations can be realized by $(-)-\log -\mathcal{I}$-parameters on any ideal triangulation of $\Sigma_{1,1}$, so that, by (6-6), we can determine the corresponding subspace (still denoted $\operatorname{Def}\left(S^{3}, K\right)$ ) of the phase space of Definition 5.9 by using the monodromy ideal triangulation.

The monodromy $\Phi: \Sigma_{1,1} \rightarrow \Sigma_{1,1}$ of $S^{3} \backslash K$ is isotopic to the hyperbolic element

$$
\left(\begin{array}{ll}
2 & 1 \\
1 & 1
\end{array}\right)=\left(\begin{array}{ll}
1 & 1 \\
0 & 1
\end{array}\right)\left(\begin{array}{ll}
1 & 0 \\
1 & 1
\end{array}\right) \quad \in S L(2, \mathbb{Z})
$$

This description of $[\Phi] \in \operatorname{Mod}\left(\Sigma_{1,1}\right)$ can be understood in terms of the Diagram of $P S L(2, \mathbb{Z})$ (see eg Floyd and Hatcher [15]) via the action of $\Phi$ on topological ideal triangulations of $\Sigma_{1,1}$, which can be represented by two flip moves. See Figure 18, where the left picture is a lift to $\mathbb{R}^{2} \backslash \mathbb{Z}^{2}$ of such a triangulation.

The monodromy ideal triangulation $T$ of $S^{3} \backslash K$ is obtained by realizing each flip move via the gluing of an ideal tetrahedron, first on a fixed triangulation of $\Sigma_{1,1}$, then on the resulting one. The remaining four free faces are identified under $\Phi$. It is not difficult to see that $T$ is isotopic to the canonical geodesic ideal triangulation of $S^{3} \backslash K$ with its complete hyperbolic structure. The gluing pattern of the tetrahedra in $T$ is shown in Figure 19.

Algebraic ${ }^{3} \mathcal{G}$ Geometric Topology, Volume 7 (2007) 


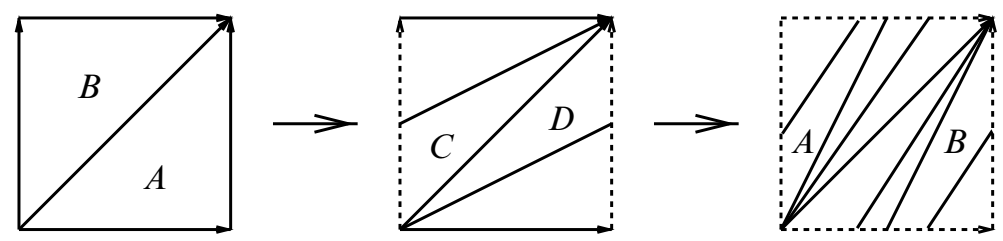

Figure 18: The composition of flip transformations for the monodromy of $S^{3} \backslash K$
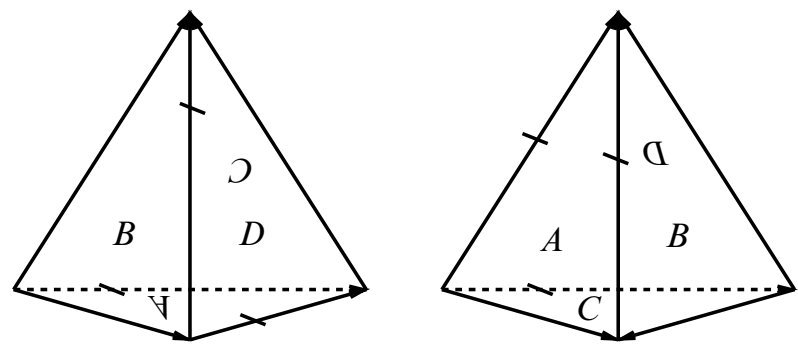

Figure 19: The face and edge identifications for the canonical geodesic ideal triangulation of $S^{3} \backslash K$

It is well-known (see [30]) that the deformation space of smooth (not necessarily complete) hyperbolic structures on $S^{3} \backslash K$ for which $T$ is geodesic is isomorphic to the algebraic set $\operatorname{Def}_{\text {hyp }}\left(S^{3} \backslash K\right) \subset \mathbb{Q}^{2} \times \mathbb{T}^{2}$ of points $\left(w_{2}, z_{0}^{-1}\right)$ such that

$$
w_{2} \in \mathbb{H}^{2} \backslash\left\{\frac{1}{2}+\frac{t}{2} i \mid t \geq \sqrt{15}\right\}, \quad z_{0}^{-1}=\frac{1}{2}+\left(\frac{1}{4}+\frac{1}{w_{2}\left(w_{2}-1\right)}\right)^{\frac{1}{2}} .
$$

In fact we can realize $w_{2}$ as a cross-ratio modulus of the edge $e_{2}$ in the tetrahedron $\Delta^{+}$with positive branching orientation (back edge in the left tetrahedron of Figure 19), and similarly for $z_{0}$ in $\Delta^{-}$. Note the exponent -1 in the formula for $z_{0}$, which is due $\mathrm{t}$ the negative branching orientation of $\Delta^{-}$. Hence $\operatorname{Def}_{\text {hyp }}\left(S^{3} \backslash K\right)$ is a subspace of

$$
\mathcal{C}=\left\{\left(w_{2}, z_{0}\right) \in(\mathbb{C} \backslash\{0,1\})^{2} \mid w_{1} w_{2}^{2} z_{0}^{-2} z_{1}^{-1}=1\right\},
$$

the whole set of solutions of the edge compatibility relations for cross-ratio moduli (see Definition 2.10). By an easy computation we find that the edge compatibility relations for log-branches and charges are:

$$
(S)\left\{\begin{array}{c}
f_{1}^{-}+2 f_{0}^{-}+2 f_{0}^{+}+f_{1}^{+} \\
\quad=\left(\arg \left(w_{1}\right)+2 \arg \left(w_{0}\right)+\arg \left(z_{1}\right)+2 \arg \left(z_{0}\right)\right) / \pi i \\
c_{1}^{-}+2 c_{0}^{-}-2 c_{0}^{+}-c_{1}^{+}=0
\end{array}\right.
$$


where the flattenings $f_{i}^{+}$and $f_{i}^{-}$correspond to the cross-ratio moduli $w_{i}$ and $z_{i}$ of $\Delta^{+}$and $\Delta^{-}$, respectively. Hence, by (2-2) we see that the QHFT phase space $\operatorname{Def}\left(S^{3}, K\right)$ is the covering of $\mathcal{C}$ given by

$$
\begin{array}{r}
\operatorname{Def}\left(S^{3}, K\right)=\left\{\left(\left(w_{2} ; f_{2}^{+}-c_{2}^{+}, f_{0}^{+}-c_{0}^{+}\right),\left(z_{0} ; f_{0}^{-}+c_{0}^{-}, f_{1}^{-}+c_{1}^{-}\right)\right) \in \hat{\mathbb{C}} \times \widehat{\mathbb{C}}\right. \\
\left.\mid\left(w_{2}, z_{0}\right) \in \mathcal{C},(S) \text { is satisfied }\right\} .
\end{array}
$$

Fixing the dilation factors $\mu(m)$ and $\mu(l)$ of the standard meridian $m$ and longitude $l$ of $K$, we get geometrically meaningful subspaces of $\operatorname{Def}\left(S^{3}, K\right)$. Below we follow the orientation conventions and some computations in [24, Section 15] which we reproduce for clarity. First we have

$$
\mu(m)=z_{2} w_{2} \quad, \quad \mu(l)=w_{0}^{2} w_{2}^{-2} .
$$

Consider the complete hyperbolic structure of $S^{3} \backslash K$. It is obtained by solving $\mu(m)=$ 1 or $\mu(l)=1$ in $\operatorname{Def}_{\text {hyp }}\left(S^{3} \backslash K\right)$, the solution being $z_{j}=\left(w_{j}\right)^{*}=\exp (\pi \sqrt{-1} / 3)$. The possible weights $k \in H^{1}\left(\partial\left(S^{3} \backslash U(K)\right)\right.$; $\left.\mathbb{C}\right)$ for this solution actually take values in $\pi \sqrt{-1} \mathbb{Z}$ (see Neumann [24, Corollary 5.4]). Corresponding flattenings satisfy

$$
f_{2}^{-}+f_{2}^{+}=k(m) / \pi \sqrt{-1} \quad, \quad 2 f_{0}^{+}-2 f_{2}^{+}=k(l) / \pi \sqrt{-1}
$$

which we rewrite as

(6-9) $f_{0}^{-}+f_{0}^{+}+f_{1}^{-}+f_{1}^{+}=-k(m) / \pi \sqrt{-1}$ and $4 f_{0}^{+}+2 f_{1}^{+}+2=k(l) / \pi \sqrt{-1}$

by using $f_{0}^{ \pm}+f_{1}^{ \pm}+f_{2}^{ \pm}=\mp 1$. Now (6-8) simplifies to

$$
f_{1}^{-}+2 f_{0}^{-}+2 f_{0}^{+}+f_{1}^{+}=0 .
$$

Together with (6-9) this gives

$$
f_{0}^{-}=\frac{k(m)}{\pi \sqrt{-1}}-f_{0}^{+}, \quad\left\{\begin{array}{l}
f_{1}^{+}=-2 f_{0}^{+}+\frac{1}{2}\left(\frac{k(l)}{\pi \sqrt{-1}}-2\right) \\
f_{1}^{-}=+2 f_{0}^{+}-2 \frac{k(m)}{\pi \sqrt{-1}}-\frac{1}{2}\left(\frac{k(l)}{\pi \sqrt{-1}}-2\right) .
\end{array}\right.
$$

Here the first equation comes directly from (6-10) and the first identity in (6-9). The third follows from it and and

$$
f_{1}^{-}+2 f_{0}^{-}=-\left(f_{1}^{+}+2 f_{0}^{+}\right)=-\frac{1}{2}\left(\frac{k(l)}{\pi \sqrt{-1}}-2\right) .
$$

The second equation of (6-11) is a straightforward consequence of the others. Finally, the weight $h \in H^{1}\left(S^{3} \backslash K ; \mathbb{Z} / 2 \mathbb{Z}\right)$ is 0 when and only when $k(m) \in 2 \sqrt{-1} \pi \mathbb{Z}$. Also, since $T$ is quasi-geometric we have $*_{b} *_{w}=1$, so that integral charges are obtained by letting $c_{i}^{+}=-f_{i}^{+}$on $\Delta^{+}$and $c_{i}^{-}=+f_{i}^{-}$on $\Delta^{-}$. 
Clearly (6-11) still holds true for points of $\operatorname{Def}\left(S^{3}, K\right)$ sufficiently near the complete solution, if now $k(m) / \pi \sqrt{-1}$ and $k(l) / \pi \sqrt{-1}$ are replaced with $\tilde{k}(m)=(k(m)-$ $\log (\mu(m))) / \sqrt{-1} \pi$ and $\tilde{k}(l)=(k(l)-\log (\mu(l))) / \sqrt{-1} \pi$. Hence, in the vicinity of the complete structure we have the strata

$$
\begin{aligned}
& \left\{\left(\left(w_{2} ; 2 f_{0}^{+}-\tilde{k}(l), 2 f_{0}^{+}\right),\left(z_{0} ; 2\left(\tilde{k}(m)-f_{0}^{+}\right),\right.\right.\right. \\
& \left.\left.\qquad 4\left(f_{0}^{+}-\tilde{k}(m)\right)+2-\tilde{k}(l)\right), f_{0}^{+} \in \mathbb{Z}\right\},
\end{aligned}
$$

parametrized by the lifts $k(m)$ and $k(l)$ of $\mu(m)$ and $\mu(l)$.

Let us now consider points of $\mathcal{C}$ corresponding to hyperbolic structures whose metric completion is obtained from $S^{3} \backslash K$ by $(p, q)$ Dehn filling. We have

$$
p \log (\mu(m))+q \log (\mu(l))=2 \pi \sqrt{-1} .
$$

Here we have to distinguish between the weights of flattenings and charges, which behave in a different way (see our notation before Theorem 5.3). Since for weights of flattenings we must have $p k_{f}(m)+q k_{f}(l)=0$ (the compatibility condition about the edges making the core of the added solid torus), the flattenings $f=f^{\prime \prime}$ of Lemma 6.3 satisfy

$$
p\left(f_{2}^{-}+f_{2}^{+}\right)+q\left(2 f_{0}^{+}-2 f_{2}^{+}\right)=-p\left(f_{0}^{-}+f_{0}^{+}+f_{1}^{-}+f_{1}^{+}\right)+q\left(4 f_{0}^{+}+2 f_{1}^{+}+2\right)=-2 .
$$

Let us fix $r, s \in \mathbb{Z}$ such that $p s-q r=1$. Solving simultaneously (6-10) and the last equation gives:

$$
\begin{aligned}
p\left(f_{0}^{-}+f_{0}^{+}\right)+ & 2 q\left(1-2 f_{0}^{-}-f_{1}^{-}\right)=-2 \\
& \Longleftrightarrow\left\{\begin{array}{l}
s=-\frac{1}{2}\left(f_{0}^{-}+f_{0}^{+}\right) \\
r=1-2 f_{0}^{-}-f_{1}^{-}
\end{array}\right. \\
& \Longleftrightarrow\left\{\begin{array}{l}
f_{0}^{-}=-2 s-f_{0}^{+} \\
f_{1}^{-}=1-r+2 f_{0}^{+}+4 s
\end{array}\right.
\end{aligned}
$$

Finally, we get

$$
f_{1}^{+}=r-1-2 f_{0}^{+}, \quad\left\{\begin{array}{l}
f_{0}^{-}=-2 s-f_{0}^{+} \\
f_{1}^{-}=1-r+2 f_{0}^{+}+4 s .
\end{array}\right.
$$

The parity condition $f_{0}^{+}+f_{1}^{+}+f_{0}^{-}+f_{1}^{-} \in 2 \mathbb{Z}$ (for the class $h \in H^{1}\left(S^{3} \backslash K ; \mathbb{Z} / 2 \mathbb{Z}\right.$ ) to be 0 ) is automatically satisfied. Finally, we are free to choose any charge with 0 weight on the meridian curve $C=m^{p} l^{q}$ of the added solid torus. This is obtained directly from the system (6-11) by replacing the $f_{i}^{ \pm}$with $\mp f_{i}^{ \pm}$and taking the charge weight $k=k_{c}$, with the additional condition $p k_{c}(m)+q k_{c}(l)=0$. 
Now we can compute the QHFT invariants of $\left(S^{3}, K_{0}\right)$ as functions on $\operatorname{Def}\left(S^{3}, K\right)$. Given an $N$-state $s$ of $T$, put $\alpha=s(\widehat{2}), \beta=s(\widehat{0}), \gamma=s(\widehat{3})$ and $\delta=s(\widehat{1})$, where $\widehat{i}$ denotes the face of $\Delta^{+}$opposite to the $i$-th vertex. By (6-6) we have:

$$
\begin{aligned}
N^{-2} \mathcal{H}_{N}\left(S^{3}, K_{0}, \rho\right) & \\
= & \sum_{\alpha, \beta, \gamma, \delta=0}^{N-1} \mathcal{R}_{N}\left(\Delta^{+}, b^{+}, w, f^{+}, c^{+}\right)_{\gamma, \delta}^{\alpha, \beta} \mathcal{R}_{N}\left(\Delta^{-}, b^{-}, z, f^{-}, c^{-}\right)_{\beta, \alpha}^{\delta, \gamma} \\
= & \sum_{\alpha, \beta, \gamma, \delta=0}^{N-1}\left(w_{0}^{\prime-c_{1}^{+}} w_{1}^{\prime c_{0}^{+}}\right)^{\frac{N-1}{2}} \frac{g\left(w_{0}^{\prime}\right)}{g(1)} \zeta^{\gamma \beta+(m+1) \gamma^{2}} \\
& \times\left(z_{0}^{\prime-c_{1}^{-}} z_{1}^{\prime c_{0}^{-}}\right)^{\frac{N-1}{2}} \frac{\left[z_{0}^{\prime}\right] g(1)}{g\left(z_{0}^{\prime}\right)} \zeta^{-\alpha \delta-(m+1) \delta^{2}} \frac{\delta(\alpha+\beta-\gamma)}{\omega\left(z_{0}^{\prime} / \zeta, z_{1}^{\prime-1} \mid \beta-\delta\right)} \\
= & \left.\frac{\left[z_{0}^{\prime}\right] g\left(w_{0}^{\prime}\right)}{g\left(z_{0}^{\prime}\right)}\left(w_{0}^{\prime-c_{1}^{+}} w_{1}^{\prime c_{0}^{+}} z_{0}^{\prime-c_{1}^{-}} z_{1}^{\prime c}\right)^{-}\right)^{\frac{N-1}{2}} \\
& \times \sum_{\alpha, \beta=0}^{N-1} \zeta^{\beta^{2}-\alpha^{2}} \omega\left(w_{0}^{\prime}, w_{1}^{\prime-1} \mid N-\beta\right) \omega\left(z_{0}^{\prime} / \zeta, z_{1}^{\prime-1} \mid N-\alpha\right)^{-1} .
\end{aligned}
$$

By the proof of Proposition 8.6 in [4] we have

$$
\frac{\left[z_{0}^{\prime}\right] g\left(w_{0}^{\prime}\right)}{g\left(z_{0}^{\prime}\right)}=\frac{g\left(\left(z_{0}^{\prime}\right)^{*}\right)^{*} g\left(w_{0}^{\prime}\right)}{|g(1)|^{2}}
$$

and

$$
\omega\left(z_{0}^{\prime} / \zeta, z_{1}^{\prime-1} \mid N-\alpha\right)^{-1}=\omega\left(\left(z_{0}^{\prime}\right)^{*},\left(z_{1}^{\prime-1}\right)^{*} \mid \alpha\right)^{*}
$$

where $z^{*}$ is the complex conjugate of $z$. Thus, setting

$$
S\left(w_{0}^{\prime}, w_{1}^{\prime}\right)=\sum_{\beta=0}^{N-1} \zeta^{\beta^{2}} \omega\left(w_{0}^{\prime}, w_{1}^{\prime-1} \mid \beta\right)=1+\sum_{\beta=1}^{N-1} \zeta^{\beta^{2}} \prod_{k=1}^{\beta} \frac{w_{1}^{\prime-1}}{1-w_{0}^{\prime} \zeta^{k}}
$$

we get

$$
\begin{aligned}
& \mathcal{H}_{N}\left(S^{3}, K_{0}, \rho\right) \\
& \quad=N^{2}\left(w_{0}^{\prime-c_{1}^{+}} w_{1}^{\prime c_{0}^{+}} z_{0}^{\prime-c_{1}^{-}} z_{1}^{\prime c_{0}^{-}}\right)^{\frac{N-1}{2}} \frac{g\left(\left(z_{0}^{\prime}\right)^{*}\right)^{*} g\left(w_{0}^{\prime}\right)}{|g(1)|^{2}} S\left(w_{0}^{\prime}, w_{1}^{\prime}\right) S\left(\left(z_{0}^{\prime}\right)^{*},\left(z_{1}^{\prime}\right)^{*}\right)^{*} .
\end{aligned}
$$

For instance, by using (6-11) with $k(m)=k(l)=f_{0}^{+}=0$ and the global charge with $c_{i}^{ \pm}=\mp f_{i}^{ \pm}$, we see that for the complete hyperbolic structure $\rho_{\text {comp }}$ on $S^{3} \backslash K$ we 
have

$$
z_{0}^{\prime}=\left(w_{0}^{\prime}\right)^{*}=\exp (-i \pi / 3 N) \quad, \quad z_{1}^{\prime}=\left(w_{1}^{\prime}\right)^{*}=\exp (5 i \pi / 3 N)
$$

Hence

$$
\mathcal{H}_{N}\left(S^{3}, K_{0}, \rho_{\text {comp }}\right)=N^{2} \frac{\left|g\left(e^{i \pi / 3 N}\right)\right|^{2}}{|g(1)|^{2}}\left|S\left(e^{i \pi / 3 N}, e^{-5 i \pi / 3 N}\right)\right|^{2} \in \mathbb{R}_{>0} .
$$

Let us finally consider hyperbolic $(p, q)$ Dehn filling of $S^{3} \backslash K$. Denote $S^{3}\left(K_{(p, q)}\right)$ the surgered manifold, $L$ the core of the surgery, and $\rho_{(p, q)}$ its hyperbolic holonomy. Because of (6-11) and (6-12) the difference $f^{\prime \prime}-f$ is given on the edges $e_{0}, e_{1}$ and $e_{2}$ of $\Delta^{+}$(resp. $\Delta^{-}$) by $0, r$ and $-r$ (resp. $-2 s,-r+4 s$ and $r-2 s$ ). Put $N=2 m+1$. From Theorem 6.4 we deduce

$$
\begin{aligned}
\mathcal{H}_{N}\left(S^{3}\left(K_{(p, q)}\right)\right. & , L, \rho(p, q))= \\
N^{2} & \left(w_{0}^{\prime-c_{1}^{+}} w_{1}^{\prime c_{0}^{+}} z_{0}^{\prime-c_{1}^{-}} z_{1}^{\prime c_{0}^{-}}\right)^{\frac{N-1}{2}} \frac{g\left(\left(z_{0}^{\prime}\right)^{*}\right)^{*} g\left(w_{0}^{\prime}\right)}{|g(1)|^{2}} \\
& \times \sum_{\alpha, \beta=0}^{N-1} \zeta^{\beta^{2}-\alpha^{2}+r \beta(m+1)} \times \prod_{j=1}^{-2 s} \frac{z_{1}^{\prime-1}}{1-z_{0}^{\prime} \zeta^{-(j+\alpha)}} \omega\left(\left(z_{0}^{\prime}\right)^{*},\left(z_{1}^{\prime-1}\right)^{*} \mid \alpha\right)^{*} .
\end{aligned}
$$

Remark 6.14 Denote by $\mathcal{H}_{N}^{0}(\rho)$ the subsum in $\mathcal{H}_{N}\left(S^{3}, K_{0}, \rho\right)$ made of the summands with no $\zeta$ phase factor:

$$
\begin{aligned}
\mathcal{H}_{N}^{0}(\rho)=\left(w_{0}^{\prime-c_{1}^{+}} w_{1}^{\prime c_{0}^{+}} z_{0}^{\prime-c_{1}^{-}} z_{1}^{\prime c_{0}^{-}}\right)^{\frac{N-1}{2}} \frac{g\left(\left(z_{0}^{\prime}\right)^{*}\right)^{*} g\left(w_{0}^{\prime}\right)}{|g(1)|^{2}} & \\
& \times \sum_{\alpha=0}^{N-1} \prod_{k=1}^{\alpha}\left(\frac{w_{1}^{\prime-1}}{1-w_{0}^{\prime} \zeta^{k}}\right)\left(\frac{z_{1}^{\prime-1}}{1-z_{0}^{\prime} \zeta^{-k}}\right) .
\end{aligned}
$$

At the complete structure we have positivity of the summands ( $w_{0}^{\prime}$ being, for example, as in (6-13)):

$$
\mathcal{H}_{N}^{0}\left(\rho_{\text {comp }}\right)=\frac{\left|g\left(w_{0}^{\prime}\right)\right|^{2}}{|g(1)|^{2}} \sum_{\beta=0}^{N-1} \prod_{k=1}^{\beta} \frac{1}{\left|1-w_{0}^{\prime} \zeta^{k}\right|^{2}} .
$$

This can be compared to the Kashaev's invariant $\langle K\rangle_{N}$ of $K$ (ie the Jones polynomial $J_{N}(K)$ associated to the $N$-th dimensional irreducible representation of $s l(2, \mathbb{C})$, 
normalized as 1 on the unknot and evaluated at $\zeta$ ). Indeed we have

$$
\langle K\rangle_{N}=\left.\left(\frac{J_{N}(K)}{J_{N}(O)}\right)\right|_{\zeta}=\sum_{\beta=0}^{N-1} \prod_{i=1}^{\beta}\left|1-\zeta^{i}\right|^{2}=N^{2} \sum_{\beta=0}^{N-1} \prod_{k=1}^{\beta} \frac{1}{\left|1-\zeta^{k}\right|^{2}}
$$

where $\prod_{i=1}^{N-1}\left(1-\zeta^{i}\right)=N$ is used in the second equality. It is known that the ratio $\left(J_{N}(K) / J_{N}(O)\right)_{\mid \zeta}$ essentially coincides with the quantum hyperbolic invariant $H_{N}\left(S^{3}, K, \rho_{0}\right)$, where $\rho_{0}$ is the (necessary trivial) flat $\operatorname{PSL}(2, \mathbb{C})$-character of $S^{3}$ (see eg [3, Section 5]). What we see here has a different flavor: a relationship between $\langle K\rangle_{N}$ and the function $\mathcal{H}_{N}\left(S^{3}, K_{0}, \rho\right)$ on $\operatorname{Def}\left(S^{3}, K\right)$.

Remark 6.15 Recall the space $\mathcal{C}$ in (6-7). As already mentioned after Definition 2.10, we have a holonomy map hol: $\mathcal{C} \rightarrow X$ to the character variety $X=X\left(\pi_{1}\left(S^{3} \backslash K\right)\right)$. (See Riley [28] or González-Acuña and Montesinos-Amilibia [18] for a complete description of the latter). The map hol is generically $2: 1$ and the image contains the geometric component of $X$ [11]. We can express the above partition functions in terms of standard generators of $X$ by the following observation. Considering $S^{3} \backslash K$ as the mapping torus of the monodromy $\Phi$, the edges $e_{0}, e_{1}$ of $\Delta^{+}$are identified with a longitude $l$ and meridian $m$ of the punctured torus $\Sigma_{1,1}$, and in $\Delta^{-}$we have $\left(e_{0}^{\prime}\right.$ is opposite to $\left.e_{0}\right)$ :

$$
e_{0}=\Phi(l . m), \quad e_{1}=\Phi(l), \quad e_{0}^{\prime}=\Phi(m) .
$$

As above, assume that $\rho$ has nontrivial holonomy at $m, l$ and $l . m$. Take a flat/charged $\mathcal{I}$-triangulation of $\left(S^{3}, K_{0}, \rho\right)$ as in Section 6.3, with $P S L(2, \mathbb{C})$-valued cocycle $z$. Denote $z_{l}$ the value at $l$, and so on. Note that $z_{\Phi(m)}=A z_{m} A^{-1}$, where $A=z\left(S^{1}\right)$, the cocycle value on the standard meridian of the knot $K$. Then the cross ratio moduli of $\Delta^{+}$and $\Delta^{-}$are given by

$$
\begin{aligned}
& w_{0}=\left[0: z_{l}(0): z_{l} z_{m}(0): z_{\Phi(m)}(0)\right] \\
& z_{0}=\left[0: z_{\Phi(l)} z_{\Phi(m)}(0): z_{\Phi(l)} z_{\Phi(m)} z_{\Phi(l)}(0): z_{m} z_{l}(0)\right] .
\end{aligned}
$$

We use the branching to remove the two-fold ambiguity of hol, as it allows to specify an equivariant association of a fixed point for each peripheral subgroup of $\rho\left(\pi_{1}\left(S^{3} \backslash K\right)\right)$.

Remark 6.16 Formulas for $\mathcal{H}_{1}\left(S^{3}, K_{0}, \rho\right)$, the Cheeger-Chern-Simons invariants of $\operatorname{PSL}(2, \mathbb{C})$-characters of $S^{3} \backslash K$ with fixed weights, come exactly in the same way. In the peculiar situation of the complete hyperbolic structure with the weight 0 and its hyperbolic Dehn fillings, they coincide with those of [24, Section 15]. For more general characters the formulas take the same form, but now the arguments live in the space obtained from $\operatorname{Def}\left(S^{3}, K\right)$ by forgetting the charges. 


\section{References}

[1] W Abikoff, The real analytic theory of Teichmüller space, Lecture Notes in Mathematics 820, Springer, Berlin (1980) MR590044

[2] M Atiyah, Topological quantum field theories, Inst. Hautes Études Sci. Publ. Math. (1988) 175-186 (1989) MR1001453

[3] S Baseilhac, R Benedetti, Quantum hyperbolic invariants of 3-manifolds with PSL(2, C)-characters, Topology 43 (2004) 1373-1423 MR2081430

[4] S Baseilhac, R Benedetti, Classical and quantum dilogarithmic invariants of flat PSL(2, C) -bundles over 3-manifolds, Geom. Topol. 9 (2005) 493-569 MR2140989

[5] S Baseilhac, R Benedetti, Teichmüller space and cylinders in quantum hyperbolic field theories, from: "Handbook on Teichmüller Theory", (A Papadopoulos, editor), volume II, EMS Publishing House, Zürich (2008)

[6] R Benedetti, F Bonsante, Canonical Wick rotations in 3-dimensional gravity, to appear in Mem. Amer. Math. Soc. arXiv:math.DG/0508485

[7] R Benedetti, C Petronio, Lectures on hyperbolic geometry, Universitext, Springer, Berlin (1992) MR1219310

[8] F Bonahon, Shearing hyperbolic surfaces, bending pleated surfaces and Thurston's symplectic form, Ann. Fac. Sci. Toulouse Math. (6) 5 (1996) 233-297 MR1413855

[9] F Bonahon, X Liu, Representations of the quantum Teichmuller space and invariants of surface diffeomorphisms arXiv:math.GT/0407086

[10] R D Canary, D B A Epstein, P Green, Notes on notes of Thurston, from: “Analytical and geometric aspects of hyperbolic space (Coventry/Durham, 1984)", London Math. Soc. Lecture Note Ser. 111, Cambridge Univ. Press, Cambridge (1987) 3-92 MR903850

[11] A Champanerkar, A-polynomial and Bloch invariants of hyperbolic 3-manifolds, $\mathrm{PhD}$ thesis, Columbia University (2003)

[12] Y-E Choi, Positively oriented ideal triangulations on hyperbolic three-manifolds, Topology 43 (2004) 1345-1371 MR2081429

[13] J L Dupont, C K Zickert, A dilogarithmic formula for the Cheeger-Chern-Simons class, Geom. Topol. 10 (2006) 1347-1372 MR2255500

[14] D B A Epstein, A Marden, Convex hulls in hyperbolic space, a theorem of Sullivan, and measured pleated surfaces, from: "Analytical and geometric aspects of hyperbolic space (Coventry/Durham, 1984)", London Math. Soc. Lecture Note Ser. 111, Cambridge Univ. Press, Cambridge (1987) 113-253 MR903852

[15] W Floyd, A Hatcher, Incompressible surfaces in punctured-torus bundles, Topology Appl. 13 (1982) 263-282 MR651509 
[16] S Francaviglia, Algebraic and geometric solutions of hyperbolicity equations, Topology Appl. 145 (2004) 91-118 MR2100867

[17] S Francaviglia, Hyperbolic volume of representations of fundamental groups of cusped 3-manifolds, Int. Math. Res. Not. (2004) 425-459 MR2040346

[18] F González-Acuña, J M Montesinos-Amilibia, On the character variety of group representations in $\operatorname{SL}(2, \mathbb{C})$ and $\operatorname{PSL}(2, \mathbb{C})$, Math. Z. 214 (1993) 627-652 MR1248117

[19] M Heusener, J Porti, The variety of characters in PSL(2, C) arXiv: math.GT/0302075

[20] R M Kashaev, The hyperbolic volume of knots from the quantum dilogarithm, Lett. Math. Phys. 39 (1997) 269-275 MR1434238

[21] R M Kashaev, Coordinates for the moduli space of flat $\operatorname{PSL}(2, \mathbb{R})$-connections, Math. Res. Lett. 12 (2005) 23-36 MR2122727

[22] M Lackenby, The canonical decomposition of once-punctured torus bundles, Comment. Math. Helv. 78 (2003) 363-384 MR1988201

[23] W D Neumann, Combinatorics of triangulations and the Chern-Simons invariant for hyperbolic 3-manifolds, from: “Topology '90 (Columbus, OH, 1990)”, Ohio State Univ. Math. Res. Inst. Publ. 1, de Gruyter, Berlin (1992) 243-271 MR1184415

[24] W D Neumann, Extended Bloch group and the Cheeger-Chern-Simons class, Geom. Topol. 8 (2004) 413-474 MR2033484

[25] A Papadopoulos, R C Penner, La forme symplectique de Weil-Petersson et le bord de Thurston de l'espace de Teichmüller, C. R. Acad. Sci. Paris Sér. I Math. 312 (1991) 871-874 MR1108510

[26] R C Penner, The decorated Teichmüller space of punctured surfaces, Comm. Math. Phys. 113 (1987) 299-339 MR919235

[27] C Petronio, J Porti, Negatively oriented ideal triangulations and a proof of Thurston's hyperbolic Dehn filling theorem, Expo. Math. 18 (2000) 1-35 MR1751141

[28] R Riley, Nonabelian representations of 2-bridge knot groups, Quart. J. Math. Oxford Ser. (2) 35 (1984) 191-208 MR745421

[29] Y Sözen, F Bonahon, The Weil-Petersson and Thurston symplectic forms, Duke Math. J. 108 (2001) 581-597 MR1838662

[30] W P Thurston, The Geometry and Topology of Three-Manifolds, Princeton Univ. Math. Dept. Lecture Notes (1979)

[31] V G Turaev, Homotopy field theory in dimension 3 and crossed group-categories arXiv:math.GT/0005291

[32] V G Turaev, Quantum invariants of knots and 3-manifolds, de Gruyter Studies in Mathematics 18, Walter de Gruyter \& Co., Berlin (1994) MR1292673 
[33] V G Turaev, O Y Viro, State sum invariants of 3-manifolds and quantum $6 j$-symbols, Topology 31 (1992) 865-902 MR1191386

Université Grenoble I

Institut Fourier, UMR CNRS 558, 2100 rue des Maths, BP 74, F-38402 Saint Martin d'Hères Cedex, FRANCE

Dipartimento di Matematica, Università di Pisa

Largo Bruno Pontecorvo 5, I-56127 Pisa, ITALY

stephane.baseilhac@ujf-grenoble.fr, benedett@dm.unipi.it

Received: 16 November 2006 